\title{
Bott Periodicity for Fibred Cusp Operators
}

\author{
by \\ Frédéric Rochon
}

Bachelor of Science, Université de Montréal, June 2000

Master of Science, Université du Québec à Montréal, December 2001

Submitted to the Department of Mathematics in partial fulfillment of the requirements for the degree of

Doctor of Philosophy

at the

\section{MASSACHUSETTS INSTITUTE OF TECHNOLOGY}

June 2005

(c) Frédéric Rochon, MMV. All rights reserved.

The author hereby grants to MIT permission to reproduce and distribute publicly paper and electronic copies of this thesis document in whole or in part.

Author

Department of Mathematics April 29, 2005

Certified by

Richard B. Melrose Professor of Mathematics Thesis Supervisor

Accepted by

Pavel Etingof Chairman, Department Committee on Graduate Students 


\title{
Bott Periodicity for Fibred Cusp Operators \\ by \\ Frédéric Rochon
}

\author{
Submitted to the Department of Mathematics \\ on April 29, 2005, in partial fulfillment of the \\ requirements for the degree of \\ Doctor of Philosophy
}

\begin{abstract}
In the framework of fibred cusp operators on a manifold $X$ associated to a boundary fibration $\Phi: \partial X \rightarrow Y$, the homotopy groups of the space $G_{\Phi}^{-\infty}(X ; E)$ of invertible smoothing perturbations of the identity are computed in terms of the $K$-theory of $T^{*} Y$. It is shown that there is a periodicity, namely the odd and the even homotopy groups are isomorphic among themselves. To obtain this result, one of the important steps is the description of the index of a Fredholm smoothing perturbation of the identity in terms of an associated $K$-class in $K_{c}^{0}\left(T^{*} Y\right)$.

Thesis Supervisor: Richard B. Melrose

Title: Professor of Mathematics
\end{abstract}




\section{Acknowledgments}

First of all, I would like to warmly thank my advisor Richard B. Melrose. He was very generous of his time and ideas and it was a real pleasure to work with him. I would like also to thank András Vasy, who careful read my thesis and suggested many improvements. I would like also to thank François Lalonde, my master's advisor while I was in UQAM in 2000-2001, who initiated me to mathematical research and encouraged me to study abroad. I am very grateful to the NSERC and the FQRNT for their generous financial support during all of my graduate studies.

I formulated the main question my thesis is answering after a mathematical conversation I had with Benoit Charbonneau and I am very thankful to him for that. All my years in Boston were very pleasant and I would like to thank all my current and former apartmentmates for that: Nenad Dedić, Nataša Šešum, Natasha Bushueva, Kári Ragnarsson, Silvia Montarani and Gabriele Mondello. I would like to thank also my friends Étienne Rassart, Cilanne Boulet, Thomas Gervais, Tymon Barwicz, Christine Sobczak, Ariane Verdy, François Blanchette and Alex Ghitza who helped me feel good when I was missing Montréal and Canada. I would like to thank friends from the mathematics department: Morten Honsen, Bridget Turner, Zhou Zhang, Vasiliy Dolgushev, Alexei Oblomkov, Andrew Brooke-Taylor, Karen Bernhardt, Michael Ching, John Hopkinson, Damiano Testa, Daniela De Silva, Bianca Santoro and Sandra Francisco. I would like also to thank all the people involved in the international film club of MIT: Alexandru Salcianu, Onur Ozcan, Ji-Eun Kim and Cansu Tunca. I would like to thank my friends from Montréal: Sébastien Dubois, Sébastien Landry, Christian Morin and Sébastien Roch.

Enfin et surtout, Je voudrais remercier toute ma famille: Mes parents André et Julie qui m'ont toujours encouragé à poursuivre des études et m'ont toujours supporté moralement et financièrement; mon frère Étienne, qui, par les chauds après-midis d'été de la banlieue longueuilloise m'a joué de tendres mélodies à la guitare alors qu'au loin on entendait le doux ronronnement des tondeuses; ma soeur Justine, dont le nom dans les moments de solitude et de désespoir m'était gentiment murmuré par le souffle du vent; mon frère Xavier, qui malgré ses cheveux roux a su me convaincre de voter pour lui aux prochaines élections; ma soeur Valérie, qui a bien voulu cesser de grandir pour rester ma petite soeur dans tous les sens du terme. 


\section{Contents}

$\begin{array}{llr}1 & \text { Introduction } & 9\end{array}$

$2 \quad K$-theory and Bott periodicity 13

$2.1 \quad K$-theory . . . . . . . . . . . . . . . . . . 13

$2.2 \quad K$-theory and index theory . . . . . . . . . . . . . . . 19

2.3 Bott periodicity for classical pseudodifferential operators on a closed manifold ...................... 23

3 Fibred Cusp Operators 29

3.1 The framework and the definition . . . . . . . . . . . . . . 29

3.2 Some properties of fibred cusp operators . . . . . . . . . . . . 32

4 An Analytical Formula for the Index $\quad 35$

4.1 The trace-defect formula . . . . . . . . . . . . . . . 35

4.2 The index in terms of the trace-defect formula . . . . . . . . . . 38

5 The Associated $K$-class 43

5.1 Spectral Sections . . . . . . . . . . . . . . . . . 43

5.2 Construction of the $K$-Class $\ldots \ldots \ldots \ldots \ldots$

5.2 .1 The case where $\operatorname{dim} Z>0 \ldots \ldots . \ldots . \ldots 46$

5.2 .2 The case where $\operatorname{dim} Z=0 \ldots \ldots \ldots . \ldots . \ldots 52$

5.2 .3 The associated $K$-class $\ldots \ldots \ldots \ldots . \ldots \ldots$

6 The Index in terms of $K$-theory $\mathbf{5 5}$

6.1 Reduction to the case of a scattering operator . . . . . . . . . 55

6.2 Computation of the index . . . . . . . . . . . . . . 57

6.3 The Index in the case $Y=\mathbb{S}^{1} \ldots \ldots \ldots 1$

7 The Homotopy Groups of $G_{\Phi}^{-\infty} \quad \mathbf{6 5}$

7.1 Various spaces and a related Serre fibration . . . . . . . . . . . . 66

7.2 The boundary homomorphism seen as an index map . . . . . . . . . . 69

7.3 Surjectivity of the boundary homomorphism and the main theorem . 72

7.4 A related exact sequence . . . . . . . . . . . . . . . . 75

7.5 The particular case of cusp operators . . . . . . . . . . . . 76

7.6 The case $Y=\mathbb{S}^{1} \ldots \ldots \ldots \ldots$. . . . . . . . . . . . . 78 


\section{Chapter 1}

\section{Introduction}

For classical pseudodifferential operators on a closed manifold $X$ acting on some complex vector bundle $E$, Bott periodicity arises by considering the group

$$
G^{-\infty}(X ; E)=\left\{\operatorname{Id}+Q \mid Q \in \Psi^{-\infty}(X ; E), \quad \operatorname{Id}+Q \text { is invertible }\right\}
$$

of invertible smoothing perturbations of the identity. This becomes a topological group by taking the $\mathcal{C}^{\infty}$-topology induced by the identification of smoothing operators with their Schwartz kernels, which are smooth sections of some bundle over $X \times$ $X$. If $\Delta^{E}$ is any Laplacian acting on sections of $E$, and if $\left\{f_{i}\right\}_{i \in \mathbb{N}}$ is a basis of $\mathrm{L}^{2}(X ; E)$ coming from a sequence of orthonormal eigensections of $\Delta^{E}$ with increasing eigenvalues, then there is an isomorphism of topological groups

$$
\begin{aligned}
f_{\Delta^{E}}: \quad G^{-\infty}(X ; E) & \rightarrow \mathcal{G}^{-\infty} \\
\operatorname{Id}+Q & \mapsto \delta_{i j}+\left\langle f_{i}, Q f_{j}\right\rangle,
\end{aligned}
$$

where $\mathcal{G}^{-\infty}$ is the group of invertible semi-infinite matrices $\delta_{i j}+\mathcal{Q}_{i j}$ such that

$$
\|\mathcal{Q}\|_{k}=\sum_{i, j}(i+j)^{k}\left|\mathcal{Q}_{i j}\right|<\infty, \quad \forall k \in \mathbb{N}_{0}
$$

the topology of $\mathcal{G}^{-\infty}$ being the one induced by the norms $\|\cdot\|_{k}, k \in \mathbb{N}_{0}$.

This isomorphism indicates that the topology of $G^{-\infty}(X ; E)$ does not depend at all on the geometry of $X$ and $E$. Since the direct limit

$$
\mathrm{GL}(\infty, \mathbb{C})=\lim _{k \rightarrow \infty} \mathrm{GL}(k, \mathbb{C})
$$

is a weak deformation retract (see definition 7.16 below) of $\mathcal{G}^{-\infty}$, they share the same homotopy groups, which is to say

$$
\pi_{k}\left(G^{-\infty}(X ; E)\right) \cong \pi_{k}\left(\mathcal{G}^{-\infty}\right) \cong\left\{\begin{array}{cc}
\{0\} & k \text { even } \\
\mathbb{Z} & k \text { odd }
\end{array}\right.
$$

This periodicity in the homotopy groups is an instance of Bott periodicity as originally 
described by Bott in [4].

In this thesis, we will describe how Bott periodicity arises when one considers instead fibred cusp operators $\Psi_{\Phi}^{*}(X ; E)$ on a compact manifold with boundary $X$ acting on some complex vector bundle $E$. These operators were introduced by Mazzeo and Melrose in [12]. The definition involves a defining function for the boundary $\partial X$ and a fibration $\Phi: \partial X \rightarrow Y$ of the boundary. Again, one considers the group

$$
G_{\Phi}^{-\infty}(X ; E)=\left\{\operatorname{Id}+Q \mid Q \in \Psi_{\Phi}^{-\infty}(X ; E), \quad \operatorname{Id}+Q \text { is invertible }\right\}
$$

of invertible smoothing perturbations of the identity. As before, it has a $\mathcal{C}^{\infty}$-topology induced by the identification of smoothing operators with their Schwartz kernels. Our main result, stated in theorem $\mathbf{7 . 1 9}$, is to describe the homotopy groups of $G_{\Phi}^{-\infty}(X ; E)$ in terms of the $K$-theory of $T^{*} Y$, namely, all the even homotopy groups are shown to be isomorphic to the kernel of the topological index map

$$
\operatorname{ind}_{t}: K_{c}^{0}\left(T^{*} Y\right) \rightarrow \mathbb{Z}
$$

while all the odd homotopy groups are shown to be isomorphic to $\widetilde{K}^{-1}\left(Y^{T^{*} Y}\right)$, where $Y^{T^{*} Y}$ is the Thom space associated to the vector bundle $T^{*} Y$. This periodicity of the homotopy groups is what we interpret as Bott periodicity for fibred cusp operators. Strictly speaking, this result is only true when the fibres of the fibration $\Phi: \partial X \rightarrow Y$ are of dimension at least one, but in the particular case where $\Phi: \partial X \rightarrow Y$ is a finite covering, which includes the case of scattering operators, the result is still true provided one allows some stabilization (see the discussion at the beginning of chapter 7).

This is in a certain sense a generalization of proposition 3.6 in [18], where it was shown that, in the particular case where $\Phi: \partial X \rightarrow \mathrm{pt}$ is a trivial fibration (the case of cusp operators), all the homotopy groups of $G_{\Phi}^{-\infty}(X ; E)$ are trivial. This weak contractibility was used in [18] to derive a relative index theorem for families of elliptic cusp pseudodifferential operators. However, this relative index theorem does not seem to generalize in a simple way to fibred cusp operators with a non-trivial fibration $\Phi: \partial X \rightarrow Y$, but we still hope that the knowledge of the homotopy groups of $G_{\Phi}^{-\infty}(X ; E)$ will turn out to be useful in the understanding of the index of general Fredholm fibred cusp operators.

An important feature of fibred cusp operators is that smoothing operators are not necessarily compact. As a consequence, smoothing perturbations of the identity are not necessarily Fredholm, and when they are, they do not necessarily have a vanishing index. Our computation of the homotopy groups of $G_{\Phi}^{-\infty}(X ; E)$ relies on a careful study of the space

$$
\mathcal{F}_{\Phi}^{-\infty}(X ; E)=\left\{\operatorname{Id}+Q \mid Q \in \Psi_{\Phi}^{-\infty}(X ; E) \quad \text { Id }+Q \text { is Fredholm }\right\}
$$

of Fredholm smoothing perturbations of the identity. The second important result of this thesis, stated in theorem 6.6, is that the index of a Fredholm operator $(\operatorname{Id}+Q) \in \mathcal{F}_{\Phi}^{-\infty}(X ; E)$ can be described in terms of an associated $K$-class $\kappa(\operatorname{Id}+Q) \in$ 
$K_{c}^{0}\left(T^{*} Y\right)$, namely

$$
\operatorname{ind}(\operatorname{Id}+Q)=\operatorname{ind}_{t}(\kappa(\operatorname{Id}+Q)),
$$

where $\operatorname{ind}_{t}$ is the topological index introduced by Atiyah and Singer in [2]. When $\Phi: \partial X \rightarrow Y$ is not a finite covering, an important corollary is that the index map

$$
\text { ind }: \mathcal{F}_{\Phi}^{-\infty}(X ; E) \rightarrow \mathbb{Z}
$$

is surjective. However, let us emphasize that the topology of $\mathcal{F}_{\Phi}^{-\infty}(X ; E)$ is in general significantly different from the topology of the space $\mathcal{F}(X ; E)$ of all Fredholm operators acting on $\mathrm{L}^{2}(X ; E)$. Indeed, $\mathcal{F}(X ; E)$, which is a classifying space for even $K$-theory, has trivial odd homotopy groups and even homotopy groups isomorphic to $\mathbb{Z}$, while $\mathcal{F}_{\Phi}^{-\infty}(X ; E)$, as described in proposition 7.6 below, has even homotopy groups isomorphic to $K_{c}^{0}\left(T^{*} Y\right)$ and odd homotopy groups isomorphic to $\widetilde{K}^{-1}\left(Y^{T^{*} Y}\right)$. Nevertheless, in the particular case of cusp operators $(Y=\mathrm{pt})$, this shows that $\mathcal{F}_{\Phi}^{-\infty}(X ; E) \subset \mathcal{F}(X ; E)$ is quite big and is also a classifying space for even $K$-theory.

The thesis is organized as follows. We first briefly review $K$-theory and index theory in chapter 2. This gives the occasion to provide a detailed proof of the standard fact that, for classical pseudodifferential operators on a closed manifold, the group of invertible smoothing perturbations of the identity is a classifying space for odd $K$-theory. In chapter 3 , we review the definition and the main properties of fibred cusp operators. We then recall in chapter 4 the notion of a regularized trace on smoothing fibred cusp operators and its associated trace-defect formula. This is used to get an analytic formula for the index of operators in $\mathcal{F}_{\Phi}^{-\infty}(X ; E)$. In chapter 5 , we use spectral sections to associate a $K$-class to any Fredholm operator in $\mathcal{F}_{\Phi}^{-\infty}(X ; E)$. This $K$-class is in turn used in chapter 6 to get a topological index formula. In the case where $Y=\mathbb{S}^{1}$, we also provided an alternative proof using the analytic formula of section 4.2. This topological index will allow us, in chapter 7, to present the main result of this thesis, that is, the periodicity of the homotopy groups of $G_{\Phi}^{-\infty}(X ; E)$. Finally, we also discuss the relation of our result with [18] and show that, once the weak contractibility is known, for instance in the case of cusp operators, it is not much harder, adapting a proof of Kuiper in [8], to deduce the actual contractibility, this last improvement being more esthetic than useful. 


\section{Chapter 2}

\section{$K$-theory and Bott periodicity}

The purpose of this chapter is to review $K$-theory and explain its relation with Bott periodicity. We will also discuss how $K$-theory is used to study the index of an elliptic operator. Finally, we will recall how Bott periodicity arises in the context of classical pseudodifferential operators on a closed manifold. For further details about $K$-theory, we refer to the book of Atiyah [1].

\section{$2.1 \quad K$-theory}

Let $M$ be a topological space. Later on, $M$ will always be a smooth manifold, possibly with boundary or corners.

Definition 2.1. We denote by $\operatorname{Vect}(M)$ the set of isomorphism classes of complex vector bundles over $M$. It has the structure of a semigroup with addition given by the direct sum. It is also a semi-ring with multiplication given by the tensor product.

If $A$ is a semigroup, let $F(A)$ be the free abelian group generated by the elements of $A$ and let $E(A)$ be the subgroup of $F(A)$ generated by the elements of the form

$$
a+a^{\prime}-\left(a \oplus a^{\prime}\right), \quad a, a^{\prime} \in A,
$$

where $\oplus$ is the addition in $A$. To $A$, we can then associate the group

$$
K(A):=\frac{F(A)}{E(A)} .
$$

There is an obvious semigroup homomorphism $\alpha: A \rightarrow K(A)$ given by the composition of the inclusion $A \hookrightarrow F(A)$ with the quotient map $F(A) \rightarrow K(A)$. The group $K(A)$ has the universal property that if $G$ is any group, $\beta: A \rightarrow G$ any semigroup homomorphism, then there is a unique group homomorphism $\gamma: K(A) \rightarrow G$ such that $\beta=\gamma \circ \alpha$. Clearly, $K(A)$ is the unique group with this universal property. Notice also that if $A$ is a semi-ring, then $K(A)$ is obviously a ring.

Definition 2.2. If $M$ is a topological space, we write $K(M)$ for the ring $K(\operatorname{Vect}(M))$. 
This is the (even) $K$-theory of $M$. If $V \in \operatorname{Vect}(M)$, we will write $[V]$ for the image of $V$ in $K(M)$.

There is an alternative definition of $K(A)$ which goes as follows. Let $\Delta: A \rightarrow$ $A \times A$ be the diagonal homomorphism and define $K(A)$ to be the quotient $\frac{A \times A}{\Delta(A)}$. It is a group, since the inverse of $(a, b) \in K(A)$ is given by $(b, a)$. The semigroup homomorphism $\alpha_{A}: A \rightarrow K(A)$ is given by the composition of $a \mapsto(a, 0)$ with the quotient map $A \times A \rightarrow K(A)$. The pair $\left(K(A), \alpha_{A}\right)$ is a functor, so that if $\beta: A \rightarrow B$ is a semigroup homomorphism, there is a commutative diagram

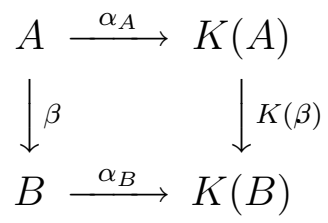

If $B$ is a group, then $\alpha_{B}$ is an isomorphism, which shows that $K(A)$ has the required universal property.

This alternative definition of $K(A)$ indicates that any element of $K(M)$ can be written in the form $[V]-[W]$, where $V$ and $W$ are two complex vector bundles on $M$. Let $\underline{\mathbb{C}^{n}}$ denote the trival bundle of rank $n$. Then for $n$ large enough, there exists $G \in \operatorname{Vect}(M)$ such that $F \oplus G \cong \mathbb{C}^{n}$ (see corollary 1.4.14 in [1]). Thus,

$$
[E]-[F]=[E]+[G]-([F]+[G])=[E]+[G]-\underline{\mathbb{C}^{n}} .
$$

This means that any element of $K(M)$ can be written in the form $[H]-\left[\underline{\mathbb{C}^{n}}\right]$ for some $H \in \operatorname{Vect}(M)$ and some $n \in \mathbb{N}_{0}$.

Definition 2.3. Two vector bundles $E$ and $F$ on a topological space $M$ are said to be stably equivalent if there exists $n \in \mathbb{N}_{0}$ such that

$$
E \oplus \underline{\mathbb{C}^{n}} \cong F \oplus \underline{\mathbb{C}^{n}}
$$

It turns out that two vector bundles $E$ and $F$ are stably equivalent if and only if $[E]=[F]$ in $K(M)$. Indeed, the "only if" part is clear. On the other hand, if $[E]=[F]$, then by the alternative definition of $K(M)$, there exists $G \in \operatorname{Vect}(M)$ such that

$$
E \oplus G \cong F \oplus G
$$

Hence, if $G^{\prime}$ is such that $G \oplus G^{\prime} \cong \underline{\mathbb{C}^{n}}$, we get

$$
E \oplus \underline{\mathbb{C}^{n}} \cong E \oplus G \oplus G^{\prime} \cong F \oplus G \oplus G^{\prime} \cong F \oplus \underline{\mathbb{C}^{n}},
$$

that is, $E$ and $F$ are stably equivalent.

When $f: M \rightarrow N$ is a continuous map, the pull-back map $f^{*}: \operatorname{Vect}(N) \rightarrow$ $\operatorname{Vect}(M)$ induces a ring homomorphism $f^{*}: K(N) \rightarrow K(M)$. This homomorphism depends only on the homotopy class of $f$. 
From now on, we will restrict our attention to compact topological spaces. Our objective will be to define $K$-theory for pairs of compact topological spaces. Let $\mathcal{C}$ denote the category of compact spaces, $\mathcal{C}^{+}$the category of compact spaces with distinguished basepoint, and $\mathcal{C}^{2}$ the category of compact pairs.

Definition 2.4. If $M \in \mathcal{C}^{+}$, we define the reduced $K$-theory $\widetilde{K}(M)$ to be the kernel of the homomorphism $i^{*}: K(M) \rightarrow K\left(m_{0}\right)$, where $i: m_{0} \hookrightarrow M$ is the inclusion of the distinguished basepoint. There is a splitting $K(M) \cong \widetilde{K}(M) \oplus K\left(m_{0}\right)$ induced by the collapsing map $c: M \rightarrow m_{0}$.

We can extend this definition to compact pairs as follows. We consider the functor

$$
\mathcal{C}^{2} \rightarrow \mathcal{C}^{+}
$$

which to a pair $(M, N) \in \mathcal{C}^{2}$ associates the space $M / N$ with basepoint $N / N$ (if $N=\emptyset$, $M / N$ is the disjoint union of $M$ with a point).

Definition 2.5. If $(M, N) \in \mathcal{C}^{2}$, we define the $K$-theory of the pair $(M, N)$ to be

$$
K(M, N):=\widetilde{K}(M / N)
$$

One can also consider the functor $\mathcal{C} \rightarrow \mathcal{C}^{+}$which to $M \in \mathcal{C}$ associates the space

$$
M^{+}=M / \emptyset
$$

which is the disjoint union of $\mathrm{M}$ with a point (the basepoint). Clearly, one has that $\widetilde{K}\left(M^{+}\right) \cong K(M)$. This will be useful to define odd $K$-theory. But we need first to discuss the smash product and the suspension.

Definition 2.6. Let $M, N \in \mathcal{C}^{+}$be compact spaces with distinguished basepoint. We define the smash product of $M$ and $N$, denoted $M \wedge N$, to be the space in $\mathcal{C}^{+}$given by

$$
M \wedge N:=\frac{M \times N}{\left\{m_{0}\right\} \times N \cup M \times\left\{n_{0}\right\}}
$$

where $m_{0}$ and $n_{0}$ are the basepoints of $M$ and $N$ respectively.

Given spaces $X, Y, Z \in \mathcal{C}^{+}$, one can check that there is a homeomorphism

$$
X \wedge(Y \wedge Z) \cong(X \wedge Y) \wedge Z
$$

In other words, the smash product is associative (modulo homeomorphisms). One can also check that it is commutative (modulo homeomorphisms).

An interesting example is to consider the iterated smash product of the circle with itself. Let $\mathbb{S}^{1}:=I / \partial I$ be our standard model for the circle, where $I=[0,1]$ is the unit interval. More generally, let $\mathbb{S}^{n}:=I^{n} / \partial I^{n}$ be our standard model for the 
sphere of dimension $n$, where $I^{n}$ is the unit cube in $\mathbb{R}^{n}$. Then there is a natural homeomorphism

$$
\mathbb{S}^{n} \cong \underbrace{\mathbb{S}^{1} \wedge \ldots \wedge \mathbb{S}^{1}}_{\mathrm{n} \text { times }} .
$$

Definition 2.7. Given $M \in \mathcal{C}^{+}$, we define the reduced suspension of $M$ to be the space

$$
S M:=\mathbb{S}^{1} \wedge M
$$

The $n$-th iterated suspension of $M$ is homeomorphic to $\mathbb{S}^{n} \wedge M$ and we will write it briefly as $S^{n} M$.

We can now introduce suspended versions of $K$-theory.

Definition 2.8. For $n \in \mathbb{N}_{0}$, we define the following rings:

$$
\begin{aligned}
\widetilde{K}^{-n}(M) & :=\widetilde{K}\left(S^{n} M\right), \quad \text { for } M \in \mathcal{C}^{+}, \\
K^{-n}(M, N) & :=\widetilde{K}^{-n}(M / N), \quad \text { for }(M, N) \in \mathcal{C}^{2}, \\
K^{-n}(M) & :=K^{-n}(M, \emptyset)=\widetilde{K}^{-n}\left(M^{+}\right), \quad \text { for } M \in \mathcal{C} .
\end{aligned}
$$

A fundamental result of $K$-theory is the periodicity theorem.

Theorem 2.9. (periodicity) For $n \in \mathbb{N}_{0}$,

$$
\begin{aligned}
\widetilde{K}^{-n-2}(M) & \cong \widetilde{K}^{-n}(M), \quad \text { for } M \in \mathcal{C}^{+}, \\
K^{-n-2}(M, N) & \cong K^{-n}(M, N), \quad \text { for }(M, N) \in \mathcal{C}^{2}, \\
K^{-n-2}(M) & \cong K^{-n}(M), \quad \text { for } M \in \mathcal{C} .
\end{aligned}
$$

For a proof of this theorem, we refer to [1]. It is intimately related with Bott periodicity. The relation will be clear once we will have discussed the clutching construction.

Definition 2.10. For $M \in \mathcal{C}$, we define the cone of $M$ to be the space

$$
C M:=\frac{I \times M}{\{0\} \times M}
$$

By identifying $M$ with the subspace $\{1\} \times M$ of $C M$, we can define the unreduced suspension of $M$ to be the space $C M / M \in \mathcal{C}^{+}$.

Notice that the unreduced suspension is a functor from $\mathcal{C}$ to $\mathcal{C}^{+}$, while the reduced suspension is a functor from $\mathcal{C}^{+}$to $\mathcal{C}^{+}$. If $M \in \mathcal{C}^{+}$and $m_{0} \in M$ is its basepoint, then we have an inclusion

$$
I \cong C m_{0} / m_{0} \subset C M / M
$$

and the collapsing of $I$ in $C M / M$ gives $S M$, the reduced suspension of $M$. Since $I$ is contractible, the map $p^{*}: \operatorname{Vect}(S M) \rightarrow \operatorname{Vect}(C M / M)$ arising from the collapsing map 
$p: C M / M \rightarrow S M$ is an isomorphism. Consequently, we also have an isomorphism at the level of $K$-theory,

$$
K(S M) \cong K(C M / M), \quad \widetilde{K}(S M) \cong K(C M, M) .
$$

Thus, as far as $K$-theory is concerned, this means that when $M \in \mathcal{C}^{+}$, we can use $S M$ for both the reduced and unreduced suspension without problems.

An alternative construction of the unreduced suspension of $M \in \mathcal{C}$ is to define it as the space

$$
C M / M \cong C^{+}(M) \cup C^{-}(M)
$$

where

$$
C^{+}(M)=\frac{\left[0, \frac{1}{2}\right] \times M}{\{0\} \times M}, \quad C^{-}(M)=\frac{\left[\frac{1}{2}, 1\right] \times M}{\{1\} \times M},
$$

with $C^{+}(M) \cap C^{-}(M) \cong M$. Given a complex vector bundle $E$ of rank $n$ over $C M / M$, its restrictions to $C^{+}(M)$ and $C^{-}(M)$ are trivial bundles since both spaces are contractible. Thus, there exist vector bundle isomorphisms

$$
\varphi^{+}: C^{+}(M) \times\left.\underline{\mathbb{C}^{n}} \rightarrow E\right|_{C^{+}(M)}, \quad \varphi^{-}: C^{-}(M) \times\left.\underline{\mathbb{C}^{n}} \rightarrow E\right|_{C^{-}(M)} .
$$

On the interesection $M \cong C^{+}(M) \cap C^{-}(M)$, this gives a bundle map

$$
\left(\varphi^{+}\right)^{-1} \circ \varphi^{-}: M \times \underline{\mathbb{C}^{n}} \rightarrow M \times \underline{\mathbb{C}^{n}}
$$

which defines a map

$$
\varphi: M \rightarrow \operatorname{GL}(n, \mathbb{C}) .
$$

Since $C^{+}(M)$ and $C^{-}(M)$ are contractible, the homotopy classes of $\varphi^{+}$and $\varphi^{-}$are well-defined, and so the homotopy class of $\varphi$ is also well-defined. This gives us a map

$$
\theta: \operatorname{Vect}_{n}(C M / M) \rightarrow[M ; \operatorname{GL}(n, \mathbb{C})]
$$

where $\operatorname{Vect}_{n}(C M / M)$ is the set of isomorphism classes of complex vector bundles of rank $n$ over $C M / M$ and $[M ; \operatorname{GL}(n, \mathbb{C})]$ is the set of homotopy classes of maps from $M$ to $\mathrm{GL}(n, \mathbb{C})$.

Conversely, given a map $\varphi: M \rightarrow \mathrm{GL}(n, \mathbb{C})$, one can get a complex vector bundle of rank $n$ over $C M / M$ by applying the clutching construction. Precisely, let $E^{+}=C^{+}(M) \times \underline{\mathbb{C}^{n}}$ be the trivial bundle over $C^{+}(M)$ and let $E^{-}(M)=C^{-} \times \underline{\mathbb{C}^{n}}$ be the trivial bundle over $C^{-}(M)$. Then the vector bundle over $C M / M$ given by the clutching construction is

$$
E^{+} \cup_{\varphi} E^{-}:=E^{+} \cup E^{-} / \sim
$$

where $\sim$ is the equivalence relation

$$
e^{+} \sim e^{-} \quad \text { if } \quad \varphi\left(e^{-}\right)=e^{+}
$$


where $\left.e^{+} \in E^{+}\right|_{m},\left.e^{-} \in E^{-}\right|_{m}$ and $m \in C^{+}(M) \cap C^{-}(M)$. The isomorphism class of $E^{+} \cup_{\varphi} E^{-}$only depends on the homotopy class of $\varphi$. Clearly, the clutching construction is just the inverse of the map (2.2), so we get

Lemma 2.11. The clutching construction gives a bijective map

$$
[M, \operatorname{GL}(n, \mathbb{C})] \rightarrow \operatorname{Vect}_{n}(C M / M)
$$

For any $n \in \mathbb{N}$, there is an inclusion

$$
\begin{aligned}
i_{n}: \operatorname{GL}(n, \mathbb{C}) & \hookrightarrow \mathrm{GL}(n+1, \mathbb{C}) \\
A & \mapsto A \oplus \mathrm{Id} .
\end{aligned}
$$

In other words, the entries of the matrix $i_{n}(A)$ are given by

$$
i_{n}(A)_{i j}= \begin{cases}A_{i j} & \text { if } i, j \leq n \\ 1 & \text { if } i=j=n+1 \\ 0 & \text { otherwise }\end{cases}
$$

Thus, one can consider the direct limit

$$
\mathrm{GL}(\infty, \mathbb{C}):=\lim _{k \rightarrow \infty} \mathrm{GL}(k, \mathbb{C}) .
$$

Proposition 2.12. For $M \in \mathcal{C}^{+}$and $\varphi: M \rightarrow \operatorname{GL}(n, \mathbb{C})$, let $E_{\varphi}$ denotes the complex vector bundle given by the clutching construction of lemma 2.11. Then the map $\varphi \mapsto$ $\left[E_{\varphi}\right]-\left[\mathbb{\mathbb { C }}^{n}\right]$ induces a group isomorphism

$$
[M ; \mathrm{GL}(\infty, \mathbb{C})]=\lim _{n \rightarrow \infty}[M ; \mathrm{GL}(n, \mathbb{C})] \cong \widetilde{K}(C M / M) \cong \widetilde{K}(S M),
$$

where the group structure on the left is induced from the group structure of $\mathrm{GL}(\infty, \mathbb{C})$.

Proof. That it is a bijective map is clear from lemma 2.11, (2.1) and the remark above definition 2.3. That it is a group homomorphism follows from the homotopy connecting the two maps $\mathrm{GL}(n, \mathbb{C}) \times \mathrm{GL}(n, \mathbb{C}) \rightarrow \mathrm{GL}(2 n, \mathbb{C})$ given by

$$
A \times B \mapsto\left(\begin{array}{cc}
A & 0 \\
0 & B
\end{array}\right)
$$

and

$$
A \times B \mapsto\left(\begin{array}{cc}
A B & 0 \\
0 & \mathrm{Id}
\end{array}\right) .
$$

This homotopy is explicitly given by

$$
h_{t}(A \times B)=\left(\begin{array}{cc}
A & 0 \\
0 & \mathrm{Id}
\end{array}\right)\left(\begin{array}{cc}
\cos t & \sin t \\
-\sin t & \cos t
\end{array}\right)\left(\begin{array}{cc}
\mathrm{Id} & 0 \\
0 & B
\end{array}\right)\left(\begin{array}{cc}
\cos t & -\sin t \\
\sin t & \cos t
\end{array}\right) .
$$


One can reformulate this proposition as follows.

Corollary 2.13. The space $\mathrm{GL}(\infty, \mathbb{C})$ is a classifying space for odd $K$-theory, that is, for $M \in \mathcal{C}^{+}$,

$$
\widetilde{K}^{-1}(M) \cong[M ; \mathrm{GL}(\infty, \mathbb{C})] .
$$

One can then easily deduce Bott periodicity from the periodicity of $K$-theory.

Corollary 2.14. (Bott periodicity) The even homotopy groups of $\mathrm{GL}(\infty, \mathbb{C})$ are trivial, while the odd homotopy groups are isomorphic to $\mathbb{Z}$.

Proof. Since $\mathrm{GL}(\infty, \mathbb{C})$ is a topological group we have that ${ }^{1}$

$$
\pi_{k}(\mathrm{GL}(\infty, \mathbb{C})) \cong\left[\mathbb{S}^{k} ; \mathrm{GL}(\infty, \mathbb{C})\right] \cong \widetilde{K}^{-1}\left(\mathbb{S}^{k}\right)
$$

Hence, by the periodicity theorem 2.9, if $k$ is odd,

$$
\pi_{k}(\mathrm{GL}(\infty, \mathbb{C})) \cong \widetilde{K}^{-k}\left(S^{1}\right) \cong \widetilde{K}^{-1}\left(S^{1}\right) \cong K(\mathrm{pt}) \cong \mathbb{Z},
$$

while if $k>0$ is even,

$$
\pi_{k}(\mathrm{GL}(\infty, \mathbb{C})) \cong \widetilde{K}^{-k}\left(S^{1}\right) \cong \widetilde{K}^{0}\left(\mathbb{S}^{1}\right) \cong 0
$$

Finally,

$$
\pi_{0}(\mathrm{GL}(\infty, \mathbb{C})) \cong \widetilde{K}^{-1}(\mathrm{pt}) \cong K(C \mathrm{pt}, \mathrm{pt}) \cong 0
$$

It is also possible to go the other way around, that is, to obtain the periodicity of $K$-theory from Bott periodicity using corollary 2.13 .

\section{$2.2 \quad K$-theory and index theory}

As Atiyah and Singer showed in their famous papers [2] and [3], $K$-theory is a very powerful tool to study the index of an elliptic operator. In chapter 6 , our discussion heavily rely on this $K$-theoretic approach. Therefore, we thought it was appropriate to briefly review this important application of $K$-theory.

Let $M$ be a compact connected manifold and let $E$ and $F$ be two complex vector bundles over $M$. Let $\Psi^{m}(M ; E, F)$ denote the space of pseudodifferential operators of order $m$ acting from smooth sections of $E$ to smooth sections of $F$. Then there is a short exact sequence

$$
\Psi^{m-1}(M ; E, F) \hookrightarrow \Psi^{m}(M ; E, F) \stackrel{\sigma_{m}}{\rightarrow} \mathcal{C}^{\infty}\left(S^{*} M ; \operatorname{hom}(E, F) \otimes \mathcal{R}^{m}\right),
$$

\footnotetext{
${ }^{1}$ see theorem 16.9 in [21]
} 
where $\sigma_{m}$ is the symbol map, $S^{*} M:=T M \backslash 0 / \mathbb{R}^{+}$is the sphere bundle and $\mathcal{R}^{m}$ is the trivial complex line bundle on $S^{*} M$ with sections given by functions over $T X \backslash 0$ which are positively homogeneous of degree $m$.

Definition 2.15. An operator $P \in \Psi^{m}(M ; E, F)$ is said to be elliptic if its symbol $\sigma_{m}(P)$ is invertible.

From the short exact sequence (2.4), one sees that invertible pseudodifferential operators are elliptic. The converse is not true in general, but very closed to be true.

Proposition 2.16. An elliptic operator $P \in \Psi^{m}(M ; E, F)$ is Fredholm seen as a bounded linear operator

$$
P: \mathrm{H}^{m}(M ; E) \rightarrow \mathrm{L}^{2}(M ; F)
$$

where

$$
\mathrm{H}^{m}(M ; E)=\left\{u \in \mathrm{L}^{2}(M ; F) \quad \mid \quad Q u \in \mathrm{L}^{2}(M ; E) \forall Q \in \Psi^{m}(M ; E)\right\}
$$

is the Sobolev space of order $m$ of sections of $E$. In other words, the range of $P$ is closed and both the kernel and the cokernel of $P$ are finite dimensional.

Definition 2.17. The index of an elliptic operator $P \in \Psi^{m}(M ; E, F)$ is its index seen as a Fredholm operator

$$
\operatorname{ind}(P)=\operatorname{dim}(\operatorname{ker} P)-\operatorname{dim}(\operatorname{coker} P) .
$$

As discussed in the following proposition, the index of an elliptic operators measures to some extent an obtruction to its invertibility.

Proposition 2.18. If $P \in \Psi^{m}(M ; E, F)$ is an elliptic operator, then there exists $Q \in \Psi^{-\infty}(M ; E, F)$ such that $P+Q$ is invertible if and only if $\operatorname{ind}(P)=0$.

So in particular, an elliptic operator with non-zero index is never invertible. Notice however that an elliptic operator with index zero is not necessarily invertible. What the proposition says is that it is always possible to perturb it (without changing the symbol) so that it becomes invertible.

The great achievement of Atiyah and Singer was to express the index of elliptic operators in terms of topological data.

Theorem 2.19. (Atiyah-Singer) The index of an elliptic pseudodifferential operator $P$ in $\Psi^{m}(M ; E, F)$ is given by the formula

$$
\operatorname{ind}(P)=(-1)^{\operatorname{dim}(M)} \int_{T M} \operatorname{ch}(\sigma(P)) \mathcal{T}(M)
$$

where $\mathcal{T}(M)$ is the Todd class of $T M$ and $\operatorname{ch}(\sigma(P))$ is the Chern character associated to the symbol of $P$. 
This theorem is extremely general in that it contains as particular cases the Riemann-Roch theorem, the Hirzebruch's signature theorem and the Gauss-Bonnet theorem. It has also many important applications, for instance in Gromov-Witten theory and in Seiberg-Witten theory, where it is used to compute the dimension of moduli spaces. Nowadays, there are various ways of proving this theorem, for example there is an analytic proof using the heat kernel. The advantage of the $K$-theoretic proof is that, although it requires more preparation, it provides a framework which makes the statement of the theorem natural and leads more directly to generalizations such as the equivariant index theorem and the family index theorem.

To show how $K$-theory comes into play, we first need to introduce compactly supported $K$-theory.

Definition 2.20. Let $M$ be a closed manifold and let $E \rightarrow M$ be a (real or complex) vector bundle over $M$. The Thom space of $E$, denoted $M^{E}$, is the one point compactification of $E$. The Thom space of $E$ can be seen as an element of $\mathcal{C}^{+}$by choosing its basepoint to be the point at infinity. We define the compactly supported $K$ theory of $E$ to be

$$
K_{c}^{0}(E):=\widetilde{K}^{0}\left(M^{E}\right) .
$$

This compactly supported $K$-theory is important for the following reason.

Proposition 2.21. The homotopy class of the symbol of an elliptic operator $P \in$ $\Psi^{m}(M ; E, F)$ gives rise to a well-defined element $k_{P} \in K_{c}^{0}\left(T^{*} M\right)$.

It turns out that this $K$-class $k_{P} \in K_{c}^{0}\left(T^{*} M\right)$ contains all the information about the index of the elliptic operator $P$. In fact, Atiyah and Singer defined in [2] an homomorphism of rings

$$
\operatorname{ind}_{t}: K_{c}^{0}\left(T^{*} M\right) \rightarrow \mathbb{Z}
$$

called the topological index map, which can be used to extract the index of $P$ out of the $K$-class $k_{P}$.

Theorem 2.22. (Atiyah-Singer)The index of an elliptic pseudodifferential operator $P \in \Psi^{m}(M ; E, F)$ is given by

$$
\operatorname{ind}(P)=\operatorname{ind}_{t}\left(k_{P}\right)
$$

where $k_{P} \in K_{c}^{0}\left(T^{*} M\right)$ is the $K$-class of proposition 2.21 .

From this theorem, it not too hard, using the Chern character map

$$
\operatorname{ch}: K_{c}^{0}\left(T^{*} M\right) \rightarrow \mathrm{H}_{c}^{*}\left(T^{*} M\right)
$$

and making a careful study of how it relates the Thom isomorphism of $K$-theory with the Thom isomorphism of cohomology, to get back the cohomological formula of theorem 2.19. To conclude this section, we will explain how to construct the 
topological index map (2.5). Since the cotangent bundle $T^{*} M$ is isomorphic to $T M$ via the use of some Riemannian metric, we have an isomorphism

$$
K_{c}^{0}\left(T^{*} M\right) \cong K_{c}^{0}(T M) .
$$

This isomorphism is independent of the choice of the Riemannian metric, since the homotopy class of the isomorphism $T^{*} M \cong T M$ is independent of the Riemannian metric chosen. Thus, whe might as well define the topological index map using the tangent bundle $T M$. Now, for $n \in \mathbb{N}$ large enough, there is an embedding $i: M \hookrightarrow \mathbb{R}^{n}$ of the closed manifold $M$. This in turn gives an embedding of the tangent bundle

$$
i_{*}: T M \hookrightarrow T \mathbb{R}^{n}=\mathbb{R}^{2 n} .
$$

If $N_{M}$ denotes the normal bundle of $M$ in $\mathbb{R}^{n}$, then the normal bundle of $T M$ in $\mathbb{R}^{2 n}$ is clearly isomorphic to $N \oplus N \cong N \otimes_{\mathbb{R}} \mathbb{C}$. Thus, one can see $N_{T M}$ as a complex vector bundle instead of a real vector bundle. There is therefore a well-defined Thom isomorphism

$$
\varphi: K_{c}^{0}(T M) \rightarrow K_{c}^{0}\left(N_{T M}\right)
$$

where $K_{c}^{0}\left(N_{T M}\right)$ is defined by thinking of $N_{T M}$ as a vector bundle over $M$. Identifying $N_{T M}$ with a tubular neighborhood of $M$ in $\mathbb{R}^{2 n}$, there is also a natural map

$$
k_{*}: K_{c}^{0}\left(N_{T M}\right) \rightarrow K_{c}^{0}\left(\mathbb{R}^{2 n}\right) .
$$

Intuitively, this map is a $K$-theoretic version of the natural inclusion

$$
\mathcal{C}_{c}^{\infty}\left(N_{T M}\right) \hookrightarrow \mathcal{C}_{c}^{\infty}\left(\mathbb{R}^{2 n}\right)
$$

obtained by extending functions to be zero outside $N_{T M}$. The composition of the maps (2.7) and (2.8) gives us a map

$$
i_{!}: K_{c}^{0}(T M) \rightarrow K_{c}^{0}\left(\mathbb{R}^{2 n}\right) .
$$

On the other hand, one can apply a similar construction to the inclusion $j:$ pt $\hookrightarrow \mathbb{R}^{n}$ of the origin in $\mathbb{R}^{n}$ to get a map

$$
j_{!}: K^{0}(\mathrm{pt}) \rightarrow K_{c}^{0}\left(\mathbb{R}^{2 n}\right)
$$

which is in fact an explicit isomorphism of the periodicity of $K$-theory. Since $K^{0}(\mathrm{pt})$ is canonically identifies with $\mathbb{Z}$, we can finally define the topological index map as follows.

Definition 2.23. The topological index $\boldsymbol{m a p} \operatorname{ind}_{t}: K_{c}^{0}(T M) \rightarrow \mathbb{Z}$ is defined by

$$
\operatorname{ind}_{t}=\left(j_{!}\right)^{-1} \circ i_{!}
$$

Of course, this construction involves many choices, but it turns out that the 
definition depends on none. We refer to [2] for details.

\subsection{Bott periodicity for classical pseudodifferential operators on a closed manifold}

Let $M$ be a closed manifold and $E \rightarrow M$ be a complex vector bundle over $M$. Consider the group

$$
G^{-\infty}(M ; E)=\left\{\operatorname{Id}+Q \mid Q \in \Psi^{-\infty}(M ; E), \quad \operatorname{Id}+Q \text { is invertible }\right\}
$$

of invertible smoothing perturbations of the identity. This becomes a topological group by taking the $\mathcal{C}^{\infty}$-topology induced by the identification of smoothing operators with their Schwartz kernels. Recall that the Schwartz kernel $K_{A}$ of $A \in \Psi^{-\infty}(M ; E)$ can be seen as an element of $\mathcal{C}^{\infty}(M \times M ; \operatorname{Hom}(E, E))$ where $\operatorname{Hom}(E, E)$ is the vector bundle over $M \times M$ with fiber at $\left(m_{1}, m_{2}\right) \in M \times M$ given by

$$
\left.\operatorname{Hom}(E, E)\right|_{\left(m_{1}, m_{2}\right)}=\operatorname{Hom}\left(E_{m_{2}}, E_{m_{1}}\right) \text {. }
$$

Then the action $A$ on $u \in \mathcal{C}^{\infty}(M ; E)$ is given by

$$
(A u)\left(m_{1}\right)=\int_{M} K_{A}\left(m_{1}, m_{2}\right) u\left(m_{2}\right) d g\left(m_{2}\right)
$$

where $d g$ is the volume form induced by some Riemannian metric $g$ on $M$.

Definition 2.24. Let $\mathcal{G}^{-\infty}$ be the topological group of invertible semi-infinite matrices $\delta_{i j}+\mathcal{Q}_{i j}$ such that

$$
\|\mathcal{Q}\|_{k}=\sum_{i, j \in \mathbb{N}}(i+j)^{k}\left|\mathcal{Q}_{i j}\right|<\infty, \quad \forall k \in \mathbb{N}_{0}
$$

the topology of $\mathcal{G}^{-\infty}$ being the one induced by the norms $\|\cdot\|_{k}, k \in \mathbb{N}_{0}$. Clearly, the group $\mathcal{G}^{-\infty}$ is a weak deformation retract ${ }^{2}$ of $\mathrm{GL}(\infty, \mathbb{C})$. In particular, it has the same homotopy groups.

The main result we would like to establish in this section is the following

Theorem 2.25. Their exists an isomorphism of topological groups

$$
G^{-\infty}(M ; E) \cong \mathcal{G}^{-\infty} .
$$

Thus, the homotopy groups of $G^{-\infty}(M ; E)$ are given by

$$
\pi_{k}\left(G^{-\infty}(M ; E)\right) \cong \pi_{k}\left(\mathcal{G}^{-\infty}\right) \cong\left\{\begin{array}{cl}
\{0\} & k \text { even } \\
\mathbb{Z} & k \text { odd }
\end{array}\right.
$$

\footnotetext{
${ }^{2}$ See definition 7.16.
} 
and $G^{-\infty}(M ; E)$ is a classifying space for odd $K$-theory

$$
\widetilde{K}^{-1}(N) \cong\left[N ; G^{-\infty}(M ; E)\right] \text { for } \quad N \in \mathcal{C}^{+} .
$$

We will break the proof in several lemmas. Let

$$
\Delta^{E}: \mathcal{C}^{\infty}(M ; E) \rightarrow \mathcal{C}^{\infty}(M ; E)
$$

be the Laplacian obtained by using the Riemannian metric $g$ on $M$ and choosing some metric and some connection on $E$. As a self-adjoint elliptic operator of order 2, the Laplacian $\Delta^{E}$ has a spectrum with many interesting properties.

Proposition 2.26. The spectrum of $\Delta^{E}$ is discrete and non-negative. Moreover, one can choose increasing eigenvalues $0=\lambda_{1}<\lambda_{2} \leq \lambda_{3} \leq \ldots$ with eigensections

$$
\Delta^{E} f_{k}=\lambda_{k} f_{k}, \quad f_{k} \in \mathcal{C}^{\infty}(M ; E),
$$

such that $\left\{f_{k}\right\}_{k \in \mathbb{N}}$ is an orthonormal basis of $\mathrm{L}^{2}(M ; E)$. Finally, there exists a constant $C_{\Delta^{E}}>0$ so that the asymptotic behavior of the eigenvalues of $\Delta^{E}$ is given by

$$
\lambda_{k} \sim C_{\Delta^{E}} k^{\frac{2}{n}}
$$

where $n=\operatorname{dim} M$. Here, $\lambda_{k} \sim C_{\Delta^{E}} k^{\frac{2}{n}}$ means that

$$
\lim _{k \rightarrow \infty} \frac{\lambda_{k}}{k^{\frac{2}{n}}}=C_{\Delta^{E}}
$$

For a proof of this proposition, we refer to theorem 15.2 in [20]. It allows us to construct the isomorphism of theorem 2.25 as follows. It is given by

$$
\begin{aligned}
f_{\Delta^{E}}: \quad G^{-\infty}(M ; E) & \rightarrow \mathcal{G}^{-\infty} \\
\mathrm{Id}+Q & \mapsto \delta_{i j}+\left\langle f_{i}, Q f_{j}\right\rangle .
\end{aligned}
$$

It is clearly a homomorphism of groups, but we still need to check that the image of $f_{\Delta^{E}}$ is really $\mathcal{G}^{-\infty}$ and that $f_{\Delta^{E}}$ is a homeomorphism. Furthermore, notice that it is by no way canonical, since it depends on the choice of the Laplacian $\Delta^{E}$.

Lemma 2.27. The image of $f_{\Delta^{E}}$ is contained in $\mathcal{G}^{-\infty}$.

Proof. It suffices to check that for all $Q \in \Psi^{-\infty}$, the semi-infinite matrix

$$
\mathcal{Q}_{i j}=\left\langle f_{i}, Q f_{j}\right\rangle_{\mathrm{L}^{2}}
$$

satisfies the estimates (2.11). The Schwartz kernel $K_{Q}$ of $Q$ is given by

$$
K_{Q}\left(m_{1}, m_{2}\right)=\sum_{i, j \in \mathbb{N}} \mathcal{Q}_{i j} f_{i}\left(m_{1}\right) \otimes g^{E}\left(\cdot, \overline{f\left(m_{2}\right)}\right) \in \mathcal{C}^{\infty}(M \times M ; \operatorname{Hom}(E, E)) .
$$


If $\mathcal{Q}$ were not satisfying the estimates of (2.11), then from the asymptotic behavior of the eigenvalues of $\Delta^{E}$, one would conclude that there exist $k, l \in \mathbb{N}$ such that

$$
P:=\left(\Delta^{E}\right)^{k} \circ Q \circ\left(\Delta^{E}\right)^{l}
$$

has Schwartz kernel

$$
\left.K_{P}\left(m_{1}, m_{2}\right)=\sum_{i, j} \mathcal{Q}_{i j} \lambda_{i}^{k} \lambda_{j}^{l} f_{i}\left(m_{1}\right) \otimes g^{E}\left(\cdot, \overline{f_{j}\left(m_{2}\right.}\right)\right)
$$

which is not in $\mathrm{L}^{2}(M \times M ; \operatorname{Hom}(E, E))$, so in particular is not smooth. But this would contradict the fact that $P=\left(\Delta^{E}\right)^{k} \circ Q \circ\left(\Delta^{E}\right)^{l}$ is a smoothing operator. This means $\mathcal{Q}$ must satisfies all the estimates in $(2.11)$ and hence $f_{\Delta^{E}}(\operatorname{Id}+Q) \in \mathcal{G}^{-\infty}$.

Now, let us recall some general result about elliptic operators.

Lemma 2.28. Let $P \in \Psi^{k}(M ; E)$ be an elliptic operator and $m \in \mathbb{Z}$. Then there exists a constant $C_{m}$ depending on $m$ such that for all $u \in H^{m+k}(M ; E)$, we have

$$
\|u\|_{\mathrm{H}^{m+k}} \leq C_{m}\left(\|P u\|_{\mathrm{H}^{m}}+\|u\|_{\mathrm{H}^{m}}\right)
$$

where $\|\cdot\|_{\mathrm{H}^{m}}$ denotes the norm in $\mathrm{H}^{m}(M ; E)$.

Proof. Let $G \in \Psi^{-k}(M ; E)$ be a parametrix for the operator $P$, so that

$$
G P=\operatorname{Id}+Q, \quad Q \in \Psi^{-\infty}(M ; E) .
$$

Then, given $u \in H^{m+k}(M ; E)$, we have that

$$
u=G P u-Q u \text {. }
$$

Since $\Psi^{-\infty}(M ; E) \subset \Psi^{-k}(M ; E)$, both $G$ and $Q$ are pseudodifferential operators of order $-k$, and hence they are continuous bounded linear operators from $\mathrm{H}^{m}(M ; E)$ to $\mathrm{H}^{m+k}(M ; E)$. Therefore, there exists a constant $C_{m}>0$ such that

$$
\begin{aligned}
\|u\|_{\mathrm{H}^{m+k}} & \leq\|G P u\|_{\mathrm{H}^{m+k}}+\|Q u\|_{\mathrm{H}^{m+k}} \\
& \leq C_{m}\left(\|P u\|_{\mathrm{H}^{m}}+\|u\|_{\mathrm{H}^{m}}\right)
\end{aligned}
$$

Lemma 2.29. The map $f_{\Delta^{E}}$ is bijective.

Proof. The map $f_{\Delta^{E}}$ is clearly injective, so we need to check that it is surjective. Let $\mathrm{Id}+\mathcal{Q} \in \mathcal{G}^{-\infty}$ be given. Then the operator $Q$ with Schwartz kernel

$$
K_{Q}\left(m_{1}, m_{2}\right)=\sum_{i, j} \mathcal{Q}_{i j} f_{i}\left(m_{1}\right) \otimes g^{E}\left(\cdot, \overline{f_{j}\left(m_{2}\right)}\right)
$$

will be such that $f_{\Delta^{E}}(\operatorname{Id}+Q)=\operatorname{Id}+\mathcal{Q}$. Thus, one has to check that $Q$ is a smoothing operator, or equivalently that $K_{Q}$ is smooth. Clearly, since the metric $g^{E}$ is smooth, 
the Schwartz kernel $K_{Q}$ will be smooth if and only if

$$
K_{Q}^{\prime}=\sum_{i, j} \mathcal{Q}_{i j} f_{i}\left(m_{1}\right) \otimes f_{j}\left(m_{2}\right)
$$

is smooth. So we need to show that $K_{Q}^{\prime}$ is smooth. As a first step, notice that since

$$
\sup _{i, j}\left|\mathcal{Q}_{i j}\right| \leq\|\mathcal{Q}\|_{0}
$$

one has that

$$
\left\|K_{Q}^{\prime}\right\|_{\mathrm{L}^{2}}^{2}=\sum_{i, j}\left|\mathcal{Q}_{i j}\right|^{2} \leq\|\mathcal{Q}\|_{0}\|\mathcal{Q}\|_{0} \leq\|\mathcal{Q}\|_{0}^{2}
$$

so $K_{Q}^{\prime} \in \mathrm{L}^{2}(M \times M ; E \times E)$. The remaining of the proof consists to apply lemma 2.28 recursively to improve to regularity of $K_{Q}^{\prime}$. But for this, we need a suitable elliptic operator. Let $\Delta_{1}^{E} \in \Psi^{2}(M \times M ; E \times E)$ be the operator obtained by making $\Delta^{E}$ acts on the first factor in the variable $m_{1}$, and similarly let $\Delta_{2}^{E} \in \Psi^{2}(M \times M ; E \times E)$ be the operator obtained by making $\Delta^{E}$ acts on the second factor in the variable $m_{2}$. Then

$$
P:=\Delta_{1}^{E}+\Delta_{2}^{E} \in \Psi^{2}(M \times M ; E \times E)
$$

is an elliptic operator with eigenvalues given by $\lambda_{i}+\lambda_{j}$ with $i, j \in \mathbb{N}$. Thus, we can apply lemma 2.28 to $u=K_{Q}^{\prime}$ with the elliptic operator $P$. We get

$$
\begin{aligned}
\left\|K_{Q}^{\prime}\right\|_{\mathrm{H}^{2}} & \leq C_{0}\left(\left\|P K_{Q}^{\prime}\right\|_{\mathrm{L}^{2}}+\left\|K_{Q}^{\prime}\right\|_{\mathrm{L}^{2}}\right) \\
& \leq C_{0}\left(\left(\sum_{i, j}\left|\mathcal{Q}_{i j}\right|^{2}\left(\lambda_{i}+\lambda_{j}\right)^{2}\right)^{\frac{1}{2}}+\left\|\mathcal{Q}_{0}\right\|\right)
\end{aligned}
$$

But since $\lambda_{k} \sim C_{\Delta^{E}} k^{\frac{2}{n}}$, we see that

$$
\left\|K_{Q}^{\prime}\right\|_{\mathrm{H}^{2}} \leq C_{0}^{\prime}\left(\left(\|\mathcal{Q}\|_{k_{0}}\|\mathcal{Q}\|_{0}\right)^{\frac{1}{2}}+\|\mathcal{Q}\|_{0}\right) \leq 2 C_{0}^{\prime}\|\mathcal{Q}\|_{k_{0}}
$$

for some constant $C_{0}^{\prime}>0$ and some $k_{0} \in \mathbb{N}$ large enough. More generally, applying recursively lemma 2.28 with $m \in 2 \mathbb{N}_{0}$, we get the estimates

$$
\left\|K_{Q}\right\|_{\mathrm{H}^{m+2}} \leq C_{m}^{\prime}\|\mathcal{Q}\|_{k_{m}}, \quad m \in 2 \mathbb{N}_{0}
$$

where $k_{m} \in \mathbb{N}$ and $C_{m}^{\prime}>0$ depend on $m$. By the Sobolev embedding theorem, this shows that $K_{Q}^{\prime}$ and $K_{Q}$ are smooth, and hence that $f_{\Delta^{E}}$ is bijective.

To conclude the proof of theorem 2.25, we need to show that $f_{\Delta^{E}}$ is an homeomorphism.

Lemma 2.30. The map $f_{\Delta^{E}}$ is a homeomorphism.

Proof. That $f_{\Delta^{E}}$ is continuous follows from the estimates (2.14) of the preceding proof and the Sobolev embedding theorem. To show that $f_{\Delta^{E}}^{-1}$ is continuous, first notice 
that the Hölder inequality gives us

$$
\begin{aligned}
\|\mathcal{Q}\|_{k} & =\sum_{i, j}\left|\mathcal{Q}_{i j}\right| \frac{(i+j)^{k+2}}{(i+j)^{2}} \\
& \leq\left(\sum_{i, j}\left|Q_{i j}\right|^{2}(i+j)^{2 k+4}\right)^{\frac{1}{2}}\left(\sum_{i, j} \frac{1}{(i+j)^{4}}\right)^{\frac{1}{2}} \\
& \leq C\left(\sum_{i, j}\left|Q_{i j}\right|^{2}(i+j)^{2 k+4}\right)^{\frac{1}{2}}
\end{aligned}
$$

where

$$
C=\left(\sum_{i, j} \frac{1}{(i+j)^{4}}\right)^{\frac{1}{2}}
$$

Hence, since $\lambda_{j} \sim C_{\Delta^{E}} j^{\frac{2}{n}}$, there exists a constant $C_{k}>0$ and $l_{k} \in \mathbb{N}$ so that

$$
\begin{aligned}
\|\mathcal{Q}\|_{k} & \leq C_{k}\left(\left(\sum_{i, j}\left|Q_{i j}\right|^{2}\left(\lambda_{i}+\lambda_{j}\right)^{2 l_{k}}\right)^{\frac{1}{2}}+\left(\sum_{i, j}\left|Q_{i j}\right|^{2}\right)^{\frac{1}{2}}\right) \\
& =C_{k}\left(\left\|P^{l_{k}} K_{Q}^{\prime}\right\|_{\mathrm{L}^{2}}+\left\|K_{Q}^{\prime}\right\|_{\mathrm{L}^{2}}\right) \\
& \leq C_{k}^{\prime}\left\|K_{Q}^{\prime}\right\|_{H^{2 l_{k}}} \leq C_{k}^{\prime \prime}\left\|K_{Q}\right\|_{\mathcal{C}^{2 l_{k}}}
\end{aligned}
$$

where $\|\cdot\|_{\mathcal{C}^{2 l_{k}}}$ is the $\mathcal{C}^{2 l_{k}}$-norm with respect to some Riemannian metric. From the estimate (2.15), we conclude that $f_{\Delta^{E}}^{-1}$ is continuous, so that $f_{\Delta^{E}}$ is a homeomorphism. 


\section{Chapter 3}

\section{Fibred Cusp Operators}

Since fibred cusp operators are the central object of study in this paper, let us recall briefly their definition and their main properties. A detailed description can be found in [12], where they were originally introduced. One can also look at section 2 of [11].

\subsection{The framework and the definition}

Let $X$ be a compact connected manifold with non-empty boundary $\partial X$. Assume that the boundary $\partial X$ has the structure of a (locally trivial) fibration

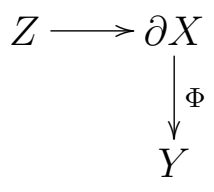

where $Y$ and $Z$ are closed manifolds, $Y$ being the base of the fibration and $Z$ being a typical fibre. We will not assume that the boundary $\partial X$ is connected, but if $Y$ is disconnected, we will assume that $Z$ is the same over all connected components of $Y$. For the disconnected case, we will basically use the generalization of [18] rather than the one presented in [12] (see remark 3.3 below). Let $x \in \mathcal{C}^{\infty}(X)$ be a defining function for the boundary $\partial X$, that is, $x$ is non-negative, $\partial X$ is the zero set of $x$ and $d x$ does not vanish anywhere on $\partial X$.

The defining function $x$ gives rise to an explicit trivialization of the conormal bundle of $\partial X$. A fibration (3.1) together with an explicit trivialization of the conormal bundle of $\partial X$ defines a fibred cusp structure. To a given fibred cusp structure, we will associate an algebra of cusp fibred operators denoted $\Psi_{\Phi}^{*}(X)$. Changing the trivialization of the conormal bundle of $\partial X$ will not change $\Psi_{\Phi}^{*}(X)$ drastically, since we will get an isomorphic algebra, but not in a canonical way. When we talk about an algebra of fibred cusp operators, we therefore tacitly assume that a defining function has been chosen. On the other hand, changing the fibration (3.1) has important consequences on the algebra $\Psi_{\Phi}^{*}(X)$. In particular, there are two extreme cases: the case where $Z=\{\mathrm{pt}\}$ and $\Phi=\mathrm{Id}$, which leads to the algebra of scattering operators, and the case where $Y=\{\mathrm{pt}\}$, which leads to the algebra of cusp operators. 
Definition 3.1. A fibred cusp vector field $V \in \mathcal{C}^{\infty}(X, T X)$ is a vector field which, at the boundary $\partial X$, is tangent to the fibres of the fibration $\Phi$, and such that $V x \in x^{2} \mathcal{C}^{\infty}(X)$, where $x$ is the defining function of the fibred cusp structure. We denote by $\mathcal{V}_{\Phi}(X)$ the Lie algebra of fibred cusp vector fields.

Let $(x, y, z)$ be coordinates in a neighborhood of $p \in \partial X \subset X$, where $x$ is the defining function for $\partial X$ and $y$ and $z$ are respectively local coordinates on $Y$ and $Z$ (we assume that the fibration $\Phi$ is trivial on the neighborhood of $p$ we consider). Then any fibred cusp vector field $V \in \mathcal{V}_{\Phi}(X)$ is locally of the form

$$
V=a x^{2} \frac{\partial}{\partial x}+\sum_{i=1}^{l} b_{i} x \frac{\partial}{\partial y^{i}}+\sum_{i=1}^{k} c_{i} \frac{\partial}{\partial z^{i}},
$$

where $l=\operatorname{dim} Y, k=\operatorname{dim} Z$ and $a, b_{i}, c_{i}$ are smooth functions.

Definition 3.2. The space of fibred cusp differential operators of order $m$, denoted $\operatorname{Diff}_{\Phi}^{m}(X)$, is the space of operators on $\mathcal{C}^{\infty}(X)$ generated by $\mathcal{C}^{\infty}(X)$ and products of up to $m$ elements of $\mathcal{V}_{\Phi}(X)$.

In the local coordinates $(x, y, z)$, a fibred cusp differential operator $P \in \operatorname{Diff}_{\Phi}^{m}(X)$ may be written as

$$
P=\sum_{|\alpha|+|\beta|+q \leq m} p_{\alpha, \beta, q}\left(x^{2} \frac{\partial}{\partial x}\right)^{q}\left(x \frac{\partial}{\partial y}\right)^{\alpha}\left(\frac{\partial}{\partial z}\right)^{\beta}
$$

where the $p_{\alpha, \beta, q}$ are smooth functions.

Intuitively, fibred cusp pseudodifferential operators are a generalization of definition 3.2 by allowing $P$ to be not only a polynomial in $x^{2} \frac{\partial}{\partial x}, x \frac{\partial}{\partial y}, \frac{\partial}{\partial z}$, but also a more general "function" in $x^{2} \frac{\partial}{\partial x}, x \frac{\partial}{\partial y}, \frac{\partial}{\partial z}$. The rigorous definition is however better understood by describing the Schwartz kernels of fibred cusp pseudodifferential operators.

Consider the cartesian product $X^{2}$ of the manifold $X$ with itself. This is a manifold with corner, the corner being $\partial X \times \partial X \subset X^{2}$. Schwartz kernels of fibred cusp pseudodifferential operators will be distributions on $X^{2}$. But all the subtleties of their behavior turn out to happen in the corner of $X^{2}$. To have a better picture of what is going on, one therefore blows up the corner in the sense of Melrose, [13], [14]. In fact, we need to blow up twice.

We first blow up the corner to get the space $X_{b}^{2}=\left[X^{2} ; \partial X \times \partial X\right]$. In [14], this space is used to describe the Schwartz kernels of $b$-pseudodifferential operators, where " $b$ " stands for boundary. For fibred cusp operators, we need to perform a second blow-up which depends on the fibration $\Phi$ we consider.

Beside the two old boundaries coming from $X^{2}, X_{b}^{2}$ has a new boundary called the front face. If $\beta_{b}: X_{b}^{2} \rightarrow X^{2}$ is the blow-down map, then this front face, denoted $\mathrm{ff}_{b}$, is given by

$$
\mathrm{ff}_{b}=\beta_{b}^{-1}(\partial X \times \partial X) \cong(\partial X \times \partial X) \times[-1,1]_{s}
$$

where $s=\frac{x-x^{\prime}}{x+x^{\prime}}$ and $x, x^{\prime}$ are the same boundary defining functions on the left and right factors of $X^{2}$. The function $s$ is well-defined on $X_{b}^{2}$. What we blow up next is 
the submanifold $B_{\Phi} \subset \mathrm{ff}_{b} \cong(\partial X \times \partial X) \times[-1,1]_{s}$ given by

$$
B_{\Phi}=\left\{\left(h, h^{\prime}, 0\right) \in \mathrm{ff}_{b} \mid \Phi(h)=\Phi\left(h^{\prime}\right)\right\} .
$$

Remark 3.3. When $\partial X$ is disconnected, we use the same definition for $B_{\Phi}$ and $\mathrm{ff}_{b}$. This is different from [12], where they consider smaller $B_{\Phi}$ and $\mathrm{ff}_{b}$ in the disconnected case. Using the same formal definition as in the connected case makes the generalization to the disconnected case straightforward.

So we consider the space $X_{\Phi}^{2}=\left[X_{b}^{2} ; B_{\Phi}\right]$. If $\beta_{\Phi}: X_{\Phi}^{2} \rightarrow X_{b}^{2}$ is the blow-down map, then the new boundary appearing on $X_{\Phi}^{2}$ is given by

$$
\mathrm{ff}_{\Phi}=\beta_{\Phi}^{-1}\left(B_{\Phi}\right)
$$

If $(y, z)$ and $\left(y^{\prime}, z^{\prime}\right)$ are local coordinates on the left and right factors of $(\partial X)^{2}$, then

$$
S=\frac{x-x^{\prime}}{x^{2}}, Y=\frac{y-y^{\prime}}{x}, z-z^{\prime}, x, y, z
$$

are local coordinates on $X_{\Phi}^{2}$, and in these coordinates, $\mathrm{ff}_{\Phi}$ occurs where $x=0$. Under the blow-down maps $\beta_{b}$ and $\beta_{\Phi}$, the diagonal $\Delta \subset \partial X \times \partial X$ lifts to $\Delta_{\Phi} \subset X_{\Phi}^{2}$. In the coordinates (3.7), it occurs where $S=Y=z-z^{\prime}=0$.

Definition 3.4. The fibred cusp density bundle ${ }^{\Phi} \Omega$ is the space of densities on $X$ which are locally of the form $\frac{\alpha}{r^{l+2}} d x d y d z$ near the boundary $\partial X$, where $\alpha$ is a smooth function and $l=\operatorname{dim} Y$. Let ${ }^{\Phi} \Omega_{R}$ be the lift of ${ }^{\Phi} \Omega$ to $X^{2}$ from the right factor. This gives the right fibred cusp density bundle ${ }^{\Phi} \Omega_{R}^{\prime}=\beta_{\Phi}^{*} \beta_{b}^{*}\left({ }^{\Phi} \Omega_{R}\right)$ on $X_{\Phi}^{2}$.

Definition 3.5. For any $m \in \mathbb{R}$, the space of $\Phi$-pseudodifferential operators of order $m$ is given by

$$
\Psi_{\Phi}^{m}(X)=\left\{k \in I^{m}\left(X_{\Phi}^{2}, \Delta_{\Phi} ;{ }^{\Phi} \Omega_{R}^{\prime}\right) \mid K \equiv 0 \text { at } \partial X_{\Phi}^{2} \backslash \mathrm{ff}_{\Phi}\right\}
$$

where $I^{m}\left(X_{\Phi}^{2}, \Delta_{\Phi} ;{ }^{\Phi} \Omega_{R}^{\prime}\right)$ denotes the space of conormal distributions (to $\Delta_{\Phi}$ ) of order $m$ (we refer to [12] and [7] for more details) and $\equiv$ denotes equality in Taylor series.

In local coordinates near the boundary, the action (near the diagonal) of cusp fibred pseudodifferential operators can be described as follows.

Proposition 3.6. If $\chi \in \mathcal{C}^{\infty}(X)$ has support in a coordinate patch $(\mathcal{U},(x, y, z))$ with $\mathcal{U} \cap \partial X \neq \emptyset$, where $x$ is the defining function of $\partial X$ and $y$ and $z$ are coordinates on $Y$ and $Z$, then the action of $P \in \Psi_{\Phi}^{m}(X)$ on $u \in \mathcal{C}_{c}^{\infty}(\mathcal{U})$ can be written

$$
(\chi P u)(x, y, z)=\int P_{\chi}\left(x, y, z, S, Y, z-z^{\prime}\right) \tilde{u}\left(x(1-x S), y-x Y, z^{\prime}\right) d S d Y d z^{\prime}
$$

where $S=\frac{x-x^{\prime}}{x^{2}}, Y=\frac{y-y^{\prime}}{x}, \tilde{u}$ is the coordinate representation of $u$ and $P_{\chi}$ is the restriction to $\mathcal{U} \times \mathbb{R}^{n}$ of a distribution on $\mathbb{R}^{n} \times \mathbb{R}^{n-k} \times \mathbb{R}^{k}$ which has compact support 
in the first and third variables, is conormal to $\{S=0, Y=0\} \times\left\{z=z^{\prime}\right\}$ and is rapidly decreasing with all derivatives as $|(S, Y)| \rightarrow \infty$.

\subsection{Some properties of fibred cusp operators}

If $E$ and $F$ are complex vector bundles on $X$, one can easily extend this definition to $\Phi$-pseudodifferential operators $\Psi_{\Phi}^{m}(X ; E, F)$ of order $m$ which act from $\mathcal{C}^{\infty}(X ; E)$ to $\mathcal{C}^{\infty}(X ; F)$.

Proposition 3.7. (Composition) For $m, n \in \mathbb{R}$ and $E, F, G$ complex vector bundles on $X$, we have that

$$
\Psi_{\Phi}^{m}(X ; F, G) \circ \Psi_{\Phi}^{n}(X ; E, F) \subset \Psi_{\Phi}^{m+n}(X ; E, G)
$$

As in the case of usual pseudodifferential operators, there is a notion of ellipticity.

Definition 3.8. The fibred cusp tangent bundle ${ }^{\Phi} T X$ is the smooth vector bundle on $X$ such that $\mathcal{V}_{\Phi}(X)=\mathcal{C}^{\infty}\left(X ;{ }^{\Phi} T X\right)$.

Notice that ${ }^{\Phi} T X$ is isomorphic to $T X$, although not in a natural way. Let ${ }^{\Phi} S^{*} X=$ $\left({ }^{\Phi} T X \backslash 0\right) / \mathbb{R}^{+}$denote the fibred cusp sphere bundle. Let $\mathcal{R}^{m}$ be the trivial complex line bundle on ${ }^{\Phi} S^{*} X$ with sections given by functions over ${ }^{\Phi} T X \backslash 0$ which are positively homogeneous of degree $m$.

Proposition 3.9. For all $m \in \mathbb{Z}$, there exists a symbol map

$$
\sigma_{m}: \Psi_{\Phi}^{m}(X ; E, F) \rightarrow \mathcal{C}^{\infty}\left({ }^{\Phi} S^{*} X ; \operatorname{hom}(E, F) \otimes \mathcal{R}^{m}\right)
$$

such that we have the following short exact sequence

$$
\Psi_{\Phi}^{m-1}(X ; E, F) \hookrightarrow \Psi_{\Phi}^{m}(X ; E, F) \stackrel{\sigma_{m}}{\rightarrow} \mathcal{C}^{\infty}\left({ }^{\Phi} S^{*} X ; \operatorname{hom}(E, F) \otimes \mathcal{R}^{m}\right) .
$$

Definition 3.10. If $P \in \Psi_{\Phi}^{m}(X ; E, F)$, we say that $\sigma_{m}(P)$ is the symbol of $P$. Moreover, if the symbol $\sigma_{m}(P)$ is invertible, we say that $P$ is elliptic.

As opposed to the case of usual pseudodifferential operators, ellipticity is not a sufficient condition to ensure that an operator is Fredholm. Some control near the boundary is also needed. Let ${ }^{\Phi} N Y \cong T Y \times \mathbb{R}$ be the null bundle on $Y$ of the restriction ${ }^{\Phi} T_{\partial X} X \rightarrow T X$.

Proposition 3.11. For any $m \in \mathbb{R}$, there is a short exact sequence

$$
x \Psi_{\Phi}^{m}(X ; E, F) \longrightarrow \Psi_{\Phi}^{m}(X ; E, F) \stackrel{N_{\Phi}}{\longrightarrow} \Psi_{\Phi s}^{m}(\partial X ; E, F)
$$


where $N_{\Phi}$ is the normal operator and $\Psi_{\Phi s}^{m}(\partial X ; E, F)$ is the space of ${ }^{\Phi} N Y$-suspended fibre pseudodifferential operators on $\partial X$ of order $m$ (see definition 2 in [12]). The normal operator is multiplicative, that is $N_{\Phi}(A) \circ N_{\Phi}(B)=N_{\Phi}(A \circ B)$. In terms of the Schwartz kernels, the normal operator $N_{\Phi}$ is just the restriction of the Schwartz kernel to the front face $\mathrm{ff}_{\Phi}$.

Definition 3.12. An operator $P \in \Psi_{\Phi}^{m}(X ; E, F)$ is said to be fully elliptic if it is elliptic and if $N_{\Phi}(P)$ is invertible.

Definition 3.13. Let $\mathrm{L}^{2}(X ; E)$ be the space of square integrable sections of $E$ with respect to some metric on $E$ and some smooth positive density on $X$. For $l \in \mathbb{R}$ and $m \geq 0$, we define the associated weighted Sobolev spaces by

$$
x^{l} \mathrm{H}_{\Phi}^{m}(X ; E)=\left\{u \in x^{l} \mathrm{~L}^{2}(X ; E) \mid P u \in x^{l} \mathrm{~L}^{2}(X ; E) \forall P \in \Psi_{\Phi}^{m}(X ; E)\right\},
$$

and

$$
\begin{aligned}
& x^{l} \mathrm{H}_{\Phi}^{-m}(X ; E)= \\
& \quad\left\{u \in \mathcal{C}^{-\infty}(X ; E) \mid u=\sum_{i=1}^{N} P_{i} u_{i}, u_{i} \in x^{l} \mathrm{~L}^{2}(X ; E), P_{i} \in \Psi_{\Phi}^{m}(X ; E)\right\} .
\end{aligned}
$$

Proposition 3.14. An operator $P \in \Psi_{\Phi}^{m}(X ; E, F)$ is Fredholm as a map

$$
P: x^{l} \mathrm{H}_{\Phi}^{m^{\prime}}(X ; E) \rightarrow x^{l} \mathrm{H}_{\Phi}^{m^{\prime}-m}(X ; F)
$$

for any $l, m \in \mathbb{R}$ if and only if it is fully elliptic.

Proposition 3.15. (elliptic regularity) The null space of a fully elliptic operator $P \in \Psi_{\Phi}^{m}(X ; E, F)$ is contained in $\dot{\mathcal{C}}^{\infty}(X ; E)$, the algebra of smooth sections of $E$ vanishing in Taylor series at the boundary $\partial X$.

It would be unfair to end this section without discussing in more details the space of suspended operators $\Psi_{\Phi s}^{*}(\partial X ; E, F)$. Since this paper is mostly concerned with smoothing operators, we will restrict our attention to $\Psi_{\Phi s}^{-\infty}(\partial X ; E, F)$. By taking the Fourier transform in the fibres of ${ }^{\Phi} N Y$, it is possible to see $P \in \Psi_{\Phi s}^{-\infty}(\partial X ; E, F)$ as a smooth family of smoothing operators parametrized by ${ }^{\Phi} N^{*} Y$, the dual of ${ }^{\Phi} N Y$. More precisely, consider the bundle

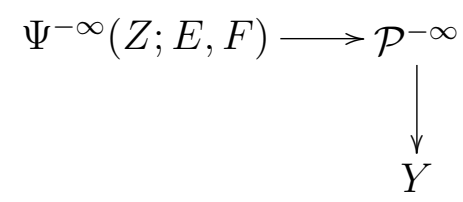

whose fibre at $y \in Y$ is given by $\Psi^{-\infty}\left(\Phi^{-1}(y) ;\left.E\right|_{\Phi^{-1}(y)},\left.F\right|_{\Phi^{-1}(y)}\right)$.

Remark 3.16. When $\operatorname{dim} Z=0$ and $\Phi: \partial X \rightarrow Y$ is a finite covering, the bundle $\mathcal{P}^{-\infty}$ is the vector bundle $\operatorname{hom}(\mathcal{E}, \mathcal{F})$, where $\mathcal{E} \rightarrow Y$ and $\mathcal{F} \rightarrow Y$ are the complex 
vector bundles over $Y$ with fibres at $y \in Y$ given by

$$
\mathcal{E}_{y}=\bigoplus_{z \in \Phi^{-1}(y)} E_{z}, \quad \mathcal{F}_{y}=\bigoplus_{z \in \Phi^{-1}(y)} F_{z}
$$

We can pull back $\mathcal{P}^{-\infty}$ on ${ }^{\Phi} N^{*} Y$ to get a bundle $\pi^{*} \mathcal{P}^{-\infty} \rightarrow{ }^{\Phi} N^{*} Y$, where

$$
\pi:{ }^{\Phi} N^{*} Y \rightarrow Y
$$

is the projection associated to the vector bundle ${ }^{\Phi} N^{*} Y$.

Proposition 3.17. There is a one-to-one correspondence between $\Psi_{\Phi s}^{-\infty}(\partial X ; E, F)$ and smooth sections of $\pi^{*} \mathcal{P}^{-\infty} \rightarrow{ }^{\Phi} N^{*} Y$ which are rapidly decreasing with all derivatives as one approaches infinity in ${ }^{\Phi} N^{*} Y \cong T^{*} Y \times \mathbb{R}$.

Remark 3.18. For $P \in \Psi_{\Phi s}^{m}(\partial X ; E, F)$, one has a similar correspondence, but one must replace $\pi^{*} \mathcal{P}^{-\infty}$ by the appropriate bundle $\pi^{*} \mathcal{P}^{m}$ of pseudodifferential operators of order $m$, while the sections at infinity should grow no faster than a homogeneous function of order $m$. The derivatives of the sections must also satisfy some growing conditions at infinity.

This is the point of view we will adopt for the rest of this thesis, that is, we will consider the Fourier transform of $N_{\Phi}(P)$, which is called the indicial family of $P$, instead of $N_{\Phi}(P)$. One advantage of doing so is that it is relatively easy to understand the indicial family in terms of local coordinates as in (3.7). If $P \in \Psi_{\Phi}^{-\infty}(X ; E, F)$ acts locally as in proposition 3.6 and if $\tau, \eta$ are the dual variables to $x, y$, then the indicial family is given by

$$
\widehat{N_{\Phi}(P)}(y, \eta, \tau)=\int e^{i \eta \cdot Y} e^{i \tau S} P\left(0, y, z, S, Y, z-z^{\prime}\right) d S d Y .
$$




\section{Chapter 4}

\section{An Analytical Formula for the Index}

We will now give an analytical formula for the index of Fredholm smoothing perturbations of the identity. To do this, we will need a trace-defect formula.

\subsection{The trace-defect formula}

For usual pseudodifferential operators on a closed manifold, it is a well-known fact that an operator will be of trace class provided its order is sufficiently low. For fibred cusp pseudodifferential operators, this is no longer true. Even smoothing operators are not necessarily of trace class. This is because there is a potential singularity at the boundary.

Let us concentrate on the case of smoothing operators. So we consider the space $\Psi_{\Phi}^{-\infty}(X ; E)$, where $E$ is some complex vector bundle on $X$. To avoid possible ambiguities, we assume that in a collar neighborhood $\partial X \times[0,1)_{x} \subset X$ of $\partial X$ parametrized by the defining function $x$, the vector bundle $E$ is of the form $E=\pi^{*} E_{\partial X}$, where $E_{\partial X}$ is a vector bundle on $\partial X$ and $\pi: \partial X \times[0,1)_{x} \rightarrow \partial X$ is the projection on the first factor. In other words, we choose an explicit trivialization of $E$ near the boundary which agrees with the choice of the defining function $x$. This way, we can make sense of Taylor series at the boundary in powers of $x$ (see (4.3) below).

If $A \in \Psi_{\Phi}^{-\infty}(X ; E)$ is of trace class, then its trace is given by integrating its Schwartz kernel along the diagonal

$$
\operatorname{Tr}(A)=\int_{\Delta_{\Phi}} \operatorname{tr}_{E} K_{A}=\int_{\Delta_{\Phi}} \operatorname{tr}_{E}\left(K_{A}^{\prime}\right) x^{-l-2} \nu, \quad K_{A}^{\prime} \in \mathcal{C}^{\infty}\left(X_{\Phi}^{2} ; \operatorname{Hom}(E, E)\right),
$$

where $l=\operatorname{dim} Y,\left(x^{\prime}\right)^{-l-2} \nu$ is a smooth section of the right fibred cusp density bundle ${ }^{\Phi} \Omega_{R}^{\prime}$, and $\operatorname{Hom}(E, E)$ is the pull back under the blow-down map $\beta_{\Phi} \circ \beta_{b}$ of the homomorphism bundle on $X^{2}$ with fibre at $\left(p, p^{\prime}\right) \in X^{2}$ given by $\operatorname{hom}\left(E_{p^{\prime}}, E_{p}\right)$. When restricted to the diagonal $\Delta \subset X^{2}, \operatorname{Hom}(E, E)$ is the same as $\operatorname{hom}(E, E)$.

The integral (4.1) does not converge for a general $A \in \Psi_{\Phi}^{-\infty}(X ; E)$, since $\nu$ is a smooth positive density when restricted to the diagonal $\Delta_{\Phi}$. To extend the trace to 
all smoothing operators, one has to subtract the source of divergence. To this end, consider the function

$$
z \mapsto \operatorname{Tr}\left(x^{z} A\right),
$$

which is a priori only defined for $\Re\{z\}>l+1$.

Lemma 4.1. The function $\operatorname{Tr}\left(x^{z} A\right)$ is holomorphic for $\Re\{z\}>l+1$ and it admits a meromorphic extension to the whole complex plane with at most simple poles at $l+1-\mathbb{N}_{0}$.

Proof. The holomorphy is clear. Recall from proposition 3.11 that in terms of the Schwartz kernels, the normal operator is the restriction to the front face $\mathrm{ff}_{\Phi}$. Let us consider instead the full normal operator $N_{\Phi}^{\prime}$ which gives us the full Taylor series of the Schwartz kernel at the front face $\mathrm{ff}_{\Phi}$, using $x$ as a defining function,

$$
N_{\Phi}^{\prime}(A)=\sum_{k=0}^{\infty} x^{k} A_{k}, \quad A_{k} \in \Psi_{\Phi s}^{-\infty}(\partial X ; E) .
$$

This Taylor series does not necessarily converge, so $N_{\Phi}^{\prime}(A)$ is really a formal power series in $x$ which contains all the information about the derivatives of $A$ at the front face $\mathrm{ff}_{\Phi}$. The Taylor series $N_{\Phi}^{\prime}(A)$ contains all the terms causing a divergence in the definition of $\operatorname{Tr}\left(x^{z} A\right)$. A simple computation shows that the function $\operatorname{Tr}\left(x^{z} x^{k} A_{k} \chi(x)\right)$, which is a priori only defined for $\Re\{z\}>l+1-k$, has a meromorphic extension to the whole complex plane with a single simple pole at $z=l+1-k$. Here, $\chi \in \mathcal{C}_{c}^{\infty}([0,+\infty))$ is some cut-off function with $\chi \equiv 1$ in a neighborhood of $x=0$. Thus, we see that $\operatorname{Tr}\left(x^{z} A\right)$ admits a meromorhic extension with at most simple poles at $l+1-\mathbb{N}_{0}$.

In particular, there is in general a pole at $z=0$ coming from the term $A_{l+1}$ in $(4.3)$.

Definition 4.2. For $A \in \Psi_{\Phi}^{-\infty}(X ; E)$, the residue trace $\operatorname{Tr}_{\mathrm{R}}(A)$ of $A$ is the residue at $z=0$ of the meromorphic function $z \mapsto \operatorname{Tr}\left(x^{z} A\right)$.

Using the local representation of proposition 3.6, the residue trace can be expressed as

$$
\operatorname{Tr}_{\mathrm{R}}(A)=\int \operatorname{tr}_{E}\left(A_{l+1}\left(y, z, S=0, Y=0, z-z^{\prime}=0\right)\right) d y d z .
$$

Taking the Fourier transform in the fibres of $N_{\Phi} Y \cong T Y \times \mathbb{R}$ as in (3.11), the residue trace can be rewritten as (cf. equations 4.1 and 4.2 in [15])

$$
\operatorname{Tr}_{\mathrm{R}}(A)=\frac{1}{(2 \pi)^{l+1}} \int_{N_{\Phi}^{*} Y} \operatorname{Tr}_{\Phi^{-1}(y)}\left(\widehat{A}_{l+1}(y, \tau, \eta)\right) d y d \eta d \tau
$$

where $\operatorname{Tr}_{\Phi^{-1}(y)}$ is the trace on $\Psi^{-\infty}\left(\Phi^{-1}(y) ;\left.E\right|_{\Phi^{-1}(y)}\right)$. Notice that this integral does not depend on the choice of coordinates on $Y$, since $d y d \eta$ is the volume form coming from the canonical symplectic form on $T^{*} Y$. Of course, $d \tau$ depends on the choice of the defining function $x$, but we assume it has been fixed. 
One can then extend the trace to all smoothing operators in $\Psi_{\Phi}^{-\infty}(X ; E)$ by subtracting the pole at $z=0$.

Definition 4.3. The regularized trace $\overline{\operatorname{Tr}}(A)$ of $A \in \Psi_{\Phi}^{-\infty}(X ; E)$ is given by

$$
\overline{\operatorname{Tr}}(A)=\lim _{z \rightarrow 0}\left(\operatorname{Tr}\left(x^{z} A\right)-\frac{\operatorname{Tr}_{\mathrm{R}}(A)}{z}\right) .
$$

Remark 4.4. For $A \in x^{l+2} \Psi_{\Phi}^{-\infty}(X ; E), A$ is of trace class and $\overline{\operatorname{Tr}}(A)=\operatorname{Tr}(A)$.

The regularized trace is defined for all $A \in \Psi_{\Phi}^{-\infty}(X ; E)$ and it is an extension of the usual trace, but it is not properly speaking a trace, since it does not vanish on commutators. The trace-defect formula measures how far the regularized trace is from being a trace.

Proposition 4.5. (Trace-defect formula) For $A, B \in \Psi_{\Phi}^{-\infty}(X ; E)$, the tracedefect is given by

$$
\overline{\operatorname{Tr}}([A, B])=\operatorname{Tr}_{\mathrm{R}}\left(\left(D_{\log x} A\right) B\right)
$$

where $D_{\log x} A=\left.\frac{\partial}{\partial z} A_{z}\right|_{z=0}$ and $A_{z}=x^{-z}\left[x^{z}, A\right]$.

Proof. For $\Re\{z\} \gg 0$, we have

$$
\begin{aligned}
\operatorname{Tr}\left(x^{z}[A, B]\right) & =\operatorname{Tr}\left(x^{z} A B-x^{z} B A\right)=\operatorname{Tr}\left(x^{z} A B\right)-\operatorname{Tr}\left(\left(x^{z} B\right) A\right) \\
& =\operatorname{Tr}\left(x^{z} A B\right)-\operatorname{Tr}\left(A x^{z} B\right)-\operatorname{Tr}\left(\left[x^{z} B, A\right]\right) .
\end{aligned}
$$

But $\operatorname{Tr}\left(\left[x^{z} B, A\right]\right)=0$ for $\Re\{z\} \gg 0$, so

$$
\begin{aligned}
\operatorname{Tr}\left(x^{z}[A, B]\right) & =\operatorname{Tr}\left(x^{z} A B\right)-\operatorname{Tr}\left(A x^{z} B\right) \\
& =\operatorname{Tr}\left(\left[x^{z}, A\right] B\right)=\operatorname{Tr}\left(x^{z} A_{z} B\right) .
\end{aligned}
$$

Here, $A_{z}=x^{-z}\left[x^{z}, A\right]$ is entire in $z$ and vanishes at $z=0$, so

$$
\operatorname{Tr}\left(x^{z}[A, B]\right)=z \operatorname{Tr}\left(x^{z}\left(D_{\log x} A\right) B\right)+\mathcal{O}(z), \quad \text { where } \quad D_{\log x} A=\left.\frac{\partial}{\partial z} A_{z}\right|_{z=0}
$$

Thus, we see that $\operatorname{Tr}\left(x^{z}[A, B]\right)$ has no pole at $z=0$ and its value at $z=0$ is given by

$$
\overline{\operatorname{Tr}}([A, B])=\operatorname{Tr}_{\mathrm{R}}\left(\left(D_{\log x} A\right) B\right) .
$$

In the case of cusp operators $(Y=\{\mathrm{pt}\})$, it is possible to use (4.5) to get an explicit formula depending only on the indicial families of $A$ and $B$ (cf. [18]). In the more general case, higher order terms of the Taylor series (4.3) are involved. Combined with the geometry of $Y$, this makes an explicit computation harder or maybe even impossible to get. 


\subsection{The index in terms of the trace-defect formula}

Let $\mathcal{F}_{\Phi}^{-\infty}(X ; E)$ denote the space of Fredholm operators of the form $\mathrm{Id}+A$ with $A \in \Psi_{\Phi}^{-\infty}(X ; E)$. From proposition 3.14 , the space $\mathcal{F}_{\Phi}^{-\infty}(X ; E)$ is given by

$$
\mathcal{F}_{\Phi}^{-\infty}(X ; E)=\left\{\operatorname{Id}+A \mid A \in \Psi_{\Phi}^{-\infty}(X ; E), \quad N_{\Phi}(\operatorname{Id}+A) \text { is invertible }\right\} .
$$

The goal of the next two chapters will be to obtain a topological description of the index of operators in $\mathcal{F}_{\Phi}^{-\infty}(X ; E)$, that is, a description in terms of $K$-theory. As a first step, let us use the trace-defect formula to get some insight about the index.

Notation 4.6. From now on we will follow the notation of (4.3) for the indicial family. So $\widehat{A}_{0}=\widehat{N_{\Phi}(A)}$ will denote the indicial family. In proposition 3.17, it is $\widehat{A}_{0}$ which can be seen as a section of the bundle $\pi^{*} \mathcal{P}^{-\infty}$.

Given $(\operatorname{Id}+A) \in \mathcal{F}_{\Phi}^{-\infty}(X ; E)$, let $(\operatorname{Id}+B) \in \mathcal{F}_{\Phi}^{-\infty}(X ; E)$ be a parametrix, that is

$$
(\operatorname{Id}+A)(\operatorname{Id}+B)=\operatorname{Id}+Q_{1}, \quad(\operatorname{Id}+B)(\operatorname{Id}+A)=\operatorname{Id}+Q_{2},
$$

with $Q_{1}, Q_{2} \in x^{\infty} \Psi_{\Phi}^{-\infty}(X ; E)$, where

$$
x^{\infty} \Psi_{\Phi}^{-\infty}(X ; E)=\left\{P \in \Psi_{\Phi}^{-\infty}(X ; E) \mid N_{\Phi}^{\prime}(P)=\sum_{k=0}^{\infty} x^{k} P_{k}=0\right\} .
$$

In particular, $Q_{1}$ and $Q_{2}$ are compact operators of trace class, so $[\operatorname{Id}+A, \operatorname{Id}+B]=$ $Q_{1}-Q_{2}$ is also of trace class. Using Calderón's formula for the index, we have that

$$
\begin{aligned}
\operatorname{ind}(\operatorname{Id}+A) & =\operatorname{Tr}([\operatorname{Id}+A, \operatorname{Id}+B]) \\
& =\overline{T r}([\operatorname{Id}+A, \operatorname{Id}+B]), \quad \text { by remark } 4.4 \\
& =\overline{T r}([A, B]) \\
& =\operatorname{Tr}_{\mathrm{R}}\left(\left(D_{\log x} A\right) B\right), \quad \text { by proposition } 4.5 .
\end{aligned}
$$

Using (4.5), we get the following formula for the index.

Proposition 4.7. The index of $(\operatorname{Id}+A) \in \mathcal{F}_{\Phi}^{-\infty}(X ; E)$ is given by

$$
\operatorname{ind}(\operatorname{Id}+A)=\frac{1}{(2 \pi)^{l+1}} \int_{\Phi_{N^{*} Y}} \operatorname{Tr}_{\Phi^{-1}(y)}\left(\left(\widehat{D_{\log x} A} B\right)_{l+1}(y, \tau, \eta)\right) d y d \eta d \tau
$$

where $(\operatorname{Id}+B) \in \mathcal{F}_{\Phi}^{-\infty}(X ; E)$ is a parametrix for $A$.

In the case of cusp operators, one can rewrite this formula in terms of the indicial family of $A$ only (see [18]). In fact, one can show without using the trace-defect formula that the index only depends on the indicial family.

Proposition 4.8. The index of $(\operatorname{Id}+A) \in \mathcal{F}_{\Phi}^{-\infty}(X ; E)$ only depends on the homotopy class of the indicial family $\mathrm{Id}+\widehat{A}_{0}$ seen as an invertible section of the bundle 
$\pi^{*} \mathcal{P}^{-\infty}$ of proposition 3.17 which converges rapidly to Id with all derivatives as one approaches infinity.

Proof. Suppose that $t \mapsto \mathrm{Id}+\widehat{A}_{0}(t), t \in[0,1]$ is a (smooth) homotopy of invertible indicial families with $\widehat{A}_{0}(t) \in \Psi_{\Phi s}^{-\infty}(\partial X ; E)$ for all $t \in[0,1]$. Suppose also that $\operatorname{Id}+\widehat{A}_{0}(0)$ and $\operatorname{Id}+\widehat{A}_{0}(1)$ are the indicial families of $(\operatorname{Id}+A(0)),(\operatorname{Id}+A(1)) \in$ $\mathcal{F}_{\Phi}^{-\infty}(X ; E)$. Thinking in terms of Schwartz kernels and using Seeley extension for manifolds with corners (see the proof of propostion 1.4.1 in [13]), we can construct a homotopy $t \mapsto A(t), t \in[0,1]$, joining $A(0)$ and $A(1)$, such that $A(t) \in \Psi_{\Phi}^{-\infty}(X ; E)$ and

$$
\left.\widehat{N_{\Phi}(A(t)}\right)=\widehat{A}_{0}(t) \forall t \in[0,1] .
$$

Since $t \mapsto \operatorname{Id}+\widehat{A}_{0}(t)$ is a homotopy of invertible indicial families, we know by proposition 3.14 that $t \mapsto(\mathrm{Id}+A(t))$ is a homotopy of Fredholm operators. By the homotopy invariance of the index, we conclude that

$$
\operatorname{ind}(\operatorname{Id}+A(0))=\operatorname{ind}(\operatorname{Id}+A(1)),
$$

which shows that the index of an operator $(\operatorname{Id}+A) \in \mathcal{F}_{\Phi}^{-\infty}(X ; E)$ only depends on the homotopy class of its indicial family.

One therefore expects that it is possible, at least in theory, to rewrite the formula of proposition 4.7 so that it only involves the indicial family. A first step in this direction is to notice that, by proposition 4.8, we can assume that the operator $\mathrm{Id}+A \in \mathcal{F}_{\Phi}^{-\infty}(X ; E)$ is such that

$$
\widehat{N_{\Phi}^{\prime}(A)}=\widehat{A_{0}} \text {, with } A_{k}=0 \forall k \in \mathbb{N} \text {. }
$$

However, once this assumption is made on $A$, it is not possible to assume the same for the parametrix $B$. To be more precise, the equation (4.10) completely determined $\widehat{N_{\Phi}^{\prime}(B)}$ in terms of $\widehat{N_{\Phi}^{\prime}(A)}$. This will become clear through the concept of $*$-product.

Definition 4.9. Let $\Psi_{\Phi s}^{-\infty}(\partial X ; E)[[x]]$ denote the space of formal power series in $x$ with coefficients taking values in $\Psi_{\Phi s}^{-\infty}(\partial X ; E)$.

Lemma 4.10. The full normal operator $N_{\Phi}^{\prime}: \Psi_{\Phi}^{-\infty}(X ; E) \rightarrow \Psi_{\Phi s}^{-\infty}(\partial X ; E)[[x]]$ is surjective.

Proof. This is an easy consequence of Seeley extension for manifolds with corners.

Definition 4.11. For $a, b \in \Psi_{\Phi s}^{-\infty}(\partial X ; E)[[x]]$, the $*$-product $a * b \in \Psi_{\Phi s}^{-\infty}(\partial X ; E)[[x]]$ is defined to be

$$
a * b=\widehat{N_{\Phi}^{\prime}(\widehat{A \circ} B)}
$$

where $A, B \in \Psi_{\Phi}^{-\infty}(X ; E)$ are chosen such that $\widehat{N_{\Phi}^{\prime}(A)}=a, \widehat{N_{\Phi}^{\prime}(B)}=b$. It does not depend on the choice of $A$ and $B$, since $x^{\infty} \Psi_{\Phi}^{-\infty}(X ; E)$ is an ideal of $\Psi_{\Phi}^{-\infty}(X ; E)$. 
Proposition 4.12. There exist differential operators $P_{k, j, l}$ and $Q_{k, j, l}$ acting on sections of the bundle $\mathcal{P}^{-\infty}$ defined in (3.10) so that, for any $a, b \in \Psi_{\Phi s}^{-\infty}(\partial X ; E)[[x]]$, the $*$-product of $a$ and $b$ is given by

$$
a * b=\left(\sum_{j=0}^{\infty} x^{j} a_{j}\right) *\left(\sum_{j=0}^{\infty} x^{j} b_{j}\right)=\sum_{k=1}^{\infty} \sum_{j+l \leq k} x^{k}\left(P_{k, j, l} a_{j}\right) \circ\left(Q_{k, j, l} b_{l}\right) .
$$

Moreover, if $\Phi: \partial X \rightarrow Y$ is a trivial fibration, then, once an explicit trivialization has been chosen, the differential operators $P_{k, j, l}$ and $Q_{k, j, l}$ are independent of the geometry of the typical fibre $Z$.

For the proof of proposition 4.12, we refer to proposition 3.11 in [10], where a similar result is obtained. In terms of the $*$-product, the equation (4.10) translates into

$$
\widehat{N_{\Phi}^{\prime}(A)}+\widehat{N_{\Phi}^{\prime}(B)}=-\widehat{N_{\Phi}^{\prime}(A)} * \widehat{N_{\Phi}^{\prime}(B)}=-\widehat{N_{\Phi}^{\prime}(B)} * \widehat{N_{\Phi}^{\prime}(A)} .
$$

Looking at this equation order by order in $x$ completely determines $\widehat{N_{\Phi}^{\prime}(B)}$ in terms of $\widehat{N_{\Phi}^{\prime}(A)}$. In general, for $k \in \mathbb{N}$, on can expect that $\widehat{B}_{k} \neq 0$, so $\widehat{N_{\Phi}^{\prime}(B)}$ will involve a term of order $x^{k}$. This is also the case for $D_{\log x} A$.

Lemma 4.13. If $\widehat{N_{\Phi}^{\prime}(A)}=\widehat{A}_{0}$, then

$$
\left.N_{\Phi}^{\prime} \widehat{\left(D_{\log x}\right.} A\right)=\sum_{k=1}^{\infty} \frac{x^{k} D_{\tau}^{k} \widehat{A}_{0}}{k}
$$

Proof. The Schwartz kernel of $A_{z}=x^{-z}\left[x^{z}, A\right]$ is $A\left(1-\left(\frac{x^{\prime}}{x}\right)^{z}\right)$, so the the Schwartz kernel of $D_{\log x} A$ is given by

$$
\left.\frac{\partial}{\partial z}\left(A\left(1-\left(\frac{x^{\prime}}{x}\right)^{z}\right)\right)\right|_{z=0}=-A \log \left(\frac{x^{\prime}}{x}\right) .
$$

But $\frac{x^{\prime}}{x}=1-S x$, where $S=\frac{x-x^{\prime}}{x^{2}}$, so

$$
D_{\log x} A=-A \log (1-S x)=A \sum_{k=1}^{\infty} \frac{(S x)^{k}}{k} .
$$

From $(3.11), \widehat{N_{\Phi}\left(S^{k} A\right)}=D_{\tau}^{k} \widehat{A_{0}}$, where $D_{\tau}=-i \frac{\partial}{\partial \tau}$, so we conclude that

$$
\left.N_{\Phi}^{\prime} \widehat{\left(D_{\log x}\right.} A\right)=\sum_{k=1}^{\infty} \frac{x^{k} D_{\tau}^{k} \widehat{A}_{0}}{k}
$$

Thus, knowing the differential operators $P_{k, j, l}$ and $Q_{k, j, l}$, we see that it is possible to rewrite the formula of proposition 4.7 into an expression involving only the indicial 
family of $A$. The problem of course is to actually find out what are those differential operators. This is relatively easy in the cusp case, but the case of an arbirary manifold $Y$ seems hopeless. Still, in the case where $Y=\mathbb{S}^{1}$, it is possible to perform some computation as we will see in section 6.3. From proposition 4.7 and proposition 4.12 , one can extract some important information about the index. For instance, notice that the formula does not involve the geometry of the interior of $X$, so if $X^{\prime}$ is another manifold with the same boundary as $X$ and if $\left(\mathrm{Id}+A^{\prime}\right) \in \mathcal{F}_{\Phi}^{-\infty}\left(X^{\prime} ; E\right)$ has the same indicial family as the one for $\operatorname{Id}+A$, then $\operatorname{Id}+A$ and $\operatorname{Id}+A^{\prime}$ have the same index. 


\section{Chapter 5}

\section{The Associated $K$-class}

We will now try to have a better understanding of the topological information contained in the indicial family. When the fibration $\Phi$ is trivial, it is almost straightforward to define a $K$-class out of the homotopy class of the indicial family. But to include the more general case of a non-trivial fibration, we first need to discuss the concept of spectral section.

\subsection{Spectral Sections}

Spectral sections were originally introduced in [17] to describe the boundary conditions for families of Dirac operators on manifolds with boundary. We intend to use spectral sections for a very different purpose, and instead of dealing with families of Dirac operators, we will consider families of Laplacians.

Let $\phi: M \rightarrow B$ be a smooth (locally trivial) fibration of compact manifolds, where the fibres are closed manifolds and the base $B$ is a compact manifold with possibly a non-empty boundary $\partial B$. Let $T(M / B) \subset T M$ denote the null space of the differential

$$
\phi_{*}: T M \rightarrow T B
$$

of $\phi$. Clearly, $T(M / B)$ is a subbundle of $T M$ which on each fibre $M_{b}=\phi^{-1}(b)$ restricts to be canonically isomorphic to $T M_{b}$. Let $g_{M / B}$ be a family of metrics on $M$, that is, $g_{M / B}$ is a metric on $T(M / B)$ which gives rise to a Riemannian metric on each fibre. Let $E$ be a complex vector bundle with an Euclidean metric $g^{E}$ and a connection $\nabla^{E}$.

This allows us to define a smooth family of Laplacians $\Delta_{M / B}$, which on a fibre $M_{b}=\phi^{-1}(b)$ acts as

$$
\Delta_{b} s=-\operatorname{Tr}\left(\nabla^{T^{*} M_{b} \otimes E} \nabla^{E} s\right), \quad s \in \mathcal{C}^{\infty}\left(M_{b} ; E_{b}\right), \quad E_{b}=\left.E\right|_{M_{b}},
$$

where $\nabla^{T^{*} M_{b} \otimes E}$ is induced from $\nabla^{E}$ and the Levi-Civita connection of $g_{b}=\left.g_{M / B}\right|_{M_{b}}$, and $\operatorname{Tr}(S) \in \mathcal{C}^{\infty}\left(M_{b} ; E_{b}\right)$ is the contraction of an element

$$
S \in \mathcal{C}^{\infty}\left(M_{b} ; T^{*} M_{b} \otimes T^{*} M_{b} \otimes E_{b}\right)
$$


with the dual metric $g_{b} \in \mathcal{C}^{\infty}\left(M_{b}, T M_{b} \otimes T M_{b}\right)$.

Let $\mathrm{L}^{2}(M / B ; E) \rightarrow B$ be the Hilbert bundle over $B$ with fibre at $b \in B$ given by $\mathrm{L}^{2}\left(M_{b} ; E_{b}\right)$. The scalar product on $\mathrm{L}^{2}\left(M_{b} ; E_{b}\right)$ is defined in the usual way by

$$
\left\langle s_{1}, s_{2}\right\rangle=\int g^{E}\left(s_{1}, \bar{s}_{2}\right) d g_{b}, \quad s_{1}, s_{2} \in \mathcal{C}^{\infty}\left(M_{b} ; E_{b}\right)
$$

where $d g_{b}$ is the volume form associated to the metric $g_{b}$.

As is well-known, the Laplacian $\Delta_{b}$ is self-adjoint and has a non-negative discrete spectrum. Moreover, eigensections with different eigenvalues are orthogonal in $\mathrm{L}^{2}\left(M_{b} ; E_{b}\right)$.

Definition 5.1. A spectral section $\left(\Pi_{M / B}, R_{1}, R_{2}\right)$ for the family of Laplacians $\Delta_{M / B}$ is a smooth family $\Pi_{M / B}: \mathrm{L}^{2}(M / B ; E) \rightarrow \mathrm{L}^{2}(M / B ; E)$ of projections

$$
\Pi_{b}: \mathrm{L}^{2}\left(M_{b} ; E_{b}\right) \rightarrow \mathrm{L}^{2}\left(M_{b} ; E_{b}\right), \quad b \in B
$$

with range a trivial vector bundle over $B$, together with real numbers $R_{1}$ and $R_{2}$, $R_{1}<R_{2}$, such that for all $b \in B$

$$
\Delta_{b} s=\lambda s \Longrightarrow \begin{cases}\Pi_{b} s=s & \text { if } \quad \lambda<R_{1} \\ \Pi_{b} s=0 & \text { if } \quad \lambda>R_{2}\end{cases}
$$

Remark 5.2. The triviality of the range of the spectral section is not in the original definition. We added this property because we will only consider spectral sections having as range a trivial vector bundle over B. Notice also that from the discreteness of the spectrum, this vector bundle must be of finite rank.

Obviously, one gets a spectral section by taking $\Pi_{M / B}=0$ and $R_{1}<R_{2}<0$, but this example is rather trivial. We would like to know about the existence of non-trivial spectral sections. The following result is due to R.B. Melrose.

Proposition 5.3. Let $\Delta_{M / B}$ be a family of Laplacians as in (5.1). Then given $R>0$, there exists $R^{\prime}>R$ and a smooth family of projections $\Pi_{M / B}$ such that $\left(\Pi_{M / B}, R, R^{\prime}\right)$ is a spectral section for $\Delta_{M / B}$.

Proof. By compactness, the discreteness of the spectrum and its continuity as a setvalued function, we can find a covering of $B$ by a finite number of open sets $\Omega_{i}$, and $R_{i} \in(R, \infty)$ such that $R_{i}$ is not in the spectrum of $\Delta_{b}$ for all $b \in \Omega_{i}$. The span of the eigensections with eigenvalues less than $R_{i}$ is then a smooth vector bundle $E_{i}$ over $\Omega_{i}$. Moreover, there are smooth bundle inclusions on all non-trivial intersections $\Omega_{i j}=\Omega_{i} \cap \Omega_{j}, I_{i j}: E_{i} \rightarrow E_{j}$, provided $R_{i} \leq R_{j}$. These inclusions satisfy obvious compatibility conditions on triple intersections.

By Kuiper's theorem (see [8]), the Hilbert bundle $\mathrm{L}^{2}(M / B ; E)$ is trivial in the uniform topology. It follows that there are trivial finite dimensional subbundles of arbitrary large rank. In fact, there is a sequence of families of finite rank projections 
$\pi_{b}^{(k)}$ with ranges trivial vector bundles over $B$ such that $\pi_{b}^{(k)} \rightarrow$ Id in the strong topology (on operators). Setting $\pi_{b}=\pi_{b}^{(k)}$ for sufficiently large $k$, it follows that the restriction of $\pi_{b}$ to the $E_{i}$ are injective

$$
\pi_{b}: E_{i, b} \hookrightarrow F_{b},
$$

where the trivial vector bundle $F$ is the range of $\pi$. By taking a larger $k$ if needed, we can also assume that for all $E_{i}$, the norm of $\left.\left(\operatorname{Id}-\pi_{b}\right)\right|_{E_{i}}$ is less than $\frac{1}{2}$. These embeddings of the bundles $E_{i}$ as subbundles of $\left.F\right|_{\Omega_{i}}$ are consistent with the inclusions $I_{i j}$. We may also consider the generalized inverse of $\pi_{b}$ on $E_{i}, m_{i}: F \rightarrow E_{i}$ over $\Omega_{i}$, defined as the composite of the orthogonal projection on $\pi\left(E_{i}\right)$ and the inverse of $\pi$ as a map from $E_{i}$ to $\pi\left(E_{i}\right)$.

Let $\mu_{i}$ be a partition of unity subordinate to the $\Omega_{i}$, and consider the family of linear maps

$$
f_{b}: F_{b} \rightarrow \mathrm{L}^{2}\left(M_{b} ; E_{b}\right), \quad f_{b}=\left(\mathrm{Id}-\pi_{b}\right) \sum_{j} \mu_{j}(b) m_{j}(b)
$$

This is a well-defined smooth family. Consider the open sets

$$
\mathcal{U}_{i}=\Omega_{i} \backslash \bigcup_{\left\{j ; R_{j}<R_{i}\right\}} \operatorname{supp}\left(\mu_{j}\right)
$$

These cover $B$. Over $\mathcal{U}_{i}$, the sum in (5.3) is limited to those $j$ with $\Omega_{i j} \neq \emptyset$ and $m_{j}\left(\pi_{b} e\right)=e$ for $e \in E_{i, b}$. Therefore, $f_{b}\left(\pi_{b} e\right)=\left(\operatorname{Id}-\pi_{b}\right) e$ for $b \in \mathcal{U}_{i}$ and $e \in E_{i, b}$. Since $\left\|f_{b}\right\|<\frac{1}{2}$, it follows that

$$
\left(\mathrm{Id}+f_{b}\right): F_{b} \rightarrow \mathrm{L}^{2}\left(M_{b} ; E_{b}\right)
$$

embeds $F$ as a subbundle of $\mathrm{L}^{2}(M / B ; E)$ which, over $\mathcal{U}_{i}$, contains $E_{i}$ as a subbundle.

Finally, choose $\psi \in \mathcal{C}^{\infty}(\mathbb{R})$ with $0 \leq \psi(t) \leq 1$ and such that

$$
\psi(t)= \begin{cases}1 & \text { if } \quad t<0 \\ 0 & \text { if } \quad t>1 .\end{cases}
$$

For $T \in \mathbb{R}$, consider the family of linear maps $Q_{T}=\psi\left(\Delta_{M / B}-T\right)$. For large $T$, $Q_{T} \circ($ Id $+f): F \rightarrow G$ embeds $F$ as a spectrally finite subbundle $G$ containing the $E_{i}$ over $\mathcal{U}_{i}$. Then, if $\Pi_{M / B}$ is the orthogonal projection on $G$ and $R^{\prime}=T+1$, we see that $\left(\Pi_{M / B}, R, R^{\prime}\right)$ is the desired spectral section. Since $F$ is a trivial vector bundle, it is clear that $G$ is a trivial vector bundle as well.

\subsection{Construction of the $K$-Class}

We are now ready to construct a $K$-class out of the indicial family of a Fredholm operator in $\mathcal{F}_{\Phi}^{-\infty}(X ; E)$. We need to distinguish two cases, the case where $\operatorname{dim} Z>0$ and the case where $\operatorname{dim} Z=0$. 


\subsubsection{The case where $\operatorname{dim} Z>0$}

Let

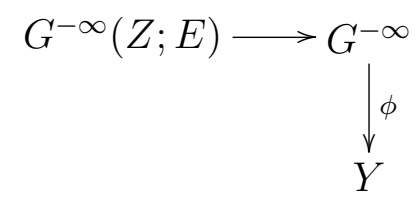

be the smooth bundle with fibre at $y \in Y$ given by $G^{-\infty}\left(Z_{y} ; E_{y}\right)$, where

$$
G^{-\infty}\left(Z_{y} ; E_{y}\right)=\left\{\operatorname{Id}+Q \mid Q \in \Psi^{-\infty}\left(Z_{y} ; E_{y}\right), \operatorname{Id}+Q \text { is invertible }\right\}
$$

is the group of invertible smoothing perturbations of the identity discussed in the introduction and $Z_{y}=\Phi^{-1}(y), E_{y}=\left.E\right|_{\Phi^{-1}(y)}$. The bundle $G^{-\infty}$ is a subbundle of the bundle $\mathcal{P}^{-\infty}$ in (3.10). Let $\pi: V \rightarrow Y$ be a real vector bundle over $\mathbb{R}$. In many of the situations interesting us, the vector bundle $V$ will be ${ }^{\Phi} N^{*} Y$. Let $\pi^{*} G^{-\infty}$ denote the pull-back of $G^{-\infty}$ on $V$.

Definition 5.4. A smooth section of $\pi^{*} G^{-\infty}$, or more generally of any bundle which has a section $\mathrm{Id}$, is said to be asymptotic to the identity if it converges rapidly with all derivatives to Id as one approaches infinity in $V$. Let $\Gamma_{\operatorname{Id}}\left(V ; \pi^{*} G^{-\infty}\right)$ denote the space of smooth sections of $\pi^{*} G^{-\infty}$ asymptotic to the identity.

The following corollary is an immediate consequence of proposition 3.14 and proposition 3.17.

Corollary 5.5. For $V={ }^{\Phi} N^{*} Y$, the indicial family $\mathrm{Id}+\widehat{A}_{0}$ of a Fredholm operator in $\mathcal{F}_{\Phi}^{-\infty}(X ; E)$ corresponds to a section of the bundle $\pi^{*} G^{-\infty} \rightarrow{ }^{\Phi} N^{*} Y$ which is asymptotic to the identity.

Our primary goal in this section is to construct a $K$-class out of the homotopy class of the indicial family of a Fredholm operator $(\operatorname{Id}+A) \in \mathcal{F}_{\Phi}^{-\infty}(X ; E)$. But considering an arbitrary real vector bundle $V$ over $Y$ will allow us to get a more general result which will turn out to be useful in chapter 7 . So we will consider the more general situation of a smooth section Id $+S$ of $\pi^{*} G^{-\infty} \rightarrow V$ asymptotic to the identity, bearing in mind that in the case where $V={ }^{\Phi} N^{*} Y$, the section $\operatorname{Id}+S$ can be seen as the indicial family of a Fredholm operator in $\mathcal{F}_{\Phi}^{-\infty}(X ; E)$.

We want to describe the homotopy classes of such sections. To this end, choose a family of metrics $g_{\partial X / Y}$ for the fibration $\Phi: \partial X \rightarrow Y$ and a Euclidean metric $g^{E}$ as well as a connection $\nabla^{E}$ for the bundle $E$. Then, as discussed in section 5.1 , there is an associated family of Laplacians $\Delta_{\partial X / Y}$. Given $y \in Y$, recall from section 2.3 that if $\left\{f_{i}\right\}_{i \in \mathbb{N}}$ is an orthonormal basis of eigensections of $\Delta_{y}$ with increasing eigenvalues, then

$$
\begin{aligned}
F_{i j}: \quad G^{-\infty}\left(Z_{y} ; E_{y}\right) & \rightarrow \mathcal{G}^{-\infty} \\
\operatorname{Id}+Q & \mapsto \delta_{i j}+\left\langle f_{i}, Q f_{j}\right\rangle_{\mathrm{L}^{2}}
\end{aligned}
$$

is an isomorphism between $G^{-\infty}\left(Z_{y} ; E_{y}\right)$ and the group $\mathcal{G}^{-\infty}$ of invertible semi-infinite 
matrices $\delta_{i j}+A_{i j}$ such that

$$
\sum_{i, j}(i+j)^{k}\left|A_{i j}\right|<\infty, \quad \forall k \in \mathbb{N}
$$

where $\delta_{i j}$ corresponds to the semi-infinite identity matrix. If $P_{y}(T)$ denotes the projection onto the span of the eigensections of $\Delta_{y}$ with eigenvalues less than $T$, this means that

$$
P_{y}(T)\left(Q_{y}\right) P_{y}(T) \underset{T \rightarrow+\infty}{\longrightarrow} Q
$$

in the $\mathcal{C}^{\infty}$-topology, so we can approximate $Q$ by spectrally finite smoothing operators.

Lemma 5.6. Let $(\operatorname{Id}+S)$ be a smooth section of $\pi^{*} G^{-\infty} \rightarrow V$ asymptotic to the identity, where $V \rightarrow Y$ is a real vector bundle over $Y$. Then there exists a spectral section $\left(\Pi, R_{1}, R_{2}\right)$ for $\Delta_{\partial X / Y}$ such that

$$
\left\|S_{b}-\Pi_{b} S_{b} \Pi_{b}\right\|<\frac{1}{2\left\|\left(\mathrm{Id}+S_{b}\right)^{-1}\right\|}, \quad \forall b \in V
$$

where $\|\cdot\|$ is the operator norm and $\Pi_{b}$ denotes $\Pi_{\pi(b)}$.

Proof. Using (5.8) and the fact $\mathrm{Id}+S$ is asymptotic to the identity, we see by the compactness of $Y$, the discreteness of the spectrum and its continuity as a set-valued function, that there exist a covering of $Y$ by a finite number of open sets $\Omega_{i}$ and numbers $T_{i} \in \mathbb{R}^{+}$such that

$$
\left\|S_{b}-P_{b}\left(T_{i}\right) S_{b} P_{b}\left(T_{i}\right)\right\|<\frac{1}{2\left\|\left(\operatorname{Id}+S_{b}\right)^{-1}\right\|}, \quad \forall b \in \pi^{*} \Omega_{i}
$$

where $P_{b}\left(T_{i}\right)$ denotes $P_{\pi(b)}\left(T_{i}\right)$. Set $R_{1}=\max _{i}\left\{T_{i}\right\}$. Then, by proposition 5.3 , there exists a smooth family of projections $\Pi$ and $R_{2}>R_{1}$ such that $\left(\Pi, R_{1}, R_{2}\right)$ is a spectral section for $\Delta_{\partial X / Y}$. By construction, we have that for $b \in \pi^{*} \Omega_{i}$,

$$
\Pi_{b} P_{b}\left(T_{i}\right) \Pi_{b}=P_{b}\left(T_{i}\right)
$$

so the estimate (5.9) also holds for $\Pi_{b}$ for all $b \in V$. Thus $\left(\Pi, R_{1}, R_{2}\right)$ is the desired spectral section.

Corollary 5.7. Let $\left(\Pi, R_{1}, R_{2}\right)$ be the spectral section of lemma 5.6. Then, $\mathrm{Id}+S$ and $\operatorname{Id}+\Pi S \Pi$ are homotopic as sections of $\pi^{*} G^{-\infty}$ asymptotic to the identity.

Proof. Consider the smooth homotopy

$$
t \mapsto \operatorname{Id}+S+t(\Pi S \Pi-S), \quad t \in[0,1] .
$$

For all $b \in V$ and $t \in[0,1]$, notice that

$$
\left(\mathrm{Id}+S_{b}\right)^{-1}\left(\mathrm{Id}+S_{b}+t\left(\Pi_{b} S_{b} \Pi_{b}-S_{b}\right)\right)=\operatorname{Id}+t\left(\operatorname{Id}+S_{b}\right)^{-1}\left(\Pi_{b} S_{b} \Pi_{b}-S_{b}\right)
$$


is invertible, since $\left\|t\left(\operatorname{Id}+S_{b}\right)^{-1}\left(\Pi_{b} S_{b} \Pi_{b}-S_{b}\right)\right\|<\frac{1}{2}$. This means that

$$
\mathrm{Id}+S_{b}+t\left(\Pi_{b} S_{b} \Pi_{b}-S_{b}\right)
$$

is invertible as well, and so the homotopy (5.10) between $(\operatorname{Id}+S)$ and $(\operatorname{Id}+\Pi S \Pi)$ is through sections of $\pi^{*} G^{-\infty}$ asymptotic to the identity.

Let $F$ denote the range of $\Pi$. Recall by our definition of a spectral section that $F$ is a trivial vector bundle of finite rank over $V$. Let $\mathrm{GL}(F, \mathbb{C}) \rightarrow{ }^{\Phi} N^{*} Y$ be the smooth bundle over $V$ with fibre at $b \in V$ given by $\operatorname{GL}\left(F_{\pi(b)}, \mathbb{C}\right)$, the group of complex linear isomorphism of $F_{\pi(b)}$. Then corollary 5.7 effectively reduces the section $\operatorname{Id}+S$ to a section

$$
\mathrm{Id}+S_{\Pi}=\Pi(\mathrm{Id}+S) \Pi: V \rightarrow \mathrm{GL}(F, \mathbb{C}) .
$$

If we also choose an explicit trivialization of $F$ and if $n$ is the rank of $F$, then the section Id $+S$ can be seen as smooth map

$$
\mathrm{Id}+S_{\Pi}: V \rightarrow \mathrm{GL}(n, \mathbb{C})
$$

asymptotic to the identity. Finally, since $\operatorname{GL}(n, \mathbb{C})$ is a topological subspace of the direct limit

$$
\mathrm{GL}(\infty, \mathbb{C})=\lim _{k \rightarrow \infty} \mathrm{GL}(k, \mathbb{C}),
$$

we can think of (5.12) as a map

$$
\mathrm{Id}+S_{\Pi}: V \rightarrow \mathrm{GL}(\infty, \mathbb{C})
$$

which converges to the identity at infinity. We are mostly interested in the homotopy class of this map.

Definition 5.8. Let $[V ; \mathrm{GL}(\infty, \mathbb{C})]$ denote the homotopy classes of continuous maps from $V$ to $\mathrm{GL}(\infty, \mathbb{C})$ which converges to the identity as one approaches infinity in $V$. If a is such a continuous map, then let [a] denotes its homotopy class in $[V ; \mathrm{GL}(\infty, \mathbb{C})]$.

Remark 5.9. The passage from the category of smooth maps to the category of continuous maps does not change the set of homotopy classes, see for instance proposition 17.8 in $[5]$.

Definition 5.10. If $(\mathrm{Id}+S)$ and $\left(\Pi, R_{1}, R_{2}\right)$ are as in corollary 5.7, then let $[\mathrm{Id}+S]_{\infty}$ denote the homotopy class of $(5.14)$ in $[V ; \mathrm{GL}(\infty, \mathbb{C})]$.

The homotopy class $[\mathrm{Id}+S]_{\infty}$ depends a priori on three choices:

1. The choice of an explicit trivialization of $F$,

2. The choice of a spectral section as in corollary 5.7,

3. The choice of a family of Laplacians $\Delta_{\partial X / Y}$. 
In the next three lemmas, we will show that in fact it is independent of these three choices.

Lemma 5.11. For $\Delta_{\partial X / Y}$ and $\left(\Pi, R_{1}, R_{2}\right)$ fixed, the homotopy class $[\operatorname{Id}+S]_{\infty}$ does not depend on the way $F$ is trivialized.

Proof. Suppose $\left(\mathrm{Id}+S_{\Pi}\right): V \rightarrow \mathrm{GL}(\infty, \mathbb{C})$ and $\left(\mathrm{Id}+S_{\Pi}^{\prime}\right): V \rightarrow \mathrm{GL}(\infty, \mathbb{C})$ arise from two different trivializations of $F$. Then,

$$
\mathrm{Id}+S_{\Pi}^{\prime}=M\left(\mathrm{Id}+S_{\Pi}\right) M^{-1}
$$

for some smooth map $M: V \rightarrow \mathrm{GL}(\infty, \mathbb{C})$ which is a pull-back of a map from $Y$ to $\mathrm{GL}(\infty, \mathbb{C})$. But at the homotopy level, the product $[a] \circ[b]=[a b]$ is commutative (see for instance the proof of proposition 2.12), so

$$
\begin{aligned}
{\left[\operatorname{Id}+S_{\Pi}^{\prime}\right] } & =\left[M\left(\operatorname{Id}+S_{\Pi}\right) M^{-1}\right]=[M] \circ\left[\operatorname{Id}+S_{\Pi}\right] \circ\left[M^{-1}\right] \\
& =\left[\operatorname{Id}+S_{\Pi}\right] \circ[M] \circ\left[M^{-1}\right]=\left[\operatorname{Id}+S_{\Pi}\right] \circ[\mathrm{Id}] \\
& =\left[\operatorname{Id}+S_{\Pi}\right] .
\end{aligned}
$$

Lemma 5.12. Assume the family of Laplacians $\Delta_{\partial X / Y}$ is fixed and let $(\mathrm{Id}+S)$ be as in lemma 5.6. If $\left(\Pi_{1}, R_{1}, T_{1}\right)$ and $\left(\Pi_{2}, R_{2}, T_{2}\right)$ are two spectral sections as in lemma 5.6, then $\mathrm{Id}+S_{\Pi_{1}}$ and $\mathrm{Id}+S_{\Pi_{2}}$ define the same homotopy class in $[V ; \mathrm{GL}(\infty, \mathbb{C})]$.

Proof. For $k \in \mathbb{N}$, set $R_{k}^{\prime}=\max \left\{T_{1}, T_{2}\right\}+k$. Then proposition 5.3 asserts the existence of a sequence of spectral sections $\left\{\left(\Pi_{k}^{\prime}, R_{k}^{\prime}, T_{k}^{\prime}\right)\right\}_{k \in \mathbb{N}}$ for the family of Laplacians $\Delta_{\partial X / Y}$ on $Y$. By construction,

$$
\Pi_{k}^{\prime} \Pi_{i} \Pi_{k}^{\prime}=\Pi_{i} \quad \text { for } i \in\{1,2\}, k \in \mathbb{N},
$$

so the estimate of lemma 5.6 applies as well to all the projections $\Pi_{k}^{\prime}$ :

$$
\left\|S_{b}-\Pi_{k, b}^{\prime} S_{b} \Pi_{k, b}^{\prime}\right\|<\frac{1}{2\left\|\left(\operatorname{Id}+S_{b}\right)^{-1}\right\|}, \quad \forall b \in V, \quad \forall k \in \mathbb{N} .
$$

Moreover, since $R_{k}^{\prime} \underset{k \rightarrow \infty}{\longrightarrow} \infty$, we know from (5.8), the fact that $\mathrm{Id}+S$ is asymptotic to the identity and the compactness of $Y \times[0,1]$ that in the $\mathcal{C}^{\infty}$-topology,

$$
\Pi_{k, b}^{\prime}\left(\mathrm{Id}+S_{b}+t\left(\Pi_{i, b} S_{b} \Pi_{i, b}-S_{b}\right)\right) \Pi_{k, b}^{\prime}+\mathrm{Id}-\Pi_{k, b}^{\prime} \underset{k \rightarrow \infty}{\longrightarrow} \operatorname{Id}+S_{b}+t\left(\Pi_{i, b} S_{b} \Pi_{i, b}-S_{b}\right)
$$

uniformly in $(b, t) \in V \times[0,1], i \in\{1,2\}$. By the proof of corollary 5.7, the limits of these two sequences are homotopies of sections of $\pi^{*} G^{-\infty}$ asymptotic to the identity. Thus, taking $k \in \mathbb{N}$ large enough, we can assume that

$$
t \mapsto \Pi_{k, b}^{\prime}\left(\mathrm{Id}+S_{b}+t\left(\Pi_{i, b} S_{b} \Pi_{i, b}-S_{b}\right)\right) \Pi_{k, b}^{\prime}+\mathrm{Id}-\Pi_{k, b}^{\prime}, \quad t \in[0,1],
$$


are homotopies of sections of $\pi^{*} G^{-\infty}$ asymptotic to the identity for $i \in\{1,2\}$. Then, if $F_{i}$ and $F_{k}^{\prime}$ denote the range of $\Pi_{i}$ and $\Pi_{k}^{\prime}$, we see that

$$
t \mapsto \Pi_{k}^{\prime}\left(\operatorname{Id}+S+t\left(\Pi_{i} S \Pi_{i}-S\right)\right) \Pi_{k}^{\prime}, \quad t \in[0,1],
$$

is a homotopy of sections of $\mathrm{GL}\left(F_{k}^{\prime}, \mathbb{C}\right)$ between $\Pi_{k}^{\prime}(\mathrm{Id}+S) \Pi_{k}^{\prime}$ and $\Pi_{k}^{\prime}\left(\mathrm{Id}+\Pi_{i} S \Pi_{i}\right) \Pi_{k}^{\prime}$ for $i \in\{1,2\}$. It is possible that the complements of the $F_{i}$ in $F_{k}^{\prime}$ are not trivial bundles, but by considering a spectral section $\left(\Pi_{j}, R_{j}^{\prime}, T_{j}^{\prime \prime}\right)$ with $R_{j}^{\prime}>T_{k}^{\prime}$ and $j$ large enough, we can add a trivial bundle to $F_{k}^{\prime}$ so that the complements of the $F_{i}$ become trivial $^{1}$ and so that $\left(\Pi_{k}, R_{k}, T_{j}^{\prime \prime}\right)$ is still a spectral section. So without loss of generality, we can assume that the complements of the $F_{i}$ in $F_{k}^{\prime}$ are trivial. Then, we see from lemma 5.11 that

$$
\begin{aligned}
{\left[\operatorname{Id}+S_{\Pi_{1}}\right] } & =\left[\operatorname{Id}+\left(\Pi_{1} S \Pi_{1}\right)_{\Pi_{k}^{\prime}}\right] \\
& =\left[\operatorname{Id}+S_{\Pi_{k}^{\prime}}\right] \quad \text { using }(5.15) \text { with } i=1, \\
& =\left[\operatorname{Id}+\left(\Pi_{2} S \Pi_{2}\right)_{\Pi_{k}^{\prime}}\right] \quad \text { using }(5.15) \text { with } i=2, \\
& =\left[\operatorname{Id}+S_{\Pi_{2}}\right] .
\end{aligned}
$$

Lemma 5.13. The homotopy class $[\mathrm{Id}+S]_{\infty}$ of definition 5.10 does not depend on the choice of the family of Laplacians $\Delta_{\partial X / Y}$.

Proof. Let $\Delta_{0}$ and $\Delta_{1}$ be two differents families of Laplacians for the fibration $\Phi$ : $\partial X \rightarrow Y$ and the complex vector bundle $E$, arising respectively from the metrics $g_{0}^{T(\partial X / Y)}, g_{0}^{E}$ and $g_{1}^{T(\partial X / Y)}, g_{1}^{E}$, and connections $\nabla_{0}^{E}$ and $\nabla_{1}^{E}$. We need to show that they lead to the same homotopy class in $[V ; \mathrm{GL}(\infty, \mathbb{C})]$.

By considering the homotopies of metrics

$$
t \mapsto g_{t}^{T(\partial X / Y)}=(1-t) g_{0}^{T(\partial X / Y)}+t g_{1}^{T(\partial X / Y)}, \quad t \in[0,1],
$$

and

$$
t \mapsto g_{t}^{E}=(1-t) g_{0}^{E}+t g_{1}^{E}, \quad t \in[0,1],
$$

and the homotopy of connections

$$
t \mapsto(1-t) \nabla_{0}^{E}+t \nabla_{1}^{E}, \quad t \in[0,1]
$$

one gets an associated homotopy of families of Laplacians

$$
t \mapsto \Delta_{t}, \quad t \in[0,1],
$$

where $\Delta_{t}$ is defined using the metrics $g_{t}^{T(\partial X / Y)}$ and $g_{t}^{E}$ and the connection $\nabla_{t}^{E}$. We can think of $\Delta_{t}$ as a family of Laplacians parametrized by $Y \times[0,1]$. A moment of reflection reveals that the proofs of lemma 5.6 and corollary 5.7 apply equally well if $Y$ is replaced by $Y \times[0,1]_{t}$ and $\operatorname{Id}+S$ is replaced by the section $\operatorname{Id}+p^{*} S$, where

\footnotetext{
${ }^{1}$ For instance, we can add a trivial bundle isomorphic to $F_{1} \oplus F_{2}$.
} 
$p: V \times[0,1]_{t} \rightarrow V$ is the projection on the left factor. This leads to a spectral section ( $\Pi, R_{1}, R_{2}$ ) satisfying the estimate of lemma 5.6 and such that

$$
t \mapsto \operatorname{Id}+p^{*} S+t\left(\Pi\left(p^{*} S\right) \Pi-p^{*} S\right), \quad t \in[0,1]
$$

is a homotopy of sections of $p^{*} \pi^{*} G^{-\infty}$ between $\left(\operatorname{Id}+p^{*} S\right)$ and $\left(\operatorname{Id}+\Pi\left(p^{*} S\right) \Pi\right)$. If $\Pi_{t}$ denotes the restriction of $\Pi$ to the slice $Y \times\{t\}$, then $\left(\Pi_{0}, R_{1}, R_{2}\right)$ and $\left(\Pi_{1}, R_{1}, R_{2}\right)$ are spectral sections for $\Delta_{0}$ and $\Delta_{1}$ which satisfy the conclusions of lemma 5.6 and corollary 5.7. Moreover, using lemma 5.11, we see from the homotopy

$$
t \mapsto \mathrm{Id}+\Pi_{t} S \Pi_{t}, \quad t \in[0,1],
$$

that $\left[\mathrm{Id}+S_{\Pi_{0}}\right]_{\infty}=\left[\mathrm{Id}+S_{\Pi_{1}}\right]_{\infty}$. From lemma 5.12, we conclude that the homotopy class of definition 5.10 is the same for $\Delta_{0}$ and $\Delta_{1}$.

From lemmas 5.11, 5.12 and 5.13, we get the following

Proposition 5.14. The homotopy class $[\mathrm{Id}+S]_{\infty}$ of definition 5.10 is well-defined, that is, it is independent of the three choices involved in its construction.

Definition 5.15. Let $\left[V ; \pi^{*} G^{-\infty}\right]$ denote the set of homotopy classes of smooth sections of $\pi^{*} G^{-\infty}$ asymptotic to the identity. If $\mathrm{Id}+S$ is a section of $\pi^{*} G^{-\infty}$ asymptotic to the identity, let $[\mathrm{Id}+S]$ denotes its homotopy class in $\left[V ; \pi^{*} G^{-\infty}\right]$.

Lemma 5.16. If $\mathrm{Id}+S$ is a section of $\pi^{*} G^{-\infty}$ asymptotic to the identity, then $[\mathrm{Id}+S]_{\infty} \in[V ; \mathrm{GL}(\infty, \mathbb{C})]$ only depends on the homotopy class of $\operatorname{Id}+S$ in the set $\left[V ; \pi^{*} G^{-\infty}\right]$.

Proof. Assume that $t \mapsto \mathrm{Id}+S_{t}, t \in[0,1]$, is a homotopy of sections of $\pi^{*} G^{-\infty}$ asymptotic to the identity. We need to show that $\left[\mathrm{Id}+S_{0}\right]_{\infty}=\left[\mathrm{Id}+S_{1}\right]_{\infty}$. Since $[0,1]$ is compact, a moment of thought reveals that the proofs of lemma 5.6 and corollary 5.7 work equally well if one replaces $V$ by the bundle $[0,1] \times V \rightarrow Y$. What one gets is that there exists a spectral section $\left(\Pi, R_{1}, R_{2}\right)$ for $\Delta_{\partial X / Y}$ on $Y$ so that

$$
\left\|S_{t}(b)-\Pi_{b} S_{t}(b) \Pi_{b}\right\|<\frac{1}{2\left\|\left(\operatorname{Id}+S_{t}(b)\right)^{-1}\right\|}, \quad \forall b \in V, t \in[0,1],
$$

which implies that $\mathrm{Id}+S_{t}$ and $\mathrm{Id}+\Pi S_{t} \Pi$ are homotopic as smooth homotopies of smooth sections of $\pi^{*} G^{-\infty}$ asymptotic to the identity. In particular, after trivializing the range of $\Pi, \mathrm{Id}+\Pi S_{0} \Pi$ and $\mathrm{Id}+\Pi S_{1} \Pi$ define the same homotopy class in $[V ; \mathrm{GL}(\infty, \mathbb{C})]$, and we conclude that

$$
\left[\operatorname{Id}+S_{0}\right]_{\infty}=\left[\operatorname{Id}+S_{1}\right]_{\infty}
$$

Thus, we see that definition 5.10 gives us a canonical map

$$
\begin{aligned}
I_{\infty}:\left[V ; \pi^{*} G^{-\infty}\right] & \rightarrow[V ; \operatorname{GL}(\infty, \mathbb{C})] \\
{[\operatorname{Id}+S] } & \mapsto[\operatorname{Id}+S]_{\infty} .
\end{aligned}
$$


Proposition 5.17. The canonical map $I_{\infty}$ is an isomorphism of sets.

Proof. The proof of the surjectivity of $I_{\infty}$ reduces to the existence of a spectral section with range a vector bundle of arbitrary large rank. But the existence of such a spectral section is an easy consequence of proposition 5.3 together with the compactness of $Y$ and the continuity of the spectrum as a set-valued function.

For the proof of the injectivity of $I_{\infty}$, suppose $\left[\mathrm{Id}+S_{0}\right]_{\infty}=\left[\mathrm{Id}+S_{1}\right]_{\infty}$. Let $\Pi_{0}$ and $\Pi_{1}$ be spectral sections as in lemma 5.6 that can be used to define $\left[\operatorname{Id}+S_{0}\right]_{\infty}$ and $\left[\mathrm{Id}+S_{1}\right]_{\infty}$ respectively. Let $\Pi$ be another spectral section such that $\Pi_{i} \Pi=\Pi_{i}$ for $i \in\{0,1\}$ (cf. proof of lemma 5.12). Then $\Pi$ can also be used to define $\left[\mathrm{Id}+S_{0}\right]_{\infty}$ and $\left[\operatorname{Id}+S_{1}\right]_{\infty}$. Taking $\Pi$ to have a larger rank if necessary, we can assume that there is a homotopy

$$
t \mapsto \mathrm{Id}+\Pi S_{t} \Pi, \quad t \in[0,1],
$$

through sections of $\pi^{*} G^{-\infty}$ asymptotic to the identity. From corollary 5.7, we then deduce that $\left[\operatorname{Id}+S_{0}\right]=\left[\operatorname{Id}+S_{1}\right]$ in $\left[V ; \pi^{*} G^{-\infty}\right]$. This shows that the map $I_{\infty}$ is injective.

Before discussing the associated $K$-class, let us concentrate on the second case.

\subsubsection{The case where $\operatorname{dim} Z=0$}

The case where $\operatorname{dim} Z=0$ turns out to be significantly easier. This is somehow the reverse procedure of the case where $\operatorname{dim} Z>0$, that is, instead of reducing an infinite bundle to a trivial vector bundle, we will enlarge a finite vector bundle to make it trivial. The case $\operatorname{dim} Z=0$ evidently includes scattering operators, but it means more generally that the fibration $\Phi: \partial X \rightarrow Y$ is a finite covering.

Definition 5.18. Let $\mathcal{E} \rightarrow Y$ be the complex vector bundle on $Y$ with fibre at $y \in Y$ given by

$$
\mathcal{E}_{y}=\bigoplus_{z \in \Phi^{-1}(y)} E_{y}
$$

Definition 5.19. Let $\operatorname{GL}(\mathcal{E}, \mathbb{C})$ be the bundle over $Y$ with fibre at $y \in Y$ given by $\operatorname{GL}\left(\mathcal{E}_{y}, \mathbb{C}\right)$. Let $\pi^{*} \mathrm{GL}(\mathcal{E}, \mathbb{C}) \rightarrow{ }^{\Phi} N^{*} Y$ be the pull-back of $\operatorname{GL}(\mathcal{E}, \mathbb{C})$ on ${ }^{\Phi} N^{*} Y$.

An immediate consequence of remark 3.16 and proposition 3.14 is the following

Corollary 5.20. The indicial family $\left(\mathrm{Id}+\widehat{A}_{0}\right)$ of a Fredholm operator $(\operatorname{Id}+A) \in$ $\mathcal{F}_{\Phi}^{-\infty}(X ; E)$ is a section of the bundle $\pi^{*} \mathrm{GL}(\mathcal{E} ; \mathbb{C})$ which is asymptotic to the identity.

Let $\mathcal{F} \rightarrow Y$ be a complex vector bundle over $Y$ such that $\mathcal{E} \oplus \mathcal{F}$ is trivial. Such a bundle always exists, see for instance corollary 1.4.14 in [1]. If $n$ is the rank of $\mathcal{E} \oplus \mathcal{F}$, 
so that $\mathcal{E} \oplus \mathcal{F} \cong \underline{\mathbb{C}}^{n}$, then

$$
\begin{aligned}
\pi^{*} \mathrm{GL}(\mathcal{E}, \mathbb{C}) & \subset \pi^{*} \mathrm{GL}(\mathcal{E} \oplus \mathcal{F}, \mathbb{C}) \\
& \cong \pi^{*} \mathrm{GL}\left(\underline{\mathbb{C}}^{n}, \mathbb{C}\right) \\
& =\mathrm{GL}(n, \mathbb{C}) \times{ }^{\Phi} N^{*} Y \\
& \subset \mathrm{GL}(\infty, \mathbb{C}) \times{ }^{\Phi} N^{*} Y
\end{aligned}
$$

Definition 5.21. If $(\mathrm{Id}+S)$ is the indicial family of some operator in $\mathcal{F}_{\Phi}^{-\infty}(X ; E)$, let $\left(\mathrm{Id}+S_{\mathcal{F}}\right):{ }^{\Phi} N^{*} Y \rightarrow \mathrm{GL}(\infty ; \mathbb{C})$ be the associated map under the series of inclusions (5.17) and let $[\mathrm{Id}+S]_{\infty} \in\left[{ }^{\Phi} N^{*} Y ; \mathrm{GL}(\infty ; \mathbb{C})\right]$ denotes its homotopy class.

As in the previous case, we want to show that the homotopy class $[\operatorname{Id}+S]_{\infty}$ does not depend on the choices made to define it, namely the choice of an explicit trivialization of $\mathcal{E} \oplus \mathcal{F}$ and the choice of $\mathcal{F}$.

Proposition 5.22. The homotopy class $[\mathrm{Id}+S]_{\infty}$ of definition 5.21 is well-defined, that is, it does not depend on the choice of $\mathcal{F}$ and on the way $\mathcal{E} \oplus \mathcal{F}$ is trivialized.

Proof. First, the homotopy class $[\operatorname{Id}+S]_{\infty}$ does not depend on the way $\mathcal{E} \oplus \mathcal{F}$ is trivialized. It is the same proof as lemma 5.11.

Secondly, the homotopy class $[\mathrm{Id}+S]_{\infty}$ does not depend on the choice of the vector bundle $\mathcal{F}$ such that $\mathcal{E} \oplus \mathcal{F}$ is trivial.

Indeed, let $\mathcal{G} \rightarrow Y$ be another complex vector bundle such that $\mathcal{E} \oplus \mathcal{G}$ is trivial. Then consider the trivial vector bundle $\mathcal{H}=\mathcal{E} \oplus F \oplus \mathcal{E}^{\prime} \oplus \mathcal{G}$, where $\mathcal{E}^{\prime} \cong \mathcal{E}$. The bundles $\mathcal{E} \oplus \mathcal{F}$ and $\mathcal{E} \oplus \mathcal{G}$ are subbundles of $\mathcal{H}$, and to be more precise, we consider the inclusions

$$
\begin{array}{rlrl}
i_{1}: \mathcal{E} \oplus \mathcal{F} & \hookrightarrow \mathcal{E} \oplus F \oplus \mathcal{E}^{\prime} \oplus \mathcal{G}, \quad i_{2}: \mathcal{E} \oplus \mathcal{G} & \hookrightarrow \mathcal{E} \oplus F \oplus \mathcal{E}^{\prime} \oplus \mathcal{G} \\
(e, f) & \mapsto(e, f, 0,0) & (e, g) & \mapsto(e, 0,0, g)
\end{array}
$$

Clearly, the complements of $\mathcal{E} \oplus \mathcal{F}$ and $\mathcal{E} \oplus \mathcal{G}$ in $\mathcal{H}$ are trivial vector bundles, so this means that $\mathcal{F}$ and $\mathcal{F} \oplus \mathcal{E}^{\prime} \oplus \mathcal{G}$ lead to the same homotopy class, and similarly for $\mathcal{G}$ and $\mathcal{F} \oplus \mathcal{E}^{\prime} \oplus \mathcal{G}$. In particular $\mathcal{F}$ and $\mathcal{G}$ lead to same homotopy class.

In this case, it is straightforward to see that the homotopy class $[\operatorname{Id}+S]_{\infty}$ only depends on the homotopy class of $\operatorname{Id}+S$ in $\left[{ }^{\Phi} N^{*} Y ; \pi^{*} \mathrm{GL}(\mathcal{E}, \mathbb{C})\right]$. So definition 5.21 gives also rise to a canonical map

$$
\begin{aligned}
I_{\infty}:\left[{ }^{\Phi} N^{*} Y ; \pi^{*} \mathrm{GL}(\mathcal{E}, \mathbb{C})\right] & \rightarrow\left[{ }^{\Phi} N^{*} Y ; \mathrm{GL}(\infty, \mathbb{C})\right] \\
{[\operatorname{Id}+S] } & \mapsto[\operatorname{Id}+S]_{\infty} .
\end{aligned}
$$

However, it is not an isomorphism in general, but all classes arise this way if arbitrary bundles are admitted. 


\subsubsection{The associated $K$-class}

Now that in both cases $\operatorname{dim} Z>0$ and $\operatorname{dim} Z=0$ we can associate a well-defined homotopy class

$$
\left[\mathrm{Id}+\widehat{A}_{0}\right]_{\infty} \in\left[{ }^{\Phi} N^{*} Y ; \mathrm{GL}(\infty ; \mathbb{C})\right]
$$

to the indicial family $\left(\operatorname{Id}+\widehat{A}_{0}\right)$ of a Fredholm operator $(\operatorname{Id}+A)$ in $\mathcal{F}_{\Phi}^{-\infty}(X ; E)$, let us try to reinterpret this homotopy class in terms of $K$-theory. We will follow the notation of [1]. Recall from corollary 2.13 that the space $\mathrm{GL}(\infty, \mathbb{C})$ is a classifying space for odd $K$-theory, so we have the correspondence

$$
\left[{ }^{\Phi} N^{*} Y ; \mathrm{GL}(\infty, \mathbb{C})\right] \cong \widetilde{K}^{0}\left(\mathbb{S}^{1} \wedge Y^{\Phi} N^{*} Y\right),
$$

where $Y^{\Phi} N^{*} Y$ is the Thom space of ${ }^{\Phi} N^{*} Y$, in other words its one point compactification, and

$$
\mathbb{S}^{1} \wedge Y^{\Phi} N^{*} Y=\left(\mathbb{S}^{1} \times Y^{\Phi} N^{*} Y\right) /\left(\mathbb{S}^{1} \times\{\infty\} \cup\{1\} \times Y^{\Phi} N^{*} Y\right)
$$

is the reduced suspension of $Y^{\Phi} N^{*} Y, \mathbb{S}^{1} \subset \mathbb{C}$ being the unit circle. Since ${ }^{\Phi} N^{*} Y \cong$ $T^{*} Y \times \mathbb{R}$, we see that

$$
Y^{\Phi} N^{*} Y \cong \mathbb{S}^{1} \wedge Y^{T^{*} Y}=\left(\mathbb{S}^{1} \times Y^{T^{*} Y}\right) /\left(\mathbb{S}^{1} \times\{\infty\} \cup\{1\} \times Y^{T^{*} Y}\right),
$$

the factor " $\mathbb{R}$ " being effectively turned into a suspension. This means that

$$
\begin{aligned}
{\left[{ }^{\Phi} N^{*} Y ; \mathrm{GL}(\infty, \mathbb{C})\right] } & \cong \widetilde{K}^{0}\left(\mathbb{S}^{1} \wedge \mathbb{S}^{1} \wedge Y^{T^{*} Y}\right) \\
& =\widetilde{K}^{-2}\left(Y^{T^{*} Y}\right) \\
& \cong \widetilde{K}^{0}\left(Y^{T^{*} Y}\right) \text { by the periodicity theorem } \\
& =K_{c}^{0}\left(T^{*} Y\right),
\end{aligned}
$$

where $K_{c}^{0}\left(T^{*} Y\right)$, the $K$-theory with compact support of $T^{*} Y$, is by definition equal to $\widetilde{K}^{0}\left(Y^{T^{*} Y}\right)$. Thus, we see from $(5.19)$ that the homotopy class $\left[\mathrm{Id}+\widehat{A}_{0}\right]$ in $\left[{ }^{\Phi} N^{*} Y ; \mathrm{GL}(\infty, \mathbb{C})\right]$ defines a $K$-class in $K_{c}^{0}\left(T^{*} Y\right)$.

Definition 5.23. If $\left(\mathrm{Id}+\widehat{A}_{0}\right)$ is the indicial family of a Fredholm operator $(\mathrm{Id}+A)$ in $\mathcal{F}_{\Phi}^{-\infty}(X ; E)$, let $\kappa(\operatorname{Id}+A) \in K_{c}^{0}\left(T^{*} Y\right)$ denote the $K$-class associated to the homotopy class $\left[\mathrm{Id}+\widehat{A}_{0}\right]_{\infty}$ under the identification $\left[{ }^{\Phi} N^{*} Y ; \mathrm{GL}(\infty, \mathbb{C})\right] \cong K_{c}^{0}\left(T^{*} Y\right)$ given by (5.19). 


\section{Chapter 6}

\section{The Index in terms of $K$-theory}

Having associated a $K$-class $\kappa(\operatorname{Id}+A) \in K_{c}^{0}\left(T^{*} Y\right)$ to a Fredholm operator $(\mathrm{Id}+A)$ in $\mathcal{F}_{\Phi}^{-\infty}(X ; E)$, the obvious guess is that the index of $\mathrm{Id}+A$ is given by

$$
\operatorname{ind}(\operatorname{Id}+A)=\operatorname{ind}_{t}(\kappa(\operatorname{Id}+A))
$$

where

$$
\operatorname{ind}_{t}: K_{c}^{0}\left(T^{*} Y\right) \rightarrow \mathbb{Z}
$$

is the topological index introduced by Atiyah and Singer in [2]. This is indeed the case. We will prove (6.1) by reducing the computation of the index to the case of a scattering operator.

\subsection{Reduction to the case of a scattering operator}

To the manifold $X$ with fibred cusp structure given by the fibration $\Phi: \partial X \rightarrow Y$ and some defining function $x$ of $\partial X$, let us associate the manifold with boundaries

$$
\mathcal{W}_{Y}=[0,1]_{t} \times Y, \quad \partial \mathcal{W}_{Y}=Y_{0} \cup Y_{1},
$$

where $Y_{0}=\{0\} \times Y \subset \mathcal{W}_{Y}$ and $Y_{1}=\{1\} \times Y \subset \mathcal{W}_{Y}$. The boundary $\partial \mathcal{W}_{Y}$ has an obvious defining function given by $t$ near $Y_{0}$ and by $(1-t)$ near $Y_{1}$, where $t \in[0,1]$ is the variable of the left factor of $[0,1]_{t} \times Y$. It will have a fibred cusp structure if one considers the trivial fibration given by the identity

$$
\text { Id }: \partial \mathcal{W}_{Y} \rightarrow \partial \mathcal{W}_{Y}
$$

If $F \rightarrow \mathcal{W}_{Y}$ is a complex vector bundle on $\mathcal{W}_{Y}$, then we can define the algebra of fibred cusp operators $\Psi_{\text {Id }}^{*}\left(\mathcal{W}_{Y} ; F\right)$ acting on sections of $F$. From the previous section, we know that a Fredholm operator in $\mathcal{F}_{\text {Id }}^{-\infty}\left(\mathcal{W}_{Y} ; F\right)$ gives rise to a well define element in

$$
K_{c}^{0}\left(T^{*}\left(\partial \mathcal{W}_{Y}\right)\right) \cong K_{c}^{0}\left(T^{*} Y_{0}\right) \oplus K_{c}^{0}\left(T^{*} Y_{1}\right)
$$

Definition 6.1. Let $i_{1}: K_{c}^{0}\left(T^{*} Y\right) \hookrightarrow K_{c}^{0}\left(T^{*}\left(\partial \mathcal{W}_{Y}\right)\right)$ be the natural inclusion given 
by

$$
\begin{aligned}
i_{1}: \quad K_{c}^{0}\left(T^{*} Y\right) & \hookrightarrow K_{c}^{0}\left(T^{*} Y_{0}\right) \oplus K_{c}^{0}\left(T^{*} Y_{1}\right) \\
a & \mapsto(0, a) .
\end{aligned}
$$

Lemma 6.2. If $(\operatorname{Id}+A) \in \mathcal{F}_{\Phi}^{-\infty}(X ; E)$ is such that the indicial family $\widehat{A}_{0}$, seen as a section of $\pi^{*} \mathcal{P}^{-\infty}$, lies in some subbundle $\pi^{*} \operatorname{End}(F) \subset \pi^{*} \mathcal{P}^{-\infty}$, where $F \rightarrow Y$ is some trivial finite rank compex vector bundle, then any scattering operator $(\operatorname{Id}+S) \in$ $\mathcal{F}_{\text {Id }}^{-\infty}\left(\mathcal{W}_{Y} ; F\right)$ such that

$$
\kappa(\operatorname{Id}+S)=i_{1} \kappa(\operatorname{Id}+A), \text { in } K_{c}^{0}\left(T^{*} \mathcal{W}_{Y}\right)
$$

has the same index as $\operatorname{Id}+A$.

Proof. By proposition 4.8, we can assume that $N_{\Phi}^{\prime}(A)=A_{0}$, that is, $\widehat{A}_{k}=0$ for $k \in \mathbb{N}$. In fact, using some cut-off function $\chi\left(x, x^{\prime}\right)$ supported in $x<\epsilon, x^{\prime}<\epsilon$ with $\chi \equiv 1$ near $x=x^{\prime}=0$, we can assume that the Schwartz kernel $K_{A}$ of $A$ is of the form

$$
K_{A}\left(x, y, z, S, Y, z-z^{\prime}\right)=\chi\left(x, x^{\prime}\right) K_{A_{0}}\left(y, z, S, Y, z-z^{\prime}\right)
$$

in the local coordinates (3.7). Thus, the support of $K_{A}$ is arbitrary closed to the front face $\mathrm{ff}_{\Phi} \subset X_{\Phi}^{2}$. This means that Id $+A$ acts as the identity outside the collar neighborhood $\partial X \times[0, \epsilon)_{x} \subset X$ of the boundary $\partial X$. Moreover, by the assumption on $\widehat{A}_{0}$, the operator Id $+A$ has a well-defined action on $\mathcal{C}_{c}^{\infty}\left(Y \times[0, \epsilon)_{x} ; F\right)$ and

$$
\operatorname{ker}(\operatorname{Id}+A) \subset \mathcal{C}_{c}^{\infty}\left(Y \times[0, \epsilon)_{x} ; F\right), \quad \operatorname{coker}(\operatorname{Id}+A) \subset \mathcal{C}_{c}^{\infty}\left(Y \times[0, \epsilon)_{x} ; F\right),
$$

where we define the cokernel of $\operatorname{Id}+A$ to be the orthogonal complement of the range of $\mathrm{Id}+A$. Under the embedding

$$
\begin{aligned}
i: Y \times[0, \epsilon)_{x} & \hookrightarrow \mathcal{W}_{Y} \\
(y, x) & \mapsto(y, 1-x),
\end{aligned}
$$

this action extends to give an operator $\operatorname{Id}+S \in \mathcal{F}_{\text {Id }}^{-\infty}\left(\mathcal{W}_{Y} ; F\right)$ such that

$$
\kappa(\operatorname{Id}+S)=i_{1} \kappa(\operatorname{Id}+A), \text { in } K_{c}^{0}\left(T^{*} \mathcal{W}_{Y}\right) .
$$

The kernel and cokernel of Id $+S$ are canonically identified with the kernel and cokernel of $\mathrm{Id}+A$, so in particular $\mathrm{Id}+S$ has the same index as $\mathrm{Id}+A$.

Lemma 6.3. Let $(\operatorname{Id}+A) \in \mathcal{F}_{\Phi}^{-\infty}(X ; E)$ be a Fredholm operator and let $\kappa(\operatorname{Id}+A)$ be its associated $K$-class in $K_{c}^{0}\left(T^{*} Y\right)$. Then, for $F$ a trivial vector bundle with sufficiently large rank, there exists a scattering operator $\operatorname{Id}+S \in \mathcal{F}_{\mathrm{Id}}^{-\infty}\left(\mathcal{W}_{Y} ; F\right)$ with associated $K$-class given by $i_{1} \kappa(\operatorname{Id}+A) \in K_{c}^{0}\left(T^{*}\left(\partial \mathcal{W}_{Y}\right)\right)$ and such that $\operatorname{ind}(\operatorname{Id}+A)=$ ind $(\mathrm{Id}+S)$.

Proof. The proof relies on lemma 6.2, but is slightly different depending on whether or $\operatorname{not} \operatorname{dim} Z>0$. 
First assume that $\operatorname{dim} Z>0$. Then let $\left(\Pi, R_{1}, R_{2}\right)$ be a spectral section as in lemma 5.6 for the indicial family $\mathrm{Id}+\widehat{A}_{0}$. By corollary $5.7,\left(\mathrm{Id}+\Pi \widehat{A}_{0} \Pi\right)$ is homotopic to $\left(\mathrm{Id}+\widehat{A}_{0}\right)$. Let $P \in \Psi_{\Phi}^{-\infty}(X ; E)$ be such that $\widehat{P}_{0}=\Pi \widehat{A}_{0} \Pi$. Then by proposition 3.14 and proposition $4.8,(\operatorname{Id}+P) \in \mathcal{F}_{\Phi}^{-\infty}(X ; E)$ has the same index as $\operatorname{Id}+A$ and it defines the same $K$-class in $K_{c}^{0}\left(T^{*} Y\right)$. By taking $F \rightarrow Y$ to be the range of $\Pi$ on $Y$, we can then apply lemma 6.2 to $\mathrm{Id}+P$. Thus there exists $(\mathrm{Id}+S) \in \mathcal{F}_{\mathrm{Id}}^{-\infty}\left(\mathcal{W}_{Y} ; F\right)$ such that $\operatorname{ind}(\operatorname{Id}+S)=\operatorname{ind}(\operatorname{Id}+P)$ and $\kappa(\operatorname{Id}+S)=i_{1} \kappa(\operatorname{Id}+P)$. So $\operatorname{Id}+S$ is the desired operator.

If $\operatorname{dim} Z=0$ let $\mathcal{E} \rightarrow Y$ be as in definition 5.18. From lemma 6.2, there exists $(\operatorname{Id}+S) \in \mathcal{F}_{\text {Id }}^{-\infty}\left(\mathcal{W}_{Y} ; \mathcal{E}\right)$ having the same index as $\operatorname{Id}+A$ and having $i_{1} \kappa(\operatorname{Id}+A)$ as an associated $K$-class. If $\mathcal{E}$ is not trivial, let $\mathcal{G} \rightarrow \mathcal{W}_{Y}$ be another complex vector bundle such that $F=\mathcal{E} \oplus \mathcal{G}$ is trivial and let $\operatorname{Id}+S^{\prime} \in \mathcal{F}_{\text {Id }}^{-\infty}\left(\mathcal{W}_{Y} ; F\right)$ be the operator which acts as $(\mathrm{Id}+S)$ on sections of $\mathcal{E}$ and as the identity on sections of $\mathcal{G}$. Clearly, $\mathrm{Id}+S^{\prime}$ has the same index as Id $+S$ and they define the same $K$-class. So $\mathrm{Id}+S^{\prime}$ is the desired operator.

\subsection{Computation of the index}

This reduces the problem to the computation of the index of a scattering operator. In section 6.5 of [16], a general topological formula for the index of scattering operators is derived. For the convenience of the reader, we will adapt the discussion that can be found there to our particular context.

Given $(\operatorname{Id}+S) \in \mathcal{F}_{\text {Id }}^{-\infty}\left(\mathcal{W}_{Y} ; F\right)$ as in lemma 6.3 with $F=\underline{\mathbb{C}}^{n}$, the indicial family Id $+\widehat{S}_{0}$ of Id $+S$ can be seen as a map

$$
\mathrm{Id}+\widehat{S}_{0}:{ }^{\mathrm{Id}} N^{*} \partial \mathcal{W}_{Y} \rightarrow \operatorname{GL}(n, \mathbb{C}) .
$$

The homotopy class of this map gives rise to the associated $K$-class in $K_{c}^{0}\left(T^{*} \partial \mathcal{W}_{Y}\right)$ under the identification (5.19). Let $\mathcal{V}_{Y}$ be the double of $\mathcal{W}_{Y}$ obtained by taking two copies $\mathcal{W}_{Y}$ and $\mathcal{W}_{Y}^{\prime}$ of $\mathcal{W}_{Y}$ and identifying $Y_{i}$ of the first copy with $Y_{i}^{\prime}$ of the second copy for $i \in\{0,1\}$. Thus, $\mathcal{V}_{Y} \cong \mathbb{S}^{1} \times Y$.

Identifying the tangent bundle with the cotangent bundle via some metric, we see that inside $T^{*} \mathcal{V}_{Y},{ }^{\text {Id }} N^{*} \partial \mathcal{W}_{Y}$ naturally identifies with $\left.T^{*} \mathcal{W}_{Y}\right|_{\partial \mathcal{W}_{Y}}$. Via the clutching construction (see proposition 2.12), the map (6.3) defines a $K$-class with compact support in $K_{c}^{0}\left(T^{*} \mathcal{V}_{Y}\right)$. More generally,

Definition 6.4. For $(\operatorname{Id}+S) \in \mathcal{F}_{\mathrm{Id}}^{-\infty}\left(\mathcal{W}_{Y} ; \underline{\mathbb{C}}^{n}\right)$, let $c(\operatorname{Id}+S) \in K_{c}^{0}\left(T^{*} \mathcal{V}_{Y}\right)$ be the element defined by applying the clutching construction to the map (6.3).

The following proposition is a particular case of theorem 6.4 in [16].

Proposition 6.5. The index of $(\operatorname{Id}+S) \in \mathcal{F}_{\mathrm{Id}}^{-\infty}\left(\mathcal{W}_{Y} ; \underline{\mathbb{C}}^{n}\right)$ is given by

$$
\operatorname{ind}(\operatorname{Id}+S)=\operatorname{ind}_{t}(c(\operatorname{Id}+S))
$$


where $\operatorname{ind}_{t}: K_{c}^{0}\left(T^{*} \mathcal{V}_{Y}\right) \rightarrow \mathbb{Z}$ is the topological index map.

Proof. Let us enlarge our space of operators and see $\mathrm{Id}+S$ as a Fredholm operator in $\Psi_{\text {Id }}^{0}\left(\mathcal{W}_{Y} ; \mathbb{C}^{n}\right)$. Then proposition 3.14 can be reinterpreted as saying that the $K$ class defined by the symbol $\sigma_{0}(\operatorname{Id}+S)$ is null when restricted to $\left.T^{*} \mathcal{W}_{Y}\right|_{\partial \mathcal{W}_{Y}}$. This means one can smoothly deform Id $+S$ through Fredholm operators in $\Psi_{\text {Id }}^{0}\left(\mathcal{W}_{Y} ; \underline{\mathbb{C}}^{n}\right)$ to a Fredholm operator $P \in \Psi_{\text {Id }}^{0}\left(\mathcal{W}_{Y} ; \underline{\mathbb{C}}^{n}\right)$ that acts by multiplication of a matrix near $\partial \mathcal{W}_{Y}$. This new operator is no longer in $\mathcal{F}_{\text {Id }}^{-\infty}\left(\mathcal{W}_{Y} ; \underline{\mathbb{C}}^{n}\right)$ in general, but it has the same index as Id $+S$. A general Fredholm operator $Q \in \Psi_{\mathrm{Id}}^{0}\left(\mathcal{W}_{Y} ; \underline{\mathbb{C}}^{n}\right)$ also defines an elment of $K_{c}^{0}\left(T^{*} \mathcal{V}_{Y}\right)$ but one needs also the symbol. If $\overline{T^{*} \mathcal{W}_{Y}}$ is the radial compactification of $T^{*} \mathcal{W}_{Y}$, then the indicial family $\widehat{Q}_{0}$ together with the symbol $\sigma_{0}$ form a continuous map

$$
f: \partial\left(\overline{T^{*} \mathcal{W}_{Y}}\right) \rightarrow G L(n, \mathbb{C}) .
$$

This map can be be used to defined a relative $K$-class on the double $D_{\overline{T^{*} \mathcal{W}_{Y}}}$ of $\overline{T^{*} \mathcal{W}_{Y}}$ obtained by identifying two copies of $\overline{T^{*} \mathcal{W}_{Y}}$ at their boundaries. This class will in fact have support inside $T^{*} \mathcal{V}_{Y} \subset D_{\overline{T^{*} \mathcal{W}_{Y}}}$. Thus this relative $K$-class is really an element of $K_{c}^{0}\left(T^{*} \mathcal{V}_{Y}\right)$. When $Q \in \mathcal{F}_{\text {Id }}^{-\infty}\left(\mathcal{W}_{Y} ; \underline{\mathbb{C}}^{n}\right)$, it is not hard to see that this construction reduces to definition 6.4 .

Coming back to $P$, this means that $P$ defines the same element in $K_{c}^{0}\left(T^{*} \mathcal{V}_{Y}\right)$ as $c(\operatorname{Id}+S)$. Now, since $P$ acts as a matrix near the boundary, it defines a vector bundle $\mathcal{E} \rightarrow \mathcal{V}_{Y}$ via the clutching construction and $P$ can be extended to act as the identity on $\left.\mathcal{E}\right|_{\mathcal{W}_{Y}^{\prime}}$. This defines a pseudodifferential operator $\widetilde{P} \in \Psi^{0}\left(\mathcal{V}_{Y} ; \mathcal{E}\right)$ having the same index as $P$. Moreover, it is not hard to see that the $K$-class in $K_{c}^{0}\left(T^{*} \mathcal{V}_{Y}\right)$ defined by the symbol of $\widetilde{P}$ is simply $c(\operatorname{Id}+S)$. By the Atiyah-Singer index theorem, the index of $\widetilde{P}$ is then given by $\operatorname{ind}_{t}(c(\operatorname{Id}+S))$, which completes the proof.

The formula for the index is now easy to get.

Theorem 6.6. The index of a Fredholm operator $(\operatorname{Id}+A) \in \mathcal{F}_{\Phi}^{-\infty}(X ; E)$ is given by

$$
\operatorname{ind}(\operatorname{Id}+A)=\operatorname{ind}_{t}(\kappa(\operatorname{Id}+A))
$$

where $\kappa(\operatorname{Id}+A) \in K_{c}^{0}\left(T^{*} Y\right)$ is the associated $K$-class of definition 5.23 and

$$
\operatorname{ind}_{t}: K_{c}^{0}\left(T^{*} Y\right) \rightarrow \mathbb{Z}
$$

is the topological index map of Atiyah and Singer.

Proof. By lemma 6.3, for $n \in \mathbb{N}$ large enough, there exists a scattering operator $(\operatorname{Id}+S) \in \mathcal{F}_{\mathrm{Id}}^{-\infty}\left(\mathcal{W}_{Y} ; \underline{\mathbb{C}}^{n}\right)$ which has the same index as $\mathrm{Id}+A$ and such that $\kappa(\operatorname{Id}+S)=i_{1} \kappa(\operatorname{Id}+A)$. By the previous proposition, the index of $\operatorname{Id}+S$ is given by

$$
\operatorname{ind}(\operatorname{Id}+S)=\operatorname{ind}_{t}(c(\operatorname{Id}+S)) \text {, }
$$

where $c(\operatorname{Id}+S) \in K_{c}^{0}\left(T^{*} \mathcal{V}_{Y}\right)$ is defined using the clutching construction. At $Y_{0} \subset$ $\partial \mathcal{W}_{Y}$, the indicial family of $(\operatorname{Id}+S)$ is trivial. This means the clutching construction 
only depends on the part of the indicial family of $\operatorname{Id}+S$ defined over ${ }^{\operatorname{Id}} N^{*} Y_{1}$. Let

$$
c:\left[{ }^{\Phi} N^{*} Y ; \operatorname{GL}(n ; \mathbb{C})\right] \rightarrow K_{c}^{0}\left(T^{*} \mathcal{V}_{Y}\right)
$$

denote the map obtained by applying the clutching construction, where $Y$ is identified with $Y_{1} \subset \mathcal{V}_{Y}$. The identification

$$
\left[{ }^{\Phi} N^{*} Y ; \mathrm{GL}(\infty ; \mathbb{C})\right] \cong\left[\mathbb{R} \times T^{*} Y ; \mathrm{GL}(\infty ; \mathbb{C})\right] \cong \widetilde{K}^{-2}\left(Y^{T^{*} Y}\right)
$$

combined with (6.4) gives a map

$$
f: \widetilde{K}^{-2}\left(Y^{T^{*} Y}\right) \rightarrow K_{c}^{0}\left(T^{*} \mathcal{V}_{Y}\right)
$$

Let us decompose the map $f$ into simpler maps. Since $N\left(\left.T^{*} \mathcal{V}_{Y}\right|_{Y}\right) \cong \mathbb{R}^{2} \times T^{*} Y$, we see that there is an isomorphism

$$
j: \widetilde{K}^{-2}\left(Y^{T^{*} Y}\right) \stackrel{\sim}{\longrightarrow} K_{c}^{0}\left(N\left(\left.T^{*} \mathcal{V}_{Y}\right|_{Y}\right)\right) .
$$

On the other hand, a tubular neighborhood of $T^{*} Y_{1}$ in $T^{*} \mathcal{V}_{Y}$ defines an inclusion

$$
i: N\left(\left.T^{*} \mathcal{V}_{Y}\right|_{Y}\right) \hookrightarrow T^{*} \mathcal{V}_{Y}
$$

which in turn defines a push-forward map in $K$-theory

$$
i_{*}: K_{c}^{0}\left(N\left(\left.T^{*} \mathcal{V}_{Y}\right|_{Y}\right)\right) \rightarrow K_{c}^{0}\left(T^{*} \mathcal{V}_{Y}\right)
$$

Then, it is not hard to see that the map $f$ is given by

$$
f=i_{*} \circ j: \widetilde{K}^{-2}\left(Y^{T^{*} Y}\right) \rightarrow K_{c}^{0}\left(T^{*} \mathcal{V}_{Y}\right)
$$

Combined with the periodicity isomorphism $p: K_{c}^{0}\left(T^{*} Y\right) \stackrel{\sim}{\rightarrow} \widetilde{K}^{-2}\left(Y^{T^{*} Y}\right)$, we see that

$$
c(\operatorname{Id}+S)=i_{*} \circ j \circ p(\kappa(\operatorname{Id}+A)) .
$$

Now, the composition of maps $j \circ p$ is just the Thom isomorphism

$$
\varphi: K_{c}^{0}\left(T^{*} Y\right) \rightarrow K_{c}^{0}\left(N\left(\left.T^{*} \mathcal{V}_{Y}\right|_{Y}\right)\right)
$$

applied to the trivial bundle $\underline{\mathbb{C}}$ over $T^{*} Y$, since $N\left(\left.T^{*} \mathcal{V}_{Y}\right|_{Y}\right) \cong \mathbb{R}^{2} \times T^{*} Y$. Thus, one has

$$
c(\operatorname{Id}+S)=i_{!} \kappa(\operatorname{Id}+A)
$$


where $i_{!}=i_{*} \varphi$. From the commutativity of the diagram ${ }^{1}$

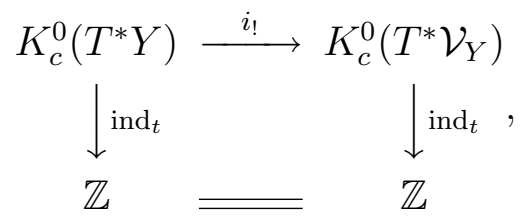

we conclude that

$$
\begin{aligned}
\operatorname{ind}(\operatorname{Id}+A) & =\operatorname{ind}(\operatorname{Id}+S) \\
& =\operatorname{ind}_{t} c(\operatorname{Id}+S)=\operatorname{ind}_{t} i_{!} \kappa(\operatorname{Id}+A) \\
& =\operatorname{ind}_{t} \kappa(\operatorname{Id}+A) .
\end{aligned}
$$

Corollary 6.7. When $\operatorname{dim} Z>0$, the index map ind $: \mathcal{F}_{\Phi}^{-\infty}(X ; E) \rightarrow \mathbb{Z}$ is surjective.

Proof. From theorem 6.6, it suffices to prove that the map

$$
\kappa: \mathcal{F}_{\Phi}^{-\infty}(X ; E) \rightarrow K_{c}^{0}\left(T^{*} Y\right)
$$

of definition 5.23 is surjective, which is equivalent to showing that any homotopy class in $\left[{ }^{\Phi} N^{*} Y ; \mathrm{GL}(\infty, \mathbb{C})\right]$ can be realized through definition 5.10 as $[\mathrm{Id}+A]_{\infty}$ for some $(\operatorname{Id}+A) \in \mathcal{F}_{\Phi}^{-\infty}(X ; E)$.

So let $[f] \in\left[{ }^{\Phi} N^{*} Y ; \operatorname{GL}(\infty, \mathbb{C})\right]$ be arbritrary. Then, there exists $n \in \mathbb{N}$ depending on $[f]$ so that the homotopy class $[f]$ has a smooth representative

$$
f:{ }^{\Phi} N^{*} Y \rightarrow \mathrm{GL}(n, \mathbb{C}) \subset \mathrm{GL}(\infty, \mathbb{C})
$$

Considering some family of Laplacians as in the beginning of section 5 and using proposition 5.3, there exists an associated spectral section $\left(\Pi, R_{1}, R_{2}\right)$ with range a trivial complex vector bundle $\underline{\mathbb{C}}^{m} \rightarrow{ }^{\Phi} N^{*} Y$ with $m \geq n$. By taking $n$ larger if needed, we can assume $m=n$. Thus taking $(\operatorname{Id}+A) \in \mathcal{F}_{\Phi}^{-\infty}(X ; E)$ so that

$$
\begin{gathered}
\Pi_{b}\left(\mathrm{Id}+\widehat{A}_{0}(b)\right) \Pi_{b}=f(b), \quad \forall b \in{ }^{\Phi} N^{*} Y, \\
\left(\mathrm{Id}-\Pi_{b}\right)\left(\widehat{A}_{0}(b)\right)\left(\mathrm{Id}-\Pi_{b}\right)=0, \quad \forall b \in{ }^{\Phi} N^{*} Y,
\end{gathered}
$$

we see that $[\operatorname{Id}+A]_{\infty}=[f]$.

Corollary 6.8. When $\operatorname{dim} Z=0$ the index map ind $: \mathcal{F}_{\Phi}^{-\infty}\left(X ; \underline{\mathbb{C}}^{n}\right) \rightarrow \mathbb{Z}$ is surjective provided $n \in \mathbb{N}$ is large enough.

Proof. As in the previous corollary, it suffices to show that any homotopy class in $\left[{ }^{\Phi} N^{*} Y ; \mathrm{GL}(\infty, \mathbb{C})\right]$ can be written as $[\mathrm{Id}+A]$ for some $\operatorname{Id}+A \in \mathcal{F}_{\Phi}^{-\infty}\left(X ; \underline{\mathbb{C}}^{n}\right)$. But there exists $n \in \mathbb{N}$ depending on $Y$ so that any homotopy class in $\left[{ }^{\Phi} N^{*} Y ; \operatorname{GL}(\infty, \mathbb{C})\right]$

\footnotetext{
${ }^{1}$ see p.501 in $[2]$
} 
can be represented by a map from ${ }^{\Phi} N^{*} Y$ to $\operatorname{GL}(n, \mathbb{C})$, and clearly such maps can always be chosen to be the indicial family of some Fredholm operator in $\mathcal{F}_{\Phi}^{-\infty}\left(X ; \underline{\mathbb{C}}^{n}\right)$.

\subsection{The Index in the case $Y=\mathbb{S}^{1}$}

In the particular case where $Y=\mathbb{S}^{1}$, it is possible to obtain an index formula by direct computation. Indeed, in that case, the $*$-product of proposition 4.12 simplifies substantially. Moreover, by proposition 4.8 and the use of spectral sections as in chapter 5 , one can always reduce to the case of a trivial fibration. In what follows, let $\theta$ denote the angular variable on

$$
\mathbb{S}^{1}=\left\{e^{i \theta} \quad \mid \quad \theta \in \mathbb{R}\right\} \subset \mathbb{C}
$$

and let $\eta$ denote the cotangent variable on $T^{*} \mathbb{S}^{1}$.

Proposition 6.9. In the case of a trivial fibration with $Y=\mathbb{S}^{1}$, the $*$-product of proposition 4.12 is given by

$$
\begin{aligned}
a * b= & a b+x\left(i \partial_{\eta} a \partial_{\theta} b+i \eta \partial_{\tau} a \partial_{\eta} b+2 i \tau \partial_{\tau} a \partial_{\tau} b\right)+x^{2}\left(\partial_{\eta}^{2} a \partial_{\theta}^{2} b+i \partial_{\tau} a \partial_{x} b\right)+\mathcal{O}\left(x^{3}\right) \\
= & a_{0} b_{0}+x\left(i \partial_{\eta} a_{0} \partial_{\theta} b_{0}+i \eta \partial_{\tau} a_{0} \partial_{\eta} b_{0}+a_{0} b_{1}+a_{1} b_{0}+2 i \tau \partial_{\tau} a_{0} \partial_{\tau} b_{0}\right) \\
& +x^{2}\left(\partial_{\eta}^{2} a_{0} \partial_{\theta}^{2} b_{0}+i \partial_{\tau} a_{0} b_{1}+a_{0} b_{2}+a_{2} b_{0}+a_{1} b_{1}+i \partial_{\eta} a_{1} \partial_{\theta} b_{0}+i \eta \partial_{\tau} a_{1} \partial_{\eta} b_{0}\right. \\
& \left.\quad+i \partial_{\eta} a_{0} \partial_{\theta} b_{1}+i \eta \partial_{\tau} a_{0} \partial_{\eta} b_{1}+2 i \tau \partial_{\tau} a_{1} \partial_{\tau} b_{0}+2 i \tau \partial_{\tau} a_{0} \partial_{\tau} b_{1}\right)+\mathcal{O}\left(x^{3}\right) .
\end{aligned}
$$

For a proof of this result, we refer to lemma 10 in [9]. Notice however that in order to be consistent with our definition of the indicial family in (3.11), we had to adopt a different sign convention than the one chosen in [9].

Theorem 6.10. Given $P \in \mathcal{F}_{\Phi}^{-\infty}(X ; E)$, let $p: T^{*} \mathbb{S}^{1} \times \mathbb{R} \rightarrow \mathrm{GL}(\infty, \mathbb{C})$ be a smooth representative of the associated $K$-class $\kappa(P) \in K_{c}^{0}\left(T^{*} Y\right)$ seen as a homotopy class in $\left[T^{*} \mathbb{S}^{1} \times \mathbb{R} ; \mathrm{GL}(\infty, \mathbb{C})\right]$. Then the index of $P$ is given by

$$
\operatorname{ind}(P)=\frac{1}{6(2 \pi i)^{2}} \int_{T^{*} \mathbb{S}^{1} \times \mathbb{R}} \operatorname{Tr}\left[\left(p^{-1} d p\right)^{3}\right] .
$$

Proof. Deform $P$ so that $\widehat{N_{\Phi}^{\prime}(P)}=p$. Let $B \in \mathcal{F}_{\Phi}^{-\infty}(X ; E)$ be a parametrix of $P$ and denote by $b=\widehat{N_{\Phi}^{\prime}(B)}$ the full indicial family of $B$. From proposition 4.7 we need to compute $\left(N_{\Phi}^{\prime}\left(\widehat{D_{\log x} P}\right) B\right)_{2}$. The most straighforward way is probably by using the $*$-product. From $p * b=\mathrm{Id}$, we deduce that

$$
b_{0}=p^{-1}, \quad b_{1}=-i p^{-1}\left(\partial_{\eta} p\right)\left(\partial_{\theta} p^{-1}\right)-i \eta p^{-1}\left(\partial_{\tau} p\right)\left(\partial_{\eta} p^{-1}\right) .
$$


By lemma 4.13,

$$
N_{\Phi}^{\prime} \widehat{\left(D_{\log x} P\right)}=\sum_{k=1}^{\infty} \frac{x^{k} D_{\tau}^{k} p}{k}
$$

so

$$
\begin{aligned}
\left(N_{\Phi}^{\prime}\left(\widehat{D_{\log x} P}\right) B\right)_{2}= & \frac{1}{2}\left(D_{\tau}^{2} p\right) b_{0}+\left(D_{\tau} p\right) b_{1}+i\left(\partial_{\eta} D_{\tau} p\right)\left(\partial_{\theta} b_{0}\right)+i \eta\left(\partial_{\tau} D_{\tau} p\right)\left(\partial_{\eta} b_{0}\right) \\
& +2 i \tau\left(\partial_{t a u} D_{\tau} p\right)\left(\partial_{\tau} b_{0}\right) \\
= & -\frac{1}{2}\left(\partial_{\tau}^{2} p\right) b_{0}-\left(\partial_{\tau} p\right)\left(p^{-1}\left(\partial_{\eta} p\right)\left(\partial_{\theta} p^{-1}\right)+\eta p^{-1}\left(\partial_{\tau} p\right)\left(\partial_{\eta} p^{-1}\right)\right) \\
& +\left(\partial_{\eta} \partial_{\tau} p\right)\left(\partial_{\theta} p^{-1}\right)+\eta\left(\partial_{\tau}^{2} p\right)\left(\partial_{\eta} p^{-1}\right)+2 \tau\left(\partial_{\tau}^{2} p\right)\left(\partial_{\tau} p^{-1}\right) .
\end{aligned}
$$

Hence, according to proposition 4.7,

$$
\begin{aligned}
\operatorname{ind}(P) & =\frac{1}{(2 \pi)^{2}} \int_{T^{*} \mathbb{S}^{1} \times \mathbb{R}} \operatorname{Tr}\left[-\frac{1}{2}\left(\partial_{\tau}^{2} p\right) b_{0}+\left(\partial_{\eta} \partial_{\tau} p\right)\left(\partial_{\theta} p^{-1}\right)+2 \tau\left(\partial_{\tau}^{2} p\right)\left(\partial_{\tau} p^{-1}\right)\right. \\
- & \left.\left(\partial_{\tau} p\right)\left(p^{-1}\left(\partial_{\eta} p\right)\left(\partial_{\theta} p^{-1}\right)+\eta p^{-1}\left(\partial_{\tau} p\right)\left(\partial_{\eta} p^{-1}\right)\right)+\eta\left(\partial_{\tau}^{2} p\right)\left(\partial_{\eta} p^{-1}\right)\right] d \theta d \eta d \tau .
\end{aligned}
$$

This is a complicated expression, but most of the terms do not contribute. Indeed, since the index only depends on the homotopy class of the indicial family $p$, one can consider a rescaled indicial family in the $\tau$ variable

$$
p_{\lambda}(\theta, \eta, \tau)=p(\theta, \eta, \lambda \tau), \quad \lambda \in \mathbb{R}^{+} .
$$

Since for all $\lambda \in \mathbb{R}^{+}$we should obtain the same index by using $p_{\lambda}$, this gives

$$
\begin{aligned}
0=\frac{(\lambda-1)}{(2 \pi)^{2}} \int_{T^{*} \mathbb{S}^{1} \times \mathbb{R}} \operatorname{Tr}[ & -\frac{1}{2}\left(\partial_{\tau}^{2} p\right) b_{0}+\eta\left(\partial_{\tau}^{2} p\right)\left(\partial_{\eta} p^{-1}\right) \\
& \left.-\left(\partial_{\tau} p\right) \eta p^{-1}\left(\partial_{\tau} p\right)\left(\partial_{\eta} p^{-1}\right)+2 \tau\left(\partial_{\tau}^{2} p\right)\left(\partial_{\tau} p^{-1}\right)\right] d \theta d \eta d \tau .
\end{aligned}
$$

Hence, the index is given by

$$
\operatorname{ind}(P)=\frac{1}{(2 \pi)^{2}} \int_{T^{*} \mathbb{S}^{1} \times \mathbb{R}} \operatorname{Tr}\left[\left(\partial_{\eta} \partial_{\tau} p\right)\left(\partial_{\theta} p^{-1}\right)-\left(\partial_{\tau} p\right) p^{-1}\left(\partial_{\eta} p\right)\left(\partial_{\theta} p^{-1}\right)\right] d \theta d \eta d \tau \text {. }
$$

Integrating by part with respect to $\eta$ in the first term, this becomes

$$
\begin{aligned}
& \operatorname{ind}(P)= \\
& \frac{1}{(2 \pi)^{2}} \int_{T^{*} \mathbb{S}^{1} \times \mathbb{R}} \operatorname{Tr}\left[-\left(p^{-1} \partial_{\eta} p\right)\left(p^{-1} \partial_{\tau} p\right)\left(p^{-1} \partial_{\theta} p\right)+\left(p^{-1} \partial_{\tau} p\right)\left(p^{-1} \partial_{\eta} \partial_{\theta} p\right)\right] d \theta d \eta d \tau .
\end{aligned}
$$


On the other hand, we can use another formula to compute the index,

$$
\begin{aligned}
\operatorname{ind}(P) & =\overline{\operatorname{Tr}}([P, B])=-\overline{\operatorname{Tr}}([B, P])=-\operatorname{Tr}_{\mathrm{R}}\left(D_{\log x} B P\right) \\
& =-\frac{1}{(2 \pi)^{2}} \int_{T^{*} \mathbb{S}^{1} \times \mathbb{R}} \operatorname{Tr}\left[\left(\widehat{D_{\log x} B}\right)_{2}\right] d \theta d \eta d \tau
\end{aligned}
$$

But using the fact that

$$
N_{\Phi}^{\prime} \widehat{\left(D_{\log x} B\right)}=\sum_{k=1}^{\infty} \frac{x^{k}}{k} D_{\tau}^{k} b=\sum_{k=1}^{\infty} \frac{x^{k}}{k} D_{\tau}^{k} b_{0}+\sum_{k=1}^{\infty} \frac{x^{k+1}}{k} D_{\tau}^{k} b_{1}+\mathcal{O}\left(x^{3}\right),
$$

one can obtain

$$
\begin{aligned}
\left(\widehat{D_{\log x} B} P\right)_{2}=\left(\frac{1}{2} D_{\tau}^{2} b_{0}+D_{\tau} b_{1}\right) p+ & i\left(\partial_{\eta} D_{\tau} b_{0}\right) \partial_{\theta} p \\
& +i \eta\left(\partial_{\tau} D_{\tau} b_{0}\right) \partial_{\eta} p+2 i \tau\left(\partial_{\tau} D_{\tau} b_{0}\right)\left(\partial_{\tau} p\right) .
\end{aligned}
$$

Using the same kind of rescaling argument in $\tau$, many terms do not contribute and one gets

$$
\operatorname{ind}(P)=-\frac{1}{(2 \pi)^{2}} \int_{T^{*} \mathbb{S}^{1} \times \mathbb{R}} \operatorname{Tr}\left[-\partial_{\tau}\left(p^{-1} \partial_{\eta} p \partial_{\theta} p^{-1}\right) p+\left(\partial_{\eta} \partial_{\tau} p^{-1}\right) \partial_{\theta} p\right] d \theta d \eta d \tau .
$$

Integrating by part with respect to $\tau$ in the first term and with respect to $\eta$ in the second term, one gets

$$
\begin{aligned}
& \operatorname{ind}(P)= \\
& \quad-\frac{1}{(2 \pi)^{2}} \int_{T^{*} \mathbb{S}^{1} \times \mathbb{R}} \operatorname{Tr}\left[-\left(p^{-1} \partial_{\eta} p\right)\left(p^{-1} \partial_{\theta} p\right)\left(p^{-1} \partial_{\tau} p\right)+\left(p^{-1} \partial_{\tau} p\right)\left(p^{-1} \partial_{\eta} \partial_{\theta} p\right)\right] d \theta d \eta d \tau .
\end{aligned}
$$

Adding together (6.7) and (6.9), we finally get

$$
\begin{aligned}
\operatorname{ind}(P)= & \frac{1}{2(2 \pi)^{2}} \int_{T^{*} \mathbb{S}^{1} \times \mathbb{R}} \operatorname{Tr}\left[\left(p^{-1} \partial_{\eta} p\right)\left(p^{-1} \partial_{\theta} p\right)\left(p^{-1} \partial_{\tau} p\right)\right. \\
& \left.-\left(p^{-1} \partial_{\eta} p\right)\left(p^{-1} \partial_{\tau} p\right)\left(p^{-1} \partial_{\theta} p\right)\right] d \theta d \eta d \tau \\
= & -\frac{1}{(2 \pi)^{2}} \int_{T^{*} \mathbb{S}^{1} \times \mathbb{R}} \operatorname{Tr}\left[\left(p^{-1} d p\right)^{3}\right] \\
= & \frac{1}{(2 \pi i)^{2}} \int_{T^{*} \mathbb{S}^{1} \times \mathbb{R}} \operatorname{Tr}\left[\left(p^{-1} d p\right)^{3}\right] .
\end{aligned}
$$

Remark 6.11. This formula is in agreement with theorem 6.6. Indeed, notice that the one point compactification of $T^{*} \mathbb{S}^{1} \times \mathbb{R}$ is homeomorphic to $\mathbb{S}^{3} / \mathbb{S}^{1}$, so the formula 
can be rewritten as

$$
\operatorname{ind}(P)=\int_{\mathbb{S}^{3} / \mathbb{S}^{1}} p^{*}\left(\beta_{2}^{\text {odd }}\right) .
$$

This is the isomorphism $\pi_{3}(\mathrm{GL}(\infty, \mathbb{C})) \cong \mathbb{Z}$ of proposition 7.24 and this corresponds to the index map. 


\section{Chapter 7}

\section{The Homotopy Groups of $G_{\Phi}^{-\infty}$}

. When homotopy groups come into play, the cases where $\operatorname{dim} Z>0$ and where $\operatorname{dim} Z=0$ are radically different. In some sense, the case where $\operatorname{dim} Z>0$ is to the case where $\operatorname{dim} Z=0$ what stable homotopy groups of $\operatorname{GL}(n, \mathbb{C})$ are to the actual homotopy groups of $\mathrm{GL}(n, \mathbb{C})$. That is to say, the homotopy groups are much easier to describe in the first case. What explains this difference is that the bundle $\pi^{*} G^{-\infty}$ is infinite dimensional in the case where $\operatorname{dim} Z>0$. This gives more freedom in the way one can perform homotopies, which has the effect of reducing the set of homotopy classes, and thus of simplifying the picture.

Still, it is possbible to say something in the case where $\operatorname{dim} Z=0$ if one consents to allow some stabilization. This goes as follows. Let $X$ be a compact manifold with boundary $\partial X$ together with a fibred cusp structure given by a finite covering $\Phi: \partial X \rightarrow Y$. Then consider the manifold with boundary $\mathbb{S}^{1} \times X$ with fibred cusp structure given by

$$
\begin{aligned}
\Phi_{\mathbb{S}^{1}}: \mathbb{S}^{1} \times X & \rightarrow Y \\
(s, p) & \mapsto \Phi(p) .
\end{aligned}
$$

Let $E \rightarrow X$ be any complex vector bundle over $X$. Then $E$ can be seen as a subbundle of the (trivial) Hilbert bundle $\mathrm{L}^{2}\left(S^{1}\right)$ over $X$, and Fredholm operators in $\mathcal{F}_{\Phi}^{-\infty}(X ; E)$ can be extended by letting them act as the identity on the complement of $E$ in $\mathrm{L}^{2}\left(\mathbb{S}^{1}\right) \rightarrow X$. This gives an embedding

$$
\mathcal{F}_{\Phi}^{-\infty}(X ; E) \subset \mathcal{F}_{\Phi_{\mathbb{S}}}^{-\infty}\left(\mathbb{S}^{1} \times X ; \underline{\mathbb{C}}\right) .
$$

In this way, the space $\mathcal{F}_{\Phi_{\mathbb{S} 1}}^{-\infty}\left(\mathbb{S}^{1} \times X ; \underline{\mathbb{C}}\right)$ is a stabilization of $\mathcal{F}_{\Phi}^{-\infty}(X ; E)$ which brings us back to the case where $\operatorname{dim} Z>0$. To the reader not completely satisfied by this compromise, let us point out where our $\operatorname{argument}$ breaks down when $\operatorname{dim} Z=0$. It is in the failure of being able to prove lemma 7.17, which is an important ingredient in the proof of the surjectivity of the boundary homomorphism occurring in the long exact sequence $(7.2)$. 


\subsection{Various spaces and a related Serre fibration}

From now on, we will assume that $\operatorname{dim} Z>0$.

Definition 7.1. Let $G_{\Phi}^{-\infty}(X ; E) \subset \mathcal{F}_{\Phi}^{-\infty}(X ; E)$ be the group of invertible operators in $\mathcal{F}_{\Phi}^{-\infty}(X ; E)$, that is,

$$
G_{\Phi}^{-\infty}(X ; E)=\left\{\operatorname{Id}+A \mid A \in \Psi_{\Phi}^{-\infty}(X ; E) \text { and }(\operatorname{Id}+A) \text { is invertible }\right\} .
$$

We want to compute the homotopy groups of $G_{\Phi}^{-\infty}(X ; E)$. Our approach will be similar to the one in [18] in the sense that we will compute the homotopy groups of $G_{\Phi}^{-\infty}(X ; E)$ out of a long exact sequence of homotopy groups. To this end, let us introduce the other spaces involved.

Definition 7.2. Let $\dot{G}^{-\infty}(X ; E)$ be the group of operators given by

$$
\dot{G}^{-\infty}(X ; E)=\left\{\operatorname{Id}+Q \mid Q \in x^{\infty} \Psi_{\Phi}^{-\infty}(X ; E), \operatorname{Id}+Q \text { is invertible }\right\} .
$$

Remark 7.3. By considering the eigensections vanishing in Taylor series at the boundary of a Laplacian arising from a fibred cusp metric (see (8.1) in [12]), one can identify $\dot{G}^{-\infty}(X ; E)$ with $\mathcal{G}^{-\infty}$, the group of semi-infinite invertible matrices introduced in definition 2.24. This means $\dot{G}^{-\infty}(X ; E)$ is a classifying space for odd $K$-theory. Notice also that $\dot{G}^{-\infty}(X ; E)$ does not depend on the choice of a fibred cusp structure.

Definition 7.4. Let $G_{\Phi s}^{-\infty}(\partial X ; E)$ denote the group

$$
\left\{\operatorname{Id}+Q \mid Q \in \Psi_{\Phi s}^{-\infty}(\partial X ; E) \text { and } \operatorname{Id}+Q \text { is invertible }\right\},
$$

that is, the group of invertible ${ }^{\Phi} N Y$-suspended smoothing perturbations of the identity. Taking the Fourier transform in ${ }^{\Phi} N Y$, this group naturally identitfies with the group $\Gamma_{\mathrm{Id}}\left({ }^{\Phi} N^{*} Y ; \pi^{*} G^{-\infty}\right)$ of smooth sections of $\pi^{*} G^{-\infty}$ asymptotic to the identity. This is the point of view we will adopt.

Lemma 7.5. The homotopy groups of $G_{\Phi s}^{-\infty}(\partial X ; E)$ are:

$$
\pi_{k}\left(G_{\Phi s}^{-\infty}(\partial X ; E)\right) \cong \begin{cases}K_{c}^{0}\left(T^{*} Y\right) & , \quad k \text { even } \\ \widetilde{K}^{-1}\left(Y^{T^{*} Y}\right) & , \text { k odd }\end{cases}
$$

Proof. By proposition 5.17, we have

$$
\begin{aligned}
\pi_{k}\left(G_{\Phi s}^{-\infty}(\partial X ; E)\right) & =\pi_{k}\left(\Gamma_{\mathrm{Id}}\left({ }^{\Phi} N^{*} Y ; \pi^{*} G^{-\infty}\right)\right) \\
& \cong\left[{ }^{\Phi} N^{*} Y \times \mathbb{R}^{k} ; \pi^{*} G^{-\infty}\right] \\
& \cong\left[T^{*} Y \times \mathbb{R}^{k+1} ; \mathrm{GL}(\infty, \mathbb{C})\right] \\
& \cong \widetilde{K}^{-1}\left(\mathbb{S}^{k+1} \wedge Y^{T^{*} Y}\right)=\widetilde{K}^{-k-2}\left(Y^{T^{*} Y}\right) .
\end{aligned}
$$


The result then follows from the periodicity theorem of $K$-theory.

Proposition 7.6. The homotopy groups of $\mathcal{F}_{\Phi}^{-\infty}(X ; E)$ are the same as those of $G_{\Phi s}^{-\infty}(\partial X ; E)$, namely

$$
\pi_{k}\left(\mathcal{F}_{\Phi}^{-\infty}(X ; E)\right) \cong \begin{cases}K_{c}^{0}\left(T^{*} Y\right) & , \text { k even } \\ \widetilde{K}^{-1}\left(Y^{T^{*} Y}\right) & , \text { k odd }\end{cases}
$$

Proof. Consider the short exact sequence

$$
\operatorname{Id}+x \Psi_{\Phi}^{-\infty}(X ; E) \hookrightarrow \mathcal{F}_{\Phi}^{-\infty}(X ; E) \stackrel{N_{\Phi}}{\longrightarrow} G_{\Phi s}^{-\infty}(\partial X ; E)
$$

This is a Serre fibration. One can see this using Seeley extension for manifolds with corners and thinking in terms of Schwartz kernels. The space $\operatorname{Id}+x \Psi_{\Phi}^{-\infty}(X ; E)$ is obviously contractible. So we deduce from the associated long exact sequence of homotopy groups that for $k \in \mathbb{N}$,

$$
\pi_{k}\left(\mathcal{F}_{\Phi}^{-\infty}(X ; E)\right) \cong \pi_{k}\left(G_{\Phi s}^{-\infty}(\partial X ; E)\right) .
$$

That $\pi_{0}\left(\mathcal{F}_{\Phi}^{-\infty}(X ; E)\right) \cong \pi_{0}\left(G_{\Phi s}^{-\infty}(\partial X ; E)\right)$ is clear from the contractibility of the space $\left(\mathrm{Id}+x \Psi_{\Phi}^{-\infty}(X ; E)\right)$.

Definition 7.7. Let $\mathcal{F}_{\Phi, 0}^{-\infty}(X ; E)$ denote the subspace of $\mathcal{F}_{\Phi}^{-\infty}(X ; E)$ consisting of operators having index zero. Let $G_{\Phi s, 0}^{-\infty}(\partial X ; E)$ be the subgroup of $G_{\Phi s}^{-\infty}(\partial X ; E)$ given by

$$
\left.G_{\Phi s, 0}^{-\infty}(\partial X ; E)=N_{\Phi}\left(\widehat{\mathcal{F}_{\Phi, 0}^{-\infty}(X} ; E\right)\right),
$$

and let $G_{\Phi s, 0}^{-\infty}(X ; E)[[x]]$ denotes the group of operators

$$
\left.G_{\Phi s, 0}^{-\infty}(X ; E)[[x]]=N_{\Phi}^{\prime}\left(\widehat{\mathcal{F}_{\Phi, 0}^{-\infty}(X} ; E\right)\right)
$$

where the composition law is given by the *-product of definition 4.11.

Remark 7.8. Notice that $G_{\Phi s, 0}^{-\infty}(\partial X ; E)$ has the same homotopy groups as the space $G_{\Phi s}^{-\infty}(\partial X ; E)$ but fewer connected components, more precisely,

$$
\pi_{0}\left(G_{\Phi s, 0}^{-\infty}(\partial X ; E)\right) \cong \pi_{0}\left(\mathcal{F}_{\Phi, 0}^{-\infty}(X ; E)\right) \cong \operatorname{ker}\left[\operatorname{ind}_{t}: K_{c}^{0}\left(T^{*} Y\right) \rightarrow \mathbb{Z}\right] .
$$

Moreover, keeping only the term of order $x^{0}$ gives a deformation retraction of the space $G_{\Phi s, 0}^{-\infty}(\partial X ; E)[[x]]$ onto $G_{\Phi s, 0}^{-\infty}(\partial X ; E)$, so $G_{\Phi s, 0}^{-\infty}(\partial X ; E)[[x]]$ as the same homotopy groups and the same set of connected components as $G_{\Phi s, 0}^{-\infty}(X ; E)$.

Lemma 7.9. The full normal operator $N_{\Phi}^{\prime}$ gives rise to a short exact sequence

$$
\dot{G}^{-\infty}(X ; E) \hookrightarrow G_{\Phi}^{-\infty}(X ; E) \stackrel{N_{\Phi}^{\prime}}{\longrightarrow} G_{\Phi s, 0}^{-\infty}(X ; E)[[x]]
$$

which is a Serre fibration. 
Proof. The injectivity and the exactness in the middle are clear. For the surjectivity, let $(\operatorname{Id}+Q) \in G_{\Phi s, 0}^{-\infty}(X ; E)[[x]]$ be arbitrary, and let $(\operatorname{Id}+P) \in \mathcal{F}_{\Phi, 0}^{-\infty}(X ; E)$ be such that $\widehat{N_{\Phi}^{\prime}(P)}=Q$. By proposition 3.15, the kernel and the cokernel of $\mathrm{Id}+P$ are contained in $\dot{\mathcal{C}}^{\infty}(X ; E)$. Since Id $+P$ has index zero, there exists a linear isomorphism $\varphi: \operatorname{ker} P \rightarrow$ coker $P$. By extending it to act as 0 on the orthogonal complement of ker $P$ in $\mathrm{L}^{2}(X ; E)$, it can be seen as an element of $x^{\infty} \Psi_{\Phi}^{-\infty}(X ; E)$, so that

$$
\mathrm{Id}+P+\varphi \in G_{\Phi}^{-\infty}(X ; E), \quad \widehat{N_{\Phi}^{\prime}} \widehat{(P+\varphi)}=Q .
$$

This shows that $N_{\Phi}^{\prime}$ is surjective. The proof that (7.1) is a Serre fibration is basically the same as the proof of lemma 3.5 in [18].

A Serre fibration has an associated long exact sequence of homotopy groups, so we have the following

Corollary 7.10. The Serre fibration (7.1) has an associated long exact sequence of homotopy groups

$$
\begin{array}{r}
\ldots \stackrel{\partial}{\longrightarrow} \pi_{k}\left(\dot{G}^{-\infty}\right) \longrightarrow \pi_{k}\left(G_{\Phi}^{-\infty}\right) \longrightarrow \pi_{k}\left(G_{\Phi s, 0}^{-\infty}\right) \stackrel{\partial}{\longrightarrow} \pi_{k-1}\left(\dot{G}^{-\infty}\right) \longrightarrow \cdots \\
\ldots \stackrel{\partial}{\longrightarrow} \pi_{0}\left(\dot{G}^{-\infty}\right) \longrightarrow \pi_{0}\left(G_{\Phi}^{-\infty}\right) \longrightarrow \pi_{0}\left(G_{\Phi s, 0}^{-\infty}\right),
\end{array}
$$

where $\partial$ is the boundary homomorphism and $\dot{G}^{-\infty}, G_{\Phi}^{-\infty}$ and $G_{\Phi s, 0}^{-\infty}$ denote respectively $\dot{G}^{-\infty}(X ; E), G_{\Phi}^{-\infty}(X ; E)$ and $G_{\Phi s, 0}^{-\infty}(\partial X ; E)[[x]]$.

Since we know the homotopy groups of $\dot{G}^{-\infty}(X ; E)$ and $G_{\Phi s, 0}^{-\infty}(X ; E)$, we will be able to compute the homotopy groups of $G_{\Phi}^{-\infty}(X ; E)$ provided we identify the boundary homomorphism $\partial$. In fact, it is only necessary to know that $\partial$ is surjective. But even with a complete knowledge of $\partial$, there would still be an ambiguity concerning the connected components of $G_{\Phi}^{-\infty}(X ; E)$. Let us get rid of this ambiguity immediately.

Lemma 7.11. The set of connected components of $G_{\Phi}^{-\infty}(X ; E)$ is given by

$$
\pi_{0}\left(G_{\Phi}^{-\infty}(X ; E)\right) \cong \operatorname{ker}\left[\operatorname{ind}_{t}: K_{c}^{0}\left(T^{*} Y\right) \rightarrow \mathbb{Z}\right]
$$

Proof. From proposition 7.6 and theorem 6.6, it suffices to prove that

$$
\pi_{0}\left(G_{\Phi}^{-\infty}(X ; E)\right) \longrightarrow \pi_{0}\left(\mathcal{F}_{\Phi}^{-\infty}(X ; E)\right) \stackrel{\text { ind }}{\longrightarrow} \mathbb{Z}
$$

is a short exact sequence. The surjectivity follows from corollary 6.7. The exactness in the middle follows using proposition 3.15 (cf. the proof of the surjectivity in lemma 7.9). Finally, the injectivity follows from the long exact sequence (7.2), the fact that $\pi_{0}\left(\dot{G}^{-\infty}(X ; E)\right)=\{0\}$ and proposition 7.6. 


\subsection{The boundary homomorphism seen as an in- dex map}

To prove that the boundary homomorphism is surjective, we will see it as a generalization of the index map ind : $\pi_{0}\left(\mathcal{F}_{\Phi}^{-\infty}(X ; E)\right) \rightarrow \mathbb{Z}$ to all homotopy groups of $\mathcal{F}_{\Phi}^{-\infty}(X ; E)$. Let us recall from the appendix in [1] how can one perform such a generalization.

Definition 7.12. If $M$ is a compact manifold with basepoint $m_{0}$, we denote by $\left[M ; \mathcal{F}_{\Phi}^{-\infty}(X ; E)\right]$ the set of homotopy classes of continuous maps from the manifold $M$ to the space $\mathcal{F}_{\Phi}^{-\infty}(X ; E)$ which send the basepoint $m_{0}$ to the identity.

We will define an index map

$$
\text { ind }:\left[M ; \mathcal{F}_{\Phi}^{-\infty}(X ; E)\right] \rightarrow \widetilde{K}^{0}(M)
$$

where $\widetilde{K}^{0}(M)$ is the reduced even $K$-theory of $M$. Let $H$ denote the Hilbert space $\mathrm{L}^{2}(X ; E)$ defined using some smooth positive density on $X$. Given a continuous map

$$
\begin{aligned}
T: M & \rightarrow \mathcal{F}_{\Phi}^{-\infty}(X ; E) \\
m & \mapsto T_{m},
\end{aligned}
$$

with $T_{m_{0}}=\mathrm{Id}$, one can show ${ }^{1}$ the existence of a vector space $V \subset H$ which is closed of finite codimension such that $V \cap \operatorname{ker} T_{m}=\{0\}$ for all $m \in M$, and such that

$$
\bigcup_{m \in M} H / T_{m}(V)
$$

topologized as a quotient space of $M \times H$, is a vector bundle over $M$. Let us denote this vector bundle by $H / T(V)$.

Definition 7.13. Let $T: M \rightarrow \mathcal{F}_{\Phi}^{-\infty}(X ; E)$ and $V \subset H$ be as above. Then the index of $T$ is defined to be

$$
\operatorname{ind}(T)=[H / V]-[H / T(V)] \in \widetilde{K}^{0}(M)
$$

One can check that the index of $T$ only depends on the homotopy class of $T$ and that it does not depend on the choice of $V \subset H$. Moreover, if $S: M \rightarrow \mathcal{F}_{\Phi}^{-\infty}(X ; E)$ is another continuous map, then

$$
\operatorname{ind}(T S)=\operatorname{ind}(T)+\operatorname{ind}(S) .
$$

For later convenience, we will give some precision about the way one can choose the vector space $V$ used in definition 7.13. First, notice that the orthogonal complement

\footnotetext{
${ }^{1}$ see proposition $A 5$ in [1]
} 
$V^{\perp}$ of $V$ in $H$ is naturally isomorphic to $H / V$. Moreover, the vector bundle $T(V)^{\perp} \rightarrow$ $M$ with fibre at $m \in M$ given by the orthogonal complement $T_{m}(V)^{\perp}$ of $T_{m}(V)$ is naturally isomorphic to the vector bundle $H / T(V)$. Hence, the index can also be written

$$
\operatorname{ind}(T)=\left[V^{\perp}\right]-\left[T(V)^{\perp}\right]
$$

Lemma 7.14. It is possible to choose the vector space $V$ occuring in definition 7.13 such that

$$
V^{\perp} \subset \dot{\mathcal{C}}^{\infty}(X ; E), T_{m}(V)^{\perp} \subset \dot{\mathcal{C}}^{\infty}(X ; E) \forall m \in M
$$

Moreover, if $\operatorname{ind}(T)=0$, we can also choose $V$ so that the vector bundles $V^{\perp}$ and $T(V)^{\perp}$ are isomorphic.

Proof. According to the proof of proposition $A 5$ in [1], one can always choose $V$ to be of the form

$$
V=\bigcap_{i=1}^{n}\left(\operatorname{ker} T_{m_{i}}\right)^{\perp} \Rightarrow V^{\perp}=\sum_{i=1}^{n} \operatorname{ker} T_{m_{i}},
$$

for some finite number of points $m_{1}, \ldots, m_{n} \in M$. Thus, by propositon $3.15, V^{\perp} \subset$ $\dot{\mathcal{C}}^{\infty}(X ; E)$. Moreover, for all $m \in M$,

$$
v \in T_{m}(V)^{\perp} \Rightarrow v \in \operatorname{ker} T_{m}^{*}+T_{m}\left(V^{\perp}\right)
$$

so by proposition 3.15 , this means $T_{m}(V)^{\perp} \subset \dot{\mathcal{C}}^{\infty}(X ; E)$.

If $\operatorname{ind}(T)=0$, then there exists a trivial bundle $P$ over $M$ so that

$$
H / V \oplus P \cong H / T(V) \oplus P .
$$

By taking a subspace $W \subset V$ such that $\operatorname{dim}(V / W)=\operatorname{rank}(P)$, we have

$$
H / W \cong H / T(W)
$$

Without loss of generality, we can assume as well that $W^{\perp} \subset \dot{\mathcal{C}}^{\infty}(X ; E)$, which implies as in (7.6) that $T_{m}(W)^{\perp} \subset \dot{\mathcal{C}}^{\infty}(X ; E)$ for all $m \in M$.

We know from proposition 7.6 that the normal operator $N_{\Phi}$ induces an isomorphism

$$
N_{\Phi *}: \pi_{k}\left(\mathcal{F}_{\Phi}^{-\infty}(X ; E)\right) \rightarrow \pi_{k}\left(G_{\Phi s}^{-\infty}(X ; E)\right)=\pi_{k}\left(G_{\Phi s, 0}^{-\infty}(X ; E)[[x]]\right), k \in \mathbb{N} .
$$

Moreover, since $\dot{G}^{-\infty}(X ; E)$ is a classifying space for odd $K$-theory, we get an isomorphism via the clutching construction

$$
c: \pi_{k}\left(\dot{G}^{-\infty}(X ; E)\right) \rightarrow \widetilde{K}^{-2}\left(\mathbb{S}^{k-1}\right)=\widetilde{K}^{0}\left(\mathbb{S}^{k+1}\right), k \in \mathbb{N} .
$$

Proposition 7.15. For $k \in \mathbb{N}$, the homomorphism

$$
c \circ \partial \circ N_{\Phi *}: \pi_{k}\left(\mathcal{F}_{\Phi}^{-\infty}(X ; E)\right) \rightarrow \widetilde{K}^{0}\left(\mathbb{S}^{k}\right)
$$


is the index map of definition 7.13 with $M=\mathbb{S}^{k}$.

Proof. Let $T: \mathbb{S}^{k} \rightarrow \mathcal{F}_{\Phi}^{-\infty}(X ; E)$ be a representative of an element of the homotopy group $\pi_{k}\left(\mathcal{F}_{\Phi}^{-\infty}(X ; E)\right)$. We assume that $T_{s_{0}}=\mathrm{Id}$, where $s_{0} \in \mathbb{S}^{k}$ is the basepoint of $\mathbb{S}^{k}$. By deforming $T$ if needed, we can assume that $T \equiv \mathrm{Id}$ in a open ball $B_{0}^{k} \subset \mathbb{S}^{k}$ containing $s_{0}$. Let the closed ball $\bar{B}_{1}^{k}$ be the complement of $B_{0}^{k}$ in $\mathbb{S}^{k}$.

Let $V \subset H$ be a closed subspace of $H$ of finite codimension that can be used to define the index of $\mathrm{T}$ :

$$
\operatorname{ind}(T)=[H / V]-[H / T(V)]=\left[V^{\perp}\right]-\left[T(V)^{\perp}\right] .
$$

By lemma 7.14, we can assume that $V^{\perp} \subset \dot{\mathcal{C}}^{\infty}(X ; E)$ and $T_{s}(V)^{\perp} \subset \dot{\mathcal{C}}^{\infty}(X ; E)$ for all $s \in \mathbb{S}^{k}$. When we restrict $T$ to the closed ball $\bar{B}_{1}^{k} \subset \mathbb{S}^{k}$, the index become zero because $\bar{B}_{1}^{k}$ is contractible and $T \equiv \mathrm{Id}$ on $\partial \bar{B}_{1}^{k}$. Thus, by lemma 7.14, we can also assume that $V$ is such that $V^{\perp}$ and $T(V)^{\perp}$ are isomorphic vector bundles when restricted to $\bar{B}_{1}^{k}$. Let $\varphi: V^{\perp} \rightarrow T(V)^{\perp}$ be a (smooth) isomorphism between $V^{\perp}$ and $T(V)^{\perp}$ on $\bar{B}_{1}^{k}$. By extending $\varphi$ to act by 0 on $V$, we get an associated family of bounded operator $\phi: \bar{B}_{1}^{k} \rightarrow \mathcal{L}(H, H)$. Since $V^{\perp} \subset \dot{\mathcal{C}}^{\infty}(X ; E)$ and $T_{s}(V)^{\perp} \subset \dot{\mathcal{C}}^{\infty}(X ; E)$ for all $s \in \mathbb{S}^{k}$, this is in fact a map

$$
\phi: \bar{B}_{1}^{k} \rightarrow x^{\infty} \Psi_{\Phi}^{-\infty}(X ; E) .
$$

By construction of $\phi$ and the compactness of $\bar{B}_{1}^{k}$, we see that for $\lambda>0$ large enough,

$$
T_{s}+\lambda \phi(s) \in G_{\Phi}^{-\infty}(X ; E), \forall s \in \bar{B}_{1}^{k} .
$$

Thus, rescaling $\phi$ if needed, we can assume $T+\phi$ is a map of the form

$$
T+\phi: \bar{B}_{1}^{k} \rightarrow G_{\Phi}^{-\infty}(X ; E) .
$$

Notice that $N_{\Phi}^{\prime} \circ(T+\phi)=N_{\Phi}^{\prime} \circ T$, so $T+\phi$ is in fact a lift of the map

$$
N_{\Phi}^{\prime} \circ T: \bar{B}_{1}^{k} \rightarrow G_{\Phi s, 0}^{-\infty}(X ; E)[[x]] .
$$

Moreover, since $T \equiv \operatorname{Id}$ on $\partial \bar{B}_{1}^{k} \cong \mathbb{S}^{k-1}$, the map $T+\phi$ takes value in $\dot{G}^{-\infty}(X ; E)$ when restricted to $\partial \bar{B}_{1}^{k}$. By definition of the boundary homomorphism (see for instance section 17.1 in [21]),

$$
\partial\left(\left[N_{\Phi}^{\prime} \circ T\right]\right)=\left[\left.(T+\phi)\right|_{\partial \bar{B}_{1}^{k}}\right] \in \pi_{k-1}\left(\dot{G}^{-\infty}(X ; E)\right) .
$$

Now, since $V^{\perp}=T(V)^{\perp}$ canonically on $\partial \bar{B}_{1}^{k}, \phi$ is just a map

$$
\phi: \partial \bar{B}_{1}^{k} \rightarrow \operatorname{End}\left(V^{\perp}, V^{\perp}\right)
$$

Clearly, the clutching construction applied to $(\mathrm{Id}+\phi)^{-1}$ gives the virtual bundle $\left[T(V)^{\perp}\right]-\left[V^{\perp}\right]$, which means that the clutching construction applied to $(\mathrm{Id}+\phi)$ gives 
$\left[V^{\perp}\right]-\left[T(V)^{\perp}\right]$. This shows that $c \partial N_{\Phi *}(T)=\operatorname{ind}(T)$, which concludes the proof.

\subsection{Surjectivity of the boundary homomorphism and the main theorem}

Since (7.7) and (7.8) are isomorphisms, the surjectivity of the boundary homomorphism is equivalent to the surjectivity of the index map of definition 7.13. In [1], there is a proof of the surjectivity of the index map, but considering the space $\mathcal{F}(H)$ of all Fredholm operators acting on $H$. It is possible to adapt this proof to our situation so that it still works. We first need to discuss further the topology of the space $\mathcal{F}_{\Phi}^{-\infty}(X ; E)$.

For $n \in \mathbb{N}$, consider the vector bundle $E^{n}=E \otimes \mathbb{C}^{n}$. The bundle $E$ can be seen as a subbundle of $E^{n}$ via the inclusion

$$
\begin{aligned}
i: E & \rightarrow E \otimes \mathbb{C}^{n} \\
e & \mapsto e \otimes(1, \underbrace{0, \ldots, 0}_{n-1 \text { times }}) .
\end{aligned}
$$

This gives an inclusion

$$
\begin{aligned}
i^{\prime}: \mathcal{F}_{\Phi}^{-\infty}(X ; E) & \hookrightarrow \mathcal{F}_{\Phi}^{-\infty}\left(X ; E^{n}\right) \\
\operatorname{Id}+A & \mapsto i^{\prime}(\operatorname{Id}+A),
\end{aligned}
$$

where $i^{\prime}(\operatorname{Id}+A)$ acts on sections of $E \subset E^{n}$ as $(\operatorname{Id}+A)$ does and acts on sections of of the complement of $E$ in $E^{n}$ as the identity.

Definition 7.16. A subspace $\mathcal{U}$ of a topological space $\mathcal{T}$ is said to be a weak deformation retract of $\mathcal{T}$ if for any closed manifold $M$ and any continuous map $f: M \rightarrow \mathcal{T}$, there exists a continuous map $g$ homotopic to $f$ such that $g(M) \subset \mathcal{U}$.

Lemma 7.17. Under the inclusion $(7.9), \mathcal{F}_{\Phi}^{-\infty}(X ; E)$ is a weak deformation retract of $\mathcal{F}_{\Phi}^{-\infty}\left(X ; E^{n}\right)$.

Proof. Let $M$ be a closed manifold and let $f: M \rightarrow \mathcal{F}_{\Phi}^{-\infty}\left(X ; E^{n}\right)$ be a continuous map. Without loss of generality, we can assume $f$ is smooth. Compose it with the normal operator $N_{\Phi}$ to get a map

$$
\tilde{f}=N_{\Phi} \circ f: M \rightarrow G_{\Phi s}^{-\infty}\left(\partial X ; E^{n}\right) .
$$

Let $\Delta_{\partial X / Y}^{E}$ be a family of Laplacians associated to the fibration $\Phi: \partial X \rightarrow Y$ and the complex vector bundle $E$ (not $E^{n}$ ). By the compactness of $M$, applying an argument similar to the proof of lemma 5.6, we see that there exists a spectral section $\left(\Pi, R_{1}, R_{2}\right)$ for $\Delta_{\partial X / Y}^{E}$ such that $\left(\Pi^{n}=\bigoplus_{i=1}^{n} \Pi, R_{1}, n R_{2}\right)$, which is a spectral section for $\bigoplus_{i=1}^{n} \Delta_{\partial X / Y}^{E}$, satisfies the estimate

$$
\left\|\widetilde{f}_{b}(m)-\operatorname{Id}-\Pi_{b}^{n}\left(\widetilde{f}_{b}(m)-\mathrm{Id}\right) \Pi_{b}^{n}\right\|<\frac{1}{2\left\|\widetilde{f}_{b}(m)^{-1}\right\|}, \quad \forall b \in{ }^{\Phi} N^{*} Y, m \in M .
$$


By the proof of corollary 5.7, this means that

$$
t \mapsto \tilde{f}_{t}=\tilde{f}+t\left(\Pi^{n}(\tilde{f}) \Pi^{n}-(\tilde{f}-\mathrm{Id})\right), \quad t \in[0,1],
$$

is a homotopy of smooth maps from $M$ to $G_{\Phi s}^{-\infty}(\partial X ; E)$.

Let $F$ be the range of $\Pi$. Taking $\Pi$ to have range with a larger rank if necessary, we can assume that $\widetilde{f}_{1}$ is homotopic, through smooth maps from $M$ to $\Gamma_{\text {Id }}\left({ }^{\Phi} N^{*} Y ; \pi^{*} \operatorname{GL}\left(F^{n}, \mathbb{C}\right)\right)$, to a map $\widetilde{f}_{2}$ with

$$
\widetilde{f}_{2}(M) \subset \Gamma_{\mathrm{Id}}\left({ }^{\Phi} N^{*} Y ; \pi^{*} \mathrm{GL}(F, \mathbb{C})\right),
$$

where $\mathrm{GL}(F, \mathbb{C}) \subset \mathrm{GL}\left(F^{n}, \mathbb{C}\right)$ acts as the identity on the complement of $F$ in $F^{n}$. In particular, this means that

$$
\widetilde{f}_{2}(M) \subset G_{\Phi s}^{-\infty}(\partial X ; E) \subset G_{\Phi s}^{-\infty}\left(\partial X ; E^{n}\right),
$$

where the inclusion $G_{\Phi s}^{-\infty}(\partial X ; E) \subset G_{\Phi s}^{-\infty}\left(\partial X ; E^{n}\right)$ is induced from the inclusion (7.9). Let $f_{2}: M \rightarrow \mathcal{F}_{\Phi}^{-\infty}(X ; E)$ be a map such that $\widehat{N_{\Phi}\left(f_{2}\right)}=\widetilde{f}_{2}$, which is possible by Seeley extension for manifold with corners. Then, using again Seeley extension, one can lift the homotopy between $\widetilde{f}=\widetilde{f}_{0}$ and $\widetilde{f}_{2}$ to a homotopy between $f$ and $f_{2}$ through smooth maps from $M$ to $\mathcal{F}_{\Phi}^{-\infty}\left(X ; E^{n}\right)$.

Proposition 7.18. For $k \in \mathbb{N}$, the boundary homomorphism

$$
\partial: \pi_{k}\left(G_{\Phi s, 0}^{-\infty}(X ; E)[[x]]\right) \rightarrow \pi_{k-1}\left(\dot{G}^{-\infty}(X ; E)\right)
$$

is surjective.

Proof. By proposition 7.15, it suffices to show that the index map

$$
\text { ind }: \pi_{k}\left(\mathcal{F}_{\Phi}^{-\infty}(X ; E)\right) \rightarrow \widetilde{K}^{0}\left(\mathbb{S}^{k}\right)
$$

is surjective.

Let $\mathcal{E} \rightarrow \mathbb{S}^{k}$ be an arbitrary complex vector bundle over $\mathbb{S}^{k}$. Let $\mathcal{F}$ be another complex vector bundle such that $\mathcal{E} \oplus \mathcal{F}=\underline{\mathbb{C}}^{n}$ is trivial. For $s \in \mathbb{S}^{k}$, let $p_{s} \in \operatorname{End}\left(\mathbb{C}^{n}\right)$ be the projection onto $\mathcal{E}_{s} \subset \mathbb{C}^{n}$.

Let $T_{-1} \in \mathcal{F}_{\Phi}^{-\infty}(X ; E)$ be an operator such that ind $\left(T_{-1}\right)=-1$. Such an operator exists by corollary 6.7. Consider the family of operators

$$
\widetilde{T}_{s}=T_{-1} \otimes p_{s}+\operatorname{Id} \otimes\left(1-p_{s}\right) \in \mathcal{F}_{\Phi}^{-\infty}\left(X ; E \otimes \underline{\mathbb{C}}^{n}\right), s \in \mathbb{S}^{k},
$$

acting on $H \otimes \mathbb{C}^{n}$. Clearly, ind $(\widetilde{T})=-[\mathcal{E}]$. By lemma 7.17 , we can deform the family $\widetilde{T}$ to a family

$$
R: \mathbb{S}^{k} \rightarrow \mathcal{F}_{\Phi}^{-\infty}(X ; E) \subset \mathcal{F}_{\Phi}^{-\infty}\left(X ; E \otimes \underline{\mathbb{C}}^{n}\right) .
$$

By homotopy invariance of the index, $\operatorname{ind}(R)=-[\mathcal{E}]$. If $\operatorname{rank}(\mathcal{E})=j$, let $T_{j} \in$ 
$\mathcal{F}_{\Phi}^{-\infty}(X ; E)$ be an operator of index $j$, then

$$
\operatorname{ind}\left(T_{j} \circ R\right)=\operatorname{ind}\left(T_{j}\right)+\operatorname{ind}(R)=[j]-[\mathcal{E}] .
$$

By construction, $T_{j} \circ R_{s}$ has index zero for all $s \in \mathbb{S}^{k}$. In particular, deforming $T_{j} \circ R$ if necessary, we can assume that $T_{j} \circ R_{s_{0}}$ is invertible at the basepoint $s_{0} \in \mathbb{S}^{k}$. Finally, consider the family

$$
\widetilde{R}=\left(T_{j} \circ R_{s_{0}}\right)^{-1}\left(T_{j} \circ R\right): \mathbb{S}^{k} \rightarrow \mathcal{F}_{\Phi}^{-\infty}(X ; E) .
$$

By construction, $\widetilde{R}_{s_{0}}=\mathrm{Id}$, and $\operatorname{ind}(\widetilde{R})=[j]-[\mathcal{E}]$. Since any element of $\widetilde{K}^{0}\left(\mathbb{S}^{k}\right)$ is of the form $[j]-[\mathcal{E}], j=\operatorname{rank} \mathcal{E}$, this shows that the index map is surjective.

Theorem 7.19. For $k \in \mathbb{N}_{0}$ and provided $\operatorname{dim} Z>0$,

$$
\begin{aligned}
& \pi_{2 k+1}\left(G_{\Phi}^{-\infty}(X ; E)\right) \cong \widetilde{K}^{-1}\left(Y^{T^{*} Y}\right) \\
& \pi_{2 k}\left(G_{\Phi}^{-\infty}(X ; E)\right) \cong \operatorname{ker}\left[\operatorname{ind}_{t}: K_{c}^{0}\left(T^{*} Y\right) \rightarrow \mathbb{Z}\right]
\end{aligned}
$$

Proof. The case of the set of connected components was handled in lemma 7.11. For the remaining cases, the surjectivity of the boundary homomorphism allows us to decompose the long exact sequence of homotopy groups (7.2) into short exact sequences. More precisely, for $k \in \mathbb{N}$, we get the exact sequences

$$
0 \longrightarrow \pi_{2 k-1}\left(G_{\Phi}^{-\infty}(X ; E)\right) \longrightarrow \pi_{2 k-1}\left(G_{\Phi s, 0}^{-\infty}(X ; E)\right) \longrightarrow 0
$$

and

$$
0 \longrightarrow \pi_{2 k}\left(G_{\Phi}^{-\infty}(X ; E)\right) \longrightarrow \pi_{2 k}\left(G_{\Phi s, 0}^{-\infty}(X ; E)\right) \stackrel{\partial}{\longrightarrow} \pi_{2 k-1}\left(\dot{G}^{-\infty}(X ; E)\right) \longrightarrow 0,
$$

where we used the fact that $\pi_{2 k}\left(\dot{G}^{-\infty}(X ; E)\right) \cong\{0\}$. For odd homotopy groups, the theorem easily follows from (7.10)and remark 7.8. For even homotopy groups, notice that by proposition 7.15 and proposition 7.6 , the exact sequence (7.11) can be rewritten

$$
0 \longrightarrow \pi_{2 k}\left(G_{\Phi}^{-\infty}(X ; E)\right) \longrightarrow \pi_{2 k}\left(\mathcal{F}_{\Phi}^{-\infty}(X ; E)\right) \stackrel{\text { ind }}{\longrightarrow} \widetilde{K}^{0}\left(\mathbb{S}^{2 k}\right) \longrightarrow 0,
$$

so $\pi_{2 k}\left(G_{\Phi}^{-\infty}(X ; E)\right) \cong \operatorname{ker}\left[\right.$ ind $\left.: \pi_{2 k}\left(\mathcal{F}_{\Phi}^{-\infty}(X ; E)\right) \rightarrow \widetilde{K}^{0}\left(\mathbb{S}^{k}\right)\right]$. But the homotopy group $\pi_{2 k}\left(\mathcal{F}_{\Phi}^{-\infty}(X ; E)\right)$ is isomorphic to $K_{c}^{0}\left(T^{*} Y\right)$, which is a finitely generated $\mathbb{Z}$ module, and $\widetilde{K}^{0}\left(\mathbb{S}^{k}\right) \cong \mathbb{Z}$. By the classification of finitely generated $\mathbb{Z}$-modules ${ }^{2}$, the isomorphism class of the kernel is the same for all surjective homomorphism $K_{c}^{0}\left(T^{*} Y\right) \rightarrow \mathbb{Z}$. Since $\operatorname{ind}_{t}: K_{c}^{0}\left(T^{*} Y\right) \rightarrow \mathbb{Z}$ is also surjective, this means

$$
\pi_{2 k}\left(G_{\Phi}^{-\infty}(X ; E)\right) \cong \operatorname{ker}\left[\operatorname{ind}_{t}: K_{c}^{0}\left(T^{*} Y\right) \rightarrow \mathbb{Z}\right]
$$

\footnotetext{
${ }^{2}$ See for instance in $[6]$.
} 


\subsection{A related exact sequence}

Let $\mathcal{H}$ be separable (infinite dimensional) Hilbert space, let $\mathcal{F}(\mathcal{H})$ denote the space of Fredholm operators acting from $\mathcal{H}$ to $\mathcal{H}$ and let $\mathcal{A}^{*}(\mathcal{H})$ denote the group of invertible bounded linear operators acting from $\mathcal{H}$ to $\mathcal{H}$. Then, given a compact space $M \in \mathcal{C}^{+}$ with basepoint $m_{0}$, there is an exact sequence

$$
\left[M ; \mathcal{A}^{*}(\mathcal{H})\right] \longrightarrow[M ; \mathcal{F}(\mathcal{H})] \stackrel{\text { ind }}{\longrightarrow} \widetilde{K}^{0}(M) \longrightarrow 0
$$

where ind is the index map of definition 7.13 (see proposition $A 6$ in [1]). In fact, by Kuiper's theorem, the group $\mathcal{A}^{*}(\mathcal{H})$ is contractible, so it is in fact a short exact sequence. The information of theorem 7.19 can be converted into a similar exact sequence by replacing $\mathcal{A}^{*}(\mathcal{H})$ by $G_{\Phi}^{-\infty}(X ; E)$ and $\mathcal{F}(\mathcal{H})$ by $\mathcal{F}_{\Phi}^{-\infty}(X ; E)$.

Corollary 7.20. Given a closed manifold $M$ with basepoint $m_{0}$, there is an exact sequence

$$
\left[M ; G_{\Phi}^{-\infty}(X ; E)\right] \longrightarrow\left[M ; \mathcal{F}_{\Phi}^{-\infty}(X ; E)\right] \stackrel{\text { ind }}{\longrightarrow} \widetilde{K}^{0}(M) \longrightarrow 0
$$

Proof. The surjectivity of the short exact sequence is given by the proof of proposition 7.18 with $\mathbb{S}^{k}$ replaced by $M$. For the exactness in the middle, it is clear that the index of a element of $\left[M ; G_{\Phi}^{-\infty}(X ; E)\right]$ is zero. On the other hand, assume that $T: M \rightarrow \mathcal{F}_{\Phi}^{-\infty}(X ; E)$ is such that ind $(T)=0$. We need to show that $T$ can be deformed to a map from $M$ to $G_{\Phi}^{-\infty}(X ; E)$. Let $V \subset \mathrm{L}^{2}(X ; E)$ be the vector space of lemma 7.14 and let $\varphi: V^{\perp} \rightarrow T\left(V^{\perp}\right)$ be an isomorphism. Since $T_{m_{0}}=\mathrm{Id}$, we can assume without loss of generality that $\varphi$ is the identity at the basepoint $m_{0}$. By extending $\varphi$ to act by zero on $V$, we get a map

$$
\phi: M \rightarrow x^{\infty} \Psi_{\Phi}^{-\infty}(X ; E)
$$

such that for $\lambda>0$ large enough,

$$
T_{m}+\lambda \phi(m) \in G_{\Phi}^{-\infty}(X ; E)
$$

for all $m \in M$ (cf. proof of proposition 7.15). Hence, by rescaling $\phi$ and deforming it near the basepoint $m_{0}$, we can assume that we have a map

$$
T+\phi: M \rightarrow G_{\Phi}^{-\infty}(X ; E)
$$

such that $T_{m_{0}}+\phi\left(m_{0}\right)=\mathrm{Id}$. Then

$$
t \mapsto T+t \phi, \quad t \in[0,1]
$$

is a homotopy between $T$ and $T+\phi$. This prove the exactness in the middle. 


\subsection{The particular case of cusp operators}

In the case of cusp operators, that is, when the fibration $\Phi: \partial X \rightarrow\{\mathrm{pt}\}$ is trivial, one gets as an easy corollary of theorem 7.19 the weak contractibility result of [18], namely that all the homotopy groups of $G_{\Phi}^{-\infty}(X ; E)$ vanish.

Corollary 7.21. If $\Phi: \partial X \rightarrow\{\mathrm{pt}\}$ is the trivial fibration (cusp operators), then $G_{\Phi}^{-\infty}(X ; E)$ is weakly contractible, meaning that all its homotopy groups are trivial, including the set of connected components.

Proof. In this case, $Y=\mathrm{pt}$, so $T^{*} Y$ does not really make sense, but looking back at the identification (5.19) and the proof of lemma 7.5, we see that $K_{c}^{0}\left(T^{*} Y\right)$ and $\widetilde{K}^{-1}\left(Y^{T^{*} Y}\right)$ must be replaced by $\widetilde{K}^{0}\left(\mathbb{S}^{2}\right) \cong \mathbb{Z}$ and $\widetilde{K}^{-1}\left(\mathbb{S}^{2}\right) \cong\{0\}$ in the statement of theorem 7.19. The result then easily follows from the fact that $\operatorname{ind}_{t}: \widetilde{K}^{0}\left(\mathbb{S}^{2}\right) \rightarrow \mathbb{Z}$ is an isomorphism.

In fact, it turns out that with only little more effort, one can deduce that the group $G_{\Phi}^{-\infty}(X ; E)$ is actually contractible. It suffices to apply with slight modifications the argument of Kuiper in his proof of the contractibility of the group of invertible bounded operators acting on a separable Hilbert space (see [8]).

The main difference in our situation is that the topology we consider on the group $G_{\Phi}^{-\infty}(X ; E)$ is not the one coming from the operator norm, but the $\mathcal{C}^{\infty}$-topology coming from the Schwartz kernels of the smoothing operators in $\Psi_{\Phi}^{-\infty}(X ; E)$. Nevertheless, since $G_{\Phi}^{-\infty}(X ; E) \subset \Psi_{\Phi}^{0}(X ; E)$, we can also give to $G_{\Phi}^{-\infty}(X ; E)$ the toplogy induced by the operator norm $\|\cdot\|$ of bounded operators acting on $\mathrm{L}^{2}(X ; E)$. This is a weaker topology then the $\mathcal{C}^{\infty}$-infinity topology, in the sense that it has less open sets. In the operator norm topology, the space $G_{\Phi}^{-\infty}(X ; E)$ is easily seen to be a metric space. By a theorem of Stone $^{3}$, metric spaces are paracompact. This will be very useful to retract $G_{\Phi}^{-\infty}(X ; E)$ (in the $\mathcal{C}^{\infty}$-topology) onto a $\mathrm{CW}$-complex.

Definition 7.22. We will say that an open ball in $\operatorname{Id}+\Psi_{\Phi}^{-\infty}(X ; E)$ of radius $\epsilon$

$$
B_{\epsilon}(A)=\left\{\operatorname{Id}+Q \mid Q \in \Psi_{\Phi}^{-\infty}(X ; E), \quad\|A-\operatorname{Id}-Q\|<\epsilon\right\}, \quad A \in G_{\Phi}^{-\infty}(X ; E),
$$

is small if $B_{3 \epsilon}(A) \subset G_{\Phi}^{-\infty}(X ; E)$. Clearly, one can cover $G_{\Phi}^{-\infty}(X ; E)$ by such balls.

Proposition 7.23. If $G_{\Phi}^{-\infty}(X ; E)$ is weakly contractible, then it is contractible. In particular, if $\Phi: \partial X \rightarrow \mathrm{pt}$ is the trivial fibration, then $G_{\Phi}^{-\infty}(X ; E)$ is contractible.

Proof. Let $\left\{B_{\epsilon_{i}}\left(A_{i}\right)\right\}_{i \in I}$ be a covering of $G_{\Phi}^{-\infty}(X ; E)$ by small balls,

$$
G_{\Phi}^{-\infty}(X ; E)=\bigcup_{i \in I} B_{\epsilon_{i}}\left(A_{i}\right)
$$

Since the Banach space of bounded operators acting on $\mathrm{L}^{2}(X ; E)$ is separable, we can assume $I=\mathbb{N}$. Moreover, since $G_{\Phi}^{-\infty}(X ; E)$ is paracompact in the operator norm

\footnotetext{
${ }^{3}$ See for instance theorem 4 , section $I .8 .4$, p.101 in [19]
} 
topology, we can assume that the covering (7.14) is locally finite and that it has an associated partition of unity $\left\{\phi_{i}\right\}_{i \in \mathbb{N}}$. A priori, the $\phi_{i}$ are continuous with respect to the operator norm topology, but since the $\mathcal{C}^{\infty}$-topology is a finer topology, this means they are also continuous with respect to the $\mathcal{C}^{\infty}$-topology. For $t \in[0,1]$, consider the following homotopy

$$
\begin{aligned}
\xi_{t}: \quad G_{\Phi}^{-\infty}(X ; E) & \rightarrow G_{\Phi}^{-\infty}(X ; E) \\
z & \mapsto(1-t) z+t \sum_{i \in \mathbb{N}} \phi_{i}(z) A_{i} .
\end{aligned}
$$

For $t=0, \xi_{0}$ is just the identity. To see that the image really lies in $G_{\Phi}^{-\infty}(X ; E)$, let $z \in G_{\Phi}^{-\infty}(X ; E)$ be given. Then there exists a neighborhood $\mathcal{U}$ of $z$ such that $\mathcal{U}$ has a non-empty intersection with only finitely many open sets of the covering (7.14), say $B_{\epsilon_{i_{1}}}\left(A_{i_{1}}\right), \ldots, B_{\epsilon_{i_{m}}}\left(A_{i_{m}}\right)$. Without loss of generality, assume that $B_{\epsilon_{i_{1}}}\left(A_{i_{1}}\right), \ldots, B_{\epsilon_{i_{m}}}\left(A_{i_{n}}\right), n \leq m$ are the open sets of the covering containing $z$, and assume that $\epsilon_{i_{1}}=\max \left\{\epsilon_{i_{1}}, \ldots, \epsilon_{i_{n}}\right\}$. By construction, we have

$$
z \in B_{\epsilon_{i_{k}}}\left(A_{i_{k}}\right) \subset B_{3 \epsilon_{i_{1}}}\left(A_{i_{1}}\right), \quad \forall k \in\{1, \ldots, n\},
$$

which implies that $\xi_{t}(z) \in B_{3 \epsilon_{i_{1}}}\left(A_{i_{1}}\right) \subset G_{\Phi}^{-\infty}(X ; E)$ for all $t \in[0,1]$.

Let $N$ be the nerve of the covering (7.14). Call $b_{i}$ the vertex that corresponds to the open set $B_{\epsilon_{i}}\left(A_{i}\right)$. Then $N$ is a CW-complex with affine simplices as cells. There is an obvious inclusion $\rho: N \rightarrow G_{\Phi}^{-\infty}(X ; E)$ given by sending the vertex $b_{i}$ to $A_{i}$ for $i \in \mathbb{N}$ and so that on any simplex of $N, \rho$ is an affine map. Since $\xi_{1}\left(G_{\Phi}^{-\infty}(X ; E)\right) \subset$ $N$, the homotopy (7.24) shows that the inclusion $\rho$ is a homotopy equivalence. By Whitehead's theorem, we then conclude that $G_{\Phi}^{-\infty}(X ; E)$ is contractible.

Not only is theorem 7.19 a generalization of the contractibility result of [18], it is also obtained using quite different methods. In [18], the proof of the contractibility relies on the fact that all the homotopy groups of the long exact sequence (7.2) are either trivial or isomorphic to $\mathbb{Z}$, which is not true in the general fibred cusp setting. It also uses explicit isomorphisms of the homotopy groups with $\mathbb{Z}$ given by the integration of various differential forms. Essentially, the proof is a computation which is showing surprising relations among these differential forms, almost as if it was an accident. Our proof is certainly longer and more elaborate, but it has the advantage of making the final result looks intuitively more natural. In fact, it seems to us that the proof in [18] would have been intuitively more natural written in the reverse way, that is, if one would have reduced the relations among the differential forms from the contractibility instead of the other way around. In this state of mind, let us reprove proposition 3.1 and 3.2 in [18] using the contractibility result of corollory 7.21 .

Proposition 7.24. Assume that $\Phi: \partial X \rightarrow\{\mathrm{pt}\}$ is the trivial fibration. Then for 
$k \in \mathbb{N}$, the pullback and integration of the differential forms

$$
\beta_{k}^{\text {odd }}=c_{k}^{\text {odd }} \operatorname{Tr}\left(\left(a^{-1} d a\right)^{2 k-1}\right), \quad c_{k}^{\text {odd }}=\frac{1}{(2 \pi i)^{k}} \frac{(k-1) !}{(2 k-1) !}
$$

give an explicit isomorphism

$$
\pi_{2 k-1}\left(\dot{G}^{-\infty}(X ; E)\right) \rightarrow \mathbb{Z}
$$

Similarly, for $k \in \mathbb{N}_{0}$, the pullback and the integration of the differential forms

$$
\beta_{k}^{\text {even }}=c_{k}^{\text {even }} \int_{\mathbb{R}} \operatorname{Tr}\left(\left(a^{-1} d a\right)^{2 k} a^{-1} \frac{d a}{d s}\right) d s, \quad c_{k}^{\text {even }}=\frac{1}{(2 \pi i)^{k+1}} \frac{k !}{(2 k) !}=\frac{c_{k}^{\text {odd }}}{4 \pi i}
$$

give an explicit isomorphism

$$
\pi_{2 k}\left(G_{\Phi s}^{-\infty}(\partial X ; E)\right) \rightarrow \mathbb{Z}
$$

Proof. Recall that $\dot{G}^{-\infty}(X ; E)$ and $G_{\Phi s}^{-\infty}(\partial X ; E)$ are classifying spaces for odd and even $K$-theory respectively. As in [18], the difficulty is to find the good values for the constants $c_{k}^{\text {odd }}$ and $c_{k}^{\text {even }}$. We will find them by induction on $k \in \mathbb{N}$. Recall that $c_{1}^{\text {odd }}=\frac{1}{2 \pi i}$ from standard winding number computations ${ }^{4}$ in $\operatorname{GL}(1, \mathbb{C}) \cong \mathbb{C}^{*}$. Moreover, from the computation (3.9) [18],

$$
c_{k}^{\text {even }}=(2 k+1) c_{k+1}^{\text {odd }}, \quad k \in \mathbb{N}_{0} .
$$

From the contractibility of $G_{\Phi}^{-\infty}(X ; E)$ and the computation (3.13) in [18], one has that

$$
c_{k}^{\text {even }}=\frac{c_{k}^{\text {odd }}}{4 \pi i} .
$$

This allows us to compute $c_{k}^{\text {even }}$ and $c_{k}^{\text {odd }}$ recursively.

Remark 7.25. Proposition 7.24 is a standard result about classifying spaces of $K$ theory. It is interesting to get the constant $c_{k}^{\text {odd }}$ and $c_{k}^{\text {even }}$ as a corollary of theorem 7.19. However, for someone only interested in those constants, this way of obtaining them is probably not worthwhile.

\subsection{The case $Y=\mathbb{S}^{1}$}

In the case $Y=\mathbb{S}^{1}$, we can also explicitly compute the homotopy groups of $G_{\Phi}^{-\infty}$.

Corollary 7.26. When $Y=\mathbb{S}^{1}$ and $\operatorname{dim} Z>0$, the homotopy groups of $G_{\Phi}^{-\infty}(X ; E)$ are given by

$$
\pi_{k}\left(G_{\Phi}^{-\infty}(X ; E)\right) \cong\left\{\begin{array}{cl}
\{0\} & k \text { even } \\
\mathbb{Z} & k \text { odd }
\end{array}\right.
$$

\footnotetext{
${ }^{4}$ That is, using $d \log a=a^{-1} d a$.
} 
Proof. In that case, the one point compactification of $T^{*} Y \times \mathbb{R}$ is homeomorphic to $\mathbb{S}^{3} / \mathbb{S}^{1}$. Thus, since

$$
\widetilde{K}^{i}\left(\mathbb{S}^{3}\right) \cong \widetilde{K}^{i}\left(\mathbb{S}^{1}\right) \cong\left\{\begin{array}{cl}
\{0\} & i=0 \\
\mathbb{Z} & i=1,
\end{array}\right.
$$

and using the fact that the homomorphism

$$
i^{*}: \widetilde{K}^{1}\left(\mathbb{S}^{3}\right) \rightarrow \widetilde{K}^{1}\left(\mathbb{S}^{1}\right)
$$

induced by the inclusion $i: \mathbb{S}^{1} \rightarrow \mathbb{S}^{3}$ maps everything to zero, we deduce from the six terms exact sequence

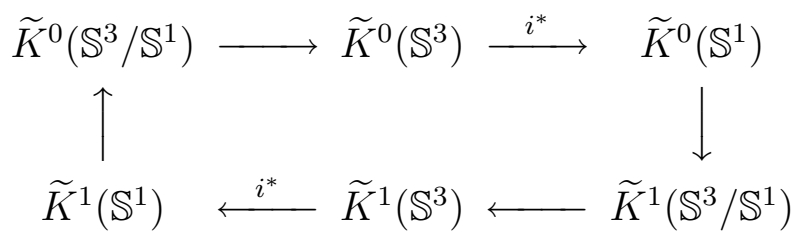

that

$$
K_{c}^{0}\left(T^{*} Y\right) \cong \widetilde{K}^{1}\left(\mathbb{S}^{3} / \mathbb{S}^{1}\right) \cong \mathbb{Z}, \quad \widetilde{K}^{1}\left(Y^{T^{*} Y}\right) \cong \widetilde{K}^{0}\left(\mathbb{S}^{3} / \mathbb{S}^{1}\right) \cong \mathbb{Z}
$$

Hence the result follows from theorem 7.19 together with the fact that the topological index

$$
\operatorname{ind}_{t}: K_{c}^{0}\left(T^{*} \mathbb{S}^{1}\right) \rightarrow \mathbb{Z}
$$

is an isomorphism (cf. remark 6.11). 


\section{Bibliography}

[1] M.F. Atiyah. K-theory. Benjamin, 1967.

[2] M.F. Atiyah and I.M. Singer. The index of elliptic operators: I. Ann. of Math., $87: 484-530,1968$.

[3] M.F. Atiyah and I.M. Singer. The index of elliptic operators: III. Ann. of Math., 87:546-604, 1968.

[4] R. Bott. The stable homotopy of the classical groups. Ann. of Math., 70:313-337, 1959.

[5] R. Bott and L.W. Tu. Differential forms in algebraic topology. Number 82. Springer-Verlag, Berlin, 1982.

[6] B. Hartley and T.O. Hawkes. Rings, modules and linear algebra. Chapman and Hall, London, 1970.

[7] L. Hörmander. The Analysis of Linear Partial Differential Operators. Vol. 3. Springer-Verlag, Berlin, 1985.

[8] N.H. Kuiper. The homotopy type of the unitary group of Hilbert space. Topology, 3:19-30, 1964.

[9] R. Lauter and S.Moroianu. An index formula on manifolds with fibered cusp ends. to appear in J. Geom. Analysis.

[10] R. Lauter and S.Moroianu. Fredholm theory for degenerate pseudodifferential operators on manifold with fibred boundaries. Comm. Partial Differential Equations, 26:233-283, 2001.

[11] R. Lauter and S.Moroianu. Homology of pseudodifferential operators on manifolds with fibered cusps. T. Am. Soc., 355:3009-3046, 2003.

[12] R. Mazzeo and R. B. Melrose. Pseudodifferential operators on manifolds with fibred boundaries. Asian J. Math., 2(4):833-866, 1999.

[13] R.B. Melrose. Analysis on manifolds with corners. In preparation.

[14] R.B. Melrose. The Atiyah-Patodi-Singer index theorem. A. K. Peters, Wellesley, Massachusetts, 1993. 
[15] R.B. Melrose. The eta invariant and families of pseudodifferential operators. Math. Res. Lett., 2(5):541-561, 1995.

[16] R.B. Melrose. Geometric Scattering theory. Cambridge University Press, Cambridge, 1995.

[17] R.B. Melrose and P. Piazza. Families of Dirac operators, boundaries and the b-calculus. J. Differential Geom., 46(1):99-180, 1997.

[18] R.B. Melrose and F. Rochon. Families index for pseudodifferential operators on manifolds with boundary. IMRN, 22:1115-1141, 2004.

[19] H. Shubert. Topology. Allyn and Bacon Inc, Boston, 1968.

[20] M.A. Shubin. Pseudodifferential operators and spectral theory. Springer-Verlag, Berlin-Heidelberg-New York, 1987. (Nauka, Moscow, 1978).

[21] N. Steenrod. The topology of fibre bundles. Princeton University Press, New Jersey, 1999. 